\title{
Understanding and treating obesity in daily life
}

Citation for published version (APA):

Boh, B. (2017). Understanding and treating obesity in daily life: a cognitive behavioral perspective. [Doctoral Thesis, Maastricht University]. Maastricht University. https://doi.org/10.26481/dis.20170413bb

Document status and date:

Published: 01/01/2017

DOI:

10.26481/dis.20170413bb

Document Version:

Publisher's PDF, also known as Version of record

Document license:

Unspecified

\section{Please check the document version of this publication:}

- A submitted manuscript is the version of the article upon submission and before peer-review. There can be important differences between the submitted version and the official published version of record.

People interested in the research are advised to contact the author for the final version of the publication, or visit the DOI to the publisher's website.

- The final author version and the galley proof are versions of the publication after peer review.

- The final published version features the final layout of the paper including the volume, issue and page numbers.

Link to publication

\footnotetext{
General rights rights.

- You may freely distribute the URL identifying the publication in the public portal. please follow below link for the End User Agreement:

www.umlib.nl/taverne-license

Take down policy

If you believe that this document breaches copyright please contact us at:

repository@maastrichtuniversity.nl

providing details and we will investigate your claim.
}

Copyright and moral rights for the publications made accessible in the public portal are retained by the authors and/or other copyright owners and it is a condition of accessing publications that users recognise and abide by the legal requirements associated with these

- Users may download and print one copy of any publication from the public portal for the purpose of private study or research.

- You may not further distribute the material or use it for any profit-making activity or commercial gain

If the publication is distributed under the terms of Article $25 \mathrm{fa}$ of the Dutch Copyright Act, indicated by the "Taverne" license above, 
Bastiaan Boh

\section{Understanding and}

\section{treating obesity}

in daily life

A cognitive behavioral

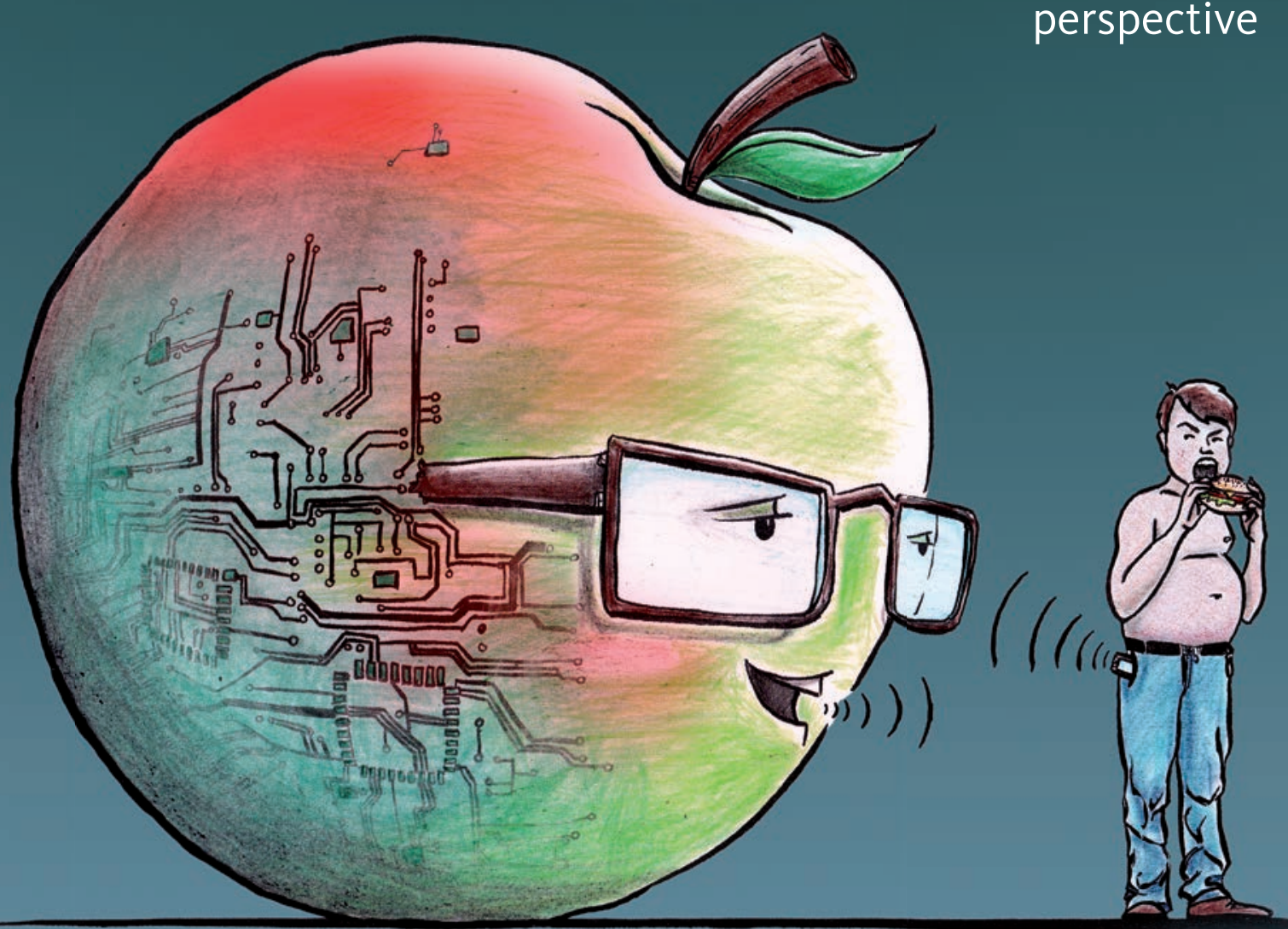



Understanding and treating obesity in daily life A cognitive behavioral perspective

Bastiaan Boh 
(C) copyright Bastiaan Boh, Maastricht 2017

Cover design: Nynke Dijkstra.

Layout: Ridderprint BV - www.ridderprint.nl

Printed by: Ridderprint BV - www.ridderprint.nl 


\section{Understanding and treating obesity in daily life A cognitive behavioral perspective}

\section{PROEFSCHRIFT}

Ter verkrijging van de graad van doctor aan de Universiteit Maastricht, op gezag van de Rector Magnificus, Prof. Dr. Rianne Letschert, volgens het besluit van het College van Decanen,

in het openbaar te verdedigen op donderdag 13 april 2017 om 12:00 uur

door

Bastiaan Boh 


\section{Promotor}

Prof. Dr. A. Jansen

\section{Copromotoren}

Dr. A. Roefs

Dr. L. H. J. M. Lemmens

Dr. C. Nederkoorn

\section{Beoordelingscommissie}

Prof. Dr. G. J. Kok (voorzitter)

Dr. M. van Boxtel

Prof. Dr. S. Kremers

Prof. Dr. H. Riper (Vrije Universiteit Amsterdam)

Prof. Dr. P. Spinhoven (Universiteit Leiden)

The research presented in this dissertation was funded by grant 12028 from Stichting Technische Wetenschappen (STW), Nationaal Initiatief Hersenen en Cognitie (NIHC), Nederlandse Organisatie voor Wetenschappelijk Onderzoek (NWO) and Philips under the Partnership programme Healthy Lifestyle Solutions. The studies were conducted at Maastricht University. 


\section{CONTENTS}

Chapter 1 General introduction 7

\section{PART 1 ECOLOGICAL MOMENTARY ASSESSMENT OF EATING- RELATED COGNITIONS, EMOTIONS, FOOD DESIRE AND FOOD INTAKE}

Chapter 2 Indulgent thinking? Ecological Momentary Assessment of

Overweight and Healthy-weight Participants' Cognitions and Emotions

Chapter 3 Food Desire in Daily Life: Comparison of overweight and healthyweight participants via ecological momentary assessment

\section{PART 2 COGNITIVE BEHAVIORAL THERAPY-BASED ECOLOGICAL MOMENTARY INTERVENTION FOR OBESITY}

Chapter 4 An ecological momentary intervention for weight loss and healthy eating via smartphone and internet: Study protocol for a randomised controlled trial

Chapter 5 Randomized Controlled Trial of a Cognitive Behavioural Therapybased Ecological Momentary Intervention for Obesity

\section{Chapter 6}

General Discussion

Summary (English)

Summary (Dutch)

Valorization addendum

Acknowledgments

Reference list 

Chapter 1

General Introduction 

Obesity has reached pandemic proportions (Flegal, Carroll, Kit, \& Ogden, 2012; Flegal, Carroll, Ogden, \& Curtin, 2010; Swinburn et al., 2011). Between 1980 and 2013, the world-wide prevalence of overweight has risen from $29 \%$ to $37 \%$ ( $\mathrm{Ng}$ et al., 2014). It is associated with a large healthcare burden (Cawley \& Meyerhoefer, 2012; Dixon, 2010; McKinsey Global Institute, 2014), which is still increasing (Wang, McPherson, Marsh, Gortmaker, \& Brown, 2011). In addition, obese individuals are more likely to suffer from social stigma (Puhl \& Heuer, 2010), experience a reduced quality of life (Jia \& Lubetkin, 2005), and are more likely to be depressed than non-obese individuals (Scott et al., 2007).

As a result, increasingly more people are worried about their weight. Already in 2001 and $2002,48 \%$ of women and $34 \%$ of men in the U.S. reported trying to lose weight (Weiss, Galuska, Khan, \& Serdula, 2006). In 2014, around 63\% of the general Dutch population qualified as a dieter (De Ridder, Adriaanse, Evers, \& Verhoeven, 2014). It appears that concerns about health and excess weight are pervasive in the general population, even for those who are not overweight (Rozin, Fischler, Imada, Sarubin, \& Wrzesniewski, 1999). However, most dieting attempts are not successful in the long term (for a review, see Franz et al., 2007).

In the present thesis, a treatment method for weight loss and healthier eating is investigated, which makes use of recent technological innovations. That is, an e-coach is developed that delivers treatment in the context of daily life (Atkinson, Gold, \& Ballard, 2002). The treatment is based on cognitive behaviour therapy (CBT) for weight loss (Beck, 2008; Werrij, 2005), which teaches clients to deal with hunger, food desires, and temptations to eat, trains clients how to identify and evaluate dysfunctional cognitions (thoughts) that promote overeating, and how to replace such cognitions with functional alternative cognitions. In addition to developing and testing the e-coach, two observational studies are included, which focus on (1) eating-related cognitions and emotions, and (2) food desires, in the context of daily life. To illustrate why an e-coach for weight loss might be useful, the next sections will focus on existing weight loss treatments and their limitations, as well as introduce the concepts of ecological momentary assessment (EMA) and ecological momentary intervention (EMI).

\section{EFFECTIVE WEIGHT LOSS TREATMENTS}

When thinking about weight loss, a common conception is that it is a matter of maintaining a negative energy-balance (i.e., when the human body burns more calories than it gains). The inevitable consequence is weight loss. Although this conception is correct (Speakman \& Mitchell, 2011), it does not convey why weight loss is so difficult. Effectively restraining food intake (i.e., dieting) is a process requiring deliberate thought (Herman \& Mack, 1975), high self-efficacy (McKee, Ntoumanis, \& Taylor, 2014), and self-control (Abramson, 1977; Cohen, Gelfand, Dodd, Jensen, \& Turner, 1980; Hofmann, Friese, \& Roefs, 2009). 
Self-control is complicated by an 'obesogenic' environment, which is replete with food temptation (Egger \& Swinburn, 1997; Swinburn et al., 2011). High-caloric foods are increasingly more abundant and easy to obtain and consume (Mozaffarian et al., 2011). This is a problematic development because high-caloric foods also elicit food desires and craving (Pelchat, 2002). Self-control is also complicated by psychological processes (Jansen, Houben \& Roefs, 2015). For example, obese individuals are found to be more sensitive to the rewarding properties of high-caloric foods than healthy-weight individuals (Davis, Strachan, \& Berkson, 2004), in spite of negative consequences, such as weight gain. It is no surprise, then, that obese individuals also report more frequent high-caloric food desires than healthy-weight individuals (Chao, Grilo, White, \& Sinha, 2014; Delahanty, Meigs, Hayden, Williamson, \& Nathan, 2002; Franken \& Muris, 2005; Ouwehand \& Papies, 2010).

So far, efforts to achieve effective weight loss have been largely ineffective (e.g., Brownell, 2010). Behavioral treatment programs (Butryn, Webb \& Wadden, 2011), which focus on setting goals (e.g., for weight loss), and stimulus control (e.g., changing elements in the environment that are associated with high-caloric food intake), often result in modest initial weight losses of around 8-10\% (Butryn, Webb, \& Wadden, 2011). Such losses are clinically meaningful, as weight loss of around $5-10 \%$ is associated with health improvement (Goldstein, 1992; Mertens \& Van Gaal, 2000). However, these modest results are commonly not in line with people's expectations (Cooper \& Fairburn, 2001) and, most importantly, are difficult to maintain. Meta-analyses show that diet programs (Anderson, Konz, Frederich, \& Wood, 2001; Atallah et al., 2014; Franz et al., 2007; Johnston et al., 2014), very low-calorie diets (Franz et al., 2007), lifestyle programs (Douketis, Macie, Thabane, \& Williamson, 2005; Franz, Boucher, Rutten-Ramos, \& VanWormer, 2015), pharmacotherapy (Douketis et al., 2005), and dietetic counseling (Dansinger, Tatsioni, Wong, Chung, \& Balk, 2007) generally do not result in clinically significant long-term weight loss, as most or all of the weight is almost invariably regained within five years (Anderson et al., 2001; Franz et al., 2007; Jeffery et al., 2000). In addition, more intensive therapist-guided behavior therapy programs also show a pattern of weight regain after weight loss (Shaw, Rourke, Del Mar, \& Kenardy, 2005). In fact, in general, only a small percentage of dieters, between 3\% (Anderson et al., 2001; Franz et al., 2007; Wing \& Hill, 2001) and 21\% (Wing \& Hill, 2001; Wing \& Phelan, 2005), depending on the definition of success, manages to keep the weight off in the long term (Ikeda et al., 2005). An exception to this is the weight loss and weight loss maintenance that can be achieved with bariatric surgery. Although bariatric surgery is associated with health risks such as surgical complications and even death, it also allows patients to achieve more effective long-term weight loss (Chang et al., 2014).

Obese individuals often get caught up in trying to optimize the macronutrient composition of their diet, as is evident from the blooming diet industry. However, finding the perfect diet may not be essential for effective weight loss. It was found that diet adherence is a better predictor of weight loss than the macronutrient composition of a diet (Johnston 
et al., 2014; Sacks et al., 2009). One reason for this is that many obese individuals become chronic dieters and continuously alternate between periods of successful weight loss, and periods of failure to self-regulate their behavior in the face of food temptation (Hofmann, van Koningsbruggen, Stroebe, Ramanathan, \& Aarts, 2010; Papies, Stroebe, \& Aarts, 2009). When confronted with dietary temptations, such ineffective dieters will not know how to deal with them, and proceed to (over)eat (Carels et al., 2001; Carels, Douglass, Cacciapaglia, \& O'Brien, 2004; McKee et al., 2014). So, rather than emphasize the type of diet that is picked, it may be more effective to target the (psychological) processes that cause obese individuals to give in to dietary temptations.

\section{COGNITIVE BEHAVIORAL THERAPY FOR WEIGHT LOSS}

Eating-related cues such as the sight and smell of food, or the associations of certain environments (e.g., a restaurant) with food intake, should not necessarily be considered dietary temptations. In comparable obesogenic environments, not everyone becomes overweight, which implies that there are differences in how susceptible people are to the influence of eating-related cues and associations. Instead, the perception and the interpretation of cues and associations, through accompanying thoughts and emotions, can cause people to be tempted to eat. Therefore, such eating-related thoughts and emotions might be suitable targets for obesity treatment (Karhunen et al., 2012). In line with this idea, it has been proposed that dysfunctional thoughts (cognitions) regarding eating (e.g., "If I refuse food offered to me at a party, everyone will think that I am rude") may be important determinants of problematic eating behaviour (Carter \& Jansen, 2012; Karhunen et al., 2012; O'Connor \& Dowrick, 1987). Having a dichotomous thinking style (e.g., "My diet is either a success or a failure") was found to be one of the main determinants for weight regain (Byrne, Cooper, \& Fairburn, 2004). In addition, obesity was found to be associated with an increased presence of thoughts that condone giving in to dietary temptation (O'Connor \& Dowrick, 1987; Tiggemann, 2000), and with concerns about weight and shape (Nauta, Hospers, Jansen, \& Kok, 2000).

Cognitions are central to cognitive behavioral therapy (CBT; Beck, 2011). CBT is one of the most common psychological treatments worldwide (Hollon \& Beck, 1994), and is used to treat a wide variety of disorders, including eating disorders (Fairburn, Cooper, \& Shafran, 2003). In essence, the theory behind CBT states that cognitions and beliefs can lead to a negative mood and undesirable behavior, such as overeating (Beck, 2008; 2011; Jansen, Mulkens \& Jansen, 2011; Jansen \& Mulkens, 2015). Such dysfunctional cognitions (cognitive distortions) are usually based on errors in reasoning and inference (such as over-generalization). They are commonly formulated in an if-then format, such as "If food is offered to me, then refusing it is impolite", or "if I do not lose weight, I will never look good". 


\section{Cognitions, emotions and obesity}

For obesity, dysfunctional cognitions occur in various ways. They can occur when situations or circumstances give rise to a temptation to eat, such as being in the presence of others who are eating ("If everyone's eating cake, I should join them"), or when encountering cues that are associated with eating (e.g., food advertisements that emphasize the rewarding properties of high-caloric food; Beck, 2008). Such cognitions can sometimes be ambiguous and only dysfunctional if they occur frequently (e.g., "One snack is not going to hurt my diet $^{\prime \prime}$ ). Dysfunctional cognitions can also be related to the consequences of overeating. Such cognitions occur when failing to adhere to a diet (i.e., by giving in to dietary temptations; e.g., "if I fail my diet, it must mean I am a weak person"), and can be about weight and shape (e.g., "If I can't wear normal-sized clothes, I am worthless"). In line with this, it was found that obese participants reported more exercise and diet cognitions after social comparisons (Leahey, Crowther, \& Ciesla, 2011; Rancourt, Leahey, Larose, \& Crowther, 2015). Also, related to obesity, participants scoring high on binge eating disorder symptomatology reported more negative emotion cognitions before and after a binge eating event and loss of control (Goldschmidt, Crosby, Cao, et al., 2014; Goldschmidt, Crosby, Engel, et al., 2014).

There is also evidence that emotions, which also play a central role in CBT (Beck, 2008; 2011), influence eating behavior. The occurrence of negative emotions was predictive of high-caloric food intake, both in obese and non-obese individuals (Cardi, Leppanen, \& Treasure, 2015). Observational studies so far have found that states of affective arousal, in particular the experience of positive (Carels et al., 2004; Tomiyama, Mann, \& Comer, 2009) and negative emotions (Carels et al., 2001; Kubiak, Vögele, Siering, Schiel, \& Weber, 2008), were predictive of temptations and dietary lapses in both obese and non-obese dieters. Minor daily stressors also predicted high-caloric food desires in adolescents (Kubiak, Vögele, Siering, Schiel, \& Weber, 2008). Anxiety, however, did not predict lapses in a non-obese sample of dieters (Tomiyama et al., 2009). In short, findings so far have indicated the influence of many different emotions on eating behavior.

\section{CBT for obesity: model and treatment}

The influence of cognitions and emotions on eating behavior can be synthesized in a CBT model for obesity. Such a CBT model for obesity postulates that eating-related dysfunctional cognitions and emotions obstruct dieting efforts and perpetuate overeating behavior (Beck, 2008; Werrij et al., 2009). This implies a vicious cycle, as overeating leads to more dysfunctional cognitions about eating, dieting, shape and weight, such as "If I fail to resist food temptations, then I might as well continue eating", and may increase belief in a negative selfimage, leading to reduced self-efficacy (Meijboom, Jansen, Kampman \& Schouten, 1999). Such cognitions, in turn, reinforce negative emotions, and make consecutive overeating more likely. So, to achieve effective weight loss, this vicious cycle has to be broken. 
CBT for obesity teaches clients to identify problematic eating behaviors, and the dysfunctional cognitions that are associated with such behaviors, through self-monitoring and therapeutic techniques: by changing eating-related dysfunctional cognitions, problematic behavior is consequently also changed (Beck, 2008; 2011; Cooper et al., 2010; Gade, Hjelmesæth, Rosenvinge, \& Friborg, 2014; Stahre \& Hällström, 2005; Stahre, Tärnell, Håkanson, \& Hällström, 2007; Werrij et al., 2009). First, identified dysfunctional cognitions are evaluated in terms of how believable they are, and are gradually, over the course of treatment devalued. This is, for example, done by asking participants open-ended, self-guiding (i.e., Socratic) questions (e.g., "What is the evidence in favor of, or against this thought?"), and by conducting behavioral experiments to investigate the validity of their thoughts (Beck, 2011; Overholser, 2010). Then, clients learn to formulate functional alternative cognitions, which gradually become more believable and replace the devalued dysfunctional cognitions. Examples of other common CBT techniques through which dysfunctional cognitions are gradually devalued and replaced by functional alternative cognitions are: exposure to food temptation, but not being allowed to eat, setting up and conducting behavioral experiments in which dysfunctional beliefs are put to the test, and self-monitoring (e.g., by keeping food diaries, in which eating-related thoughts and emotions, food desire and intake, and reasons for consumption are tracked). Aside of dysfunctional cognitions, CBT for obesity commonly also targets self-esteem, self-confidence and body-image, and participants are prepared for the post-treatment weight loss maintenance phase.

Research has shown that CBT for obesity was able to achieve moderate weight loss (between approximately 4\% and 9\%) that was maintained at a 1-year follow-up measurement in some trials (Sbrocco, Nedegaard, Stone, \& Lewis, 1999; Stahre \& Hällström, 2005; Stahre, Tärnell, Håkanson, \& Hällström, 2007; Werrij et al., 2009). However, not all trials have resulted in successful weight loss maintenance. In Cooper et al. (2010), participants in all three conditions (CBT, behavior therapy, and a general lifestyle program) lost comparable significant weight (between approximately $5 \%$ and $10 \%$ ) in the acute phase, but they had regained all of the weight at the subsequent three-year follow-up measurement.

Considering the mixed findings that CBT trials for obesity present, there is a need for further insight into how CBT for obesity can be improved. In line with this, it is suggested that it may be important to help obese individuals deal with dietary temptations and food desires as they occur (Carels et al., 2001; Carels et al., 2004; Jansen et al., 2011; Mckee et al., 2014), so CBT could benefit from treatment provided directly in people's daily lives. However, this is not feasible in a traditional treatment session setting. Also, as obesity is sometimes considered a chronic condition (Bray, 2004; Rippe, Crossley, \& Ringer, 1998), and as it is difficult to attain long-term weight loss maintenance (e.g., Franz et al., 2007), extending treatment duration may lead to more effective weight loss maintenance (Middleton, Patidar, \& Perri, 2012). So, the effects of providing potentially indefinite CBT for obesity in obese individuals' daily lives is one way by which treatment effectiveness may be increased. 


\section{From the clinic to the overeating environment: treatment in daily life}

One way to account for individual circumstances that give rise to dietary temptations, and to overcome practical treatment issues, is to provide treatment via the internet or mobile technology (e-Health; Griffiths et al., 2006; Ramadas, Quek, Chan \& Oldenburg, 2011), a method which is becoming increasingly popular (Broekhuizen, Kroeze, Poppel, Oenema \& Brug, 2012). Internet treatment has the advantage of being relatively cost effective (Gerhards et al., 2010), and can reduce therapist time, or require no therapist involvement at all, which reduces waiting lists (Foroushani, Schneider, \& Assareh, 2011). Evidence from several meta-analyses on effective internet treatment for weight loss maintenance is mixed. Some evidence suggests that behavioral internet treatment for weight loss is as effective as face-to-face treatment for promoting long-term weight loss maintenance of at least $5 \%$ of the original weight (Harvey-Berino, Pintauro, Buzzell, \& Gold, 2004; Tate, Jackvony, \& Wing, 2006; van Wier et al., 2009). However, it was also found that personalized feedback-based internet-delivered behavioural therapy for obesity no longer differed from non-personalized control conditions in terms of maintained weight loss at one-year follow-up measurements (Sherrington et al., 2016; van Wier et al., 2009). Effective internet interventions include elements of (cognitive) behaviour therapy, such as self-monitoring of (eating) behaviour, and individually tailored (personalized) feedback through progress-related charts and based on diary entries, which could either be automated or facilitated (Krukowski, Harvey-Berino, Ashikaga, Thomas, \& Micco, 2008; Saperstein et al., 2007; Sherrington et al., 2016; Tate et al., 2001). Also, adding progress tracking methods to an internet weight loss website was found to be beneficial for weight loss, whereas adding social support elements was found to be beneficial for weight loss maintenance (Krukowski et al., 2008).

CBT is also being provided increasingly more often via the internet and mobile platforms. However, such interventions mostly do not directly target obesity or weight loss. For example, in a sample of obese adolescent binge eaters, computerized CBT (CCBT) was moderately effective at achieving weight stabilization, and highly effective at reducing binge eating, compared to a wait-list control condition (Jones et al., 2008). cCBT was also used to effectively treat disorders such as insomnia (Vincent, Walsh, \& Lewycky, 2010), generalized anxiety disorder (Amir \& Taylor, 2012; Andrews, Cuijpers, Craske, McEvoy, \& Titov, 2010), and depression (Andrews et al., 2010; Cooper et al., 2011; de Graaf et al., 2011; Foroushani et al., 2011; Kaltenthaler, Parry, Beverley, \& Ferriter, 2008).

\section{EATING BEHAVIOR AND WEIGHT LOSS TREATMENT IN DAILY LIFE}

To enable CBT via the internet and mobile platforms, it is first necessary to obtain insights into clients' specific problematic eating behavior. To do this, ecological momentary assessment (EMA) is used in the present thesis, conducted via an iPhone app (Chapters 2 and 3). 
Essentially, EMA is the repeated assessment of aspects of daily life that are of interest to a researcher (Shiffman, Stone, \& Hufford, 2008). It allows more micro-level insights of factors such as emotions, contexts, and cognitions that are related to behavior of interest. Instead of one cross-sectional investigation, many repeated observations are obtained. EMA can easily be implemented on a smartphone, by sending signals to the phone when participants are required to complete a brief questionnaire about eating-related factors, or by instructing participants to start an assessment prior to a relevant event, such as food intake. EMA has the added advantage of avoiding memory biases that are associated with retrospective questionnaire assessment (Schwarz, 2007). The method has previously been used to assess eating-related factors in daily life (Engel et al., 2016), and most EMA studies so far have focused on dieters and dietary temptations (Carels et al., 2001; Carels, Douglass, Cacciapaglia, \& O'Brien, 2004; Hofmann, Adriaanse, Vohs, \& Baumeister, 2013; McKee, Ntoumanis, \& Taylor, 2014).

Using EMA data, it is possible to intervene directly in daily life, based on participants' individual data patterns, a therapy delivery method which is called 'ecological momentary intervention' (EMI). EMI has been used to treat various conditions, including obesity (see Heron \& Smyth, 2010 for an overview). Treatment can be provided promptly by, for example, warning participants about risky situations in which they may experience food desires (Heron \& Smyth, 2010), or by compiling useful feedback overviews (graphs and charts) from EMA data (Kramer et al., 2014). For weight loss treatment, results so far have been mixed, with some studies resulting in weight loss (Agras, Taylor, Feldman, Losch, \& Burnett, 1990; Burnett et al., 1985; Patrick et al., 2009), but only one study resulting in significant weight loss maintenance at eight months post-intervention (Burnett et al., 1985). Also, one EMI study did not find any significant weight loss post-intervention (Shapiro et al., 2012). Aside of weight loss, EMls have been used to effectively promote healthy food intake (Atienza, King, Oliveira, Ahn, \& Gardner, 2008; Nour, Chen, \& Allman-Farinelli, 2015), and to prevent weight gain (e.g., stabilize weight) in young adults (Partridge et al., 2015). So, although some studies show promising results, more research is needed to optimize EMls for weight loss and eating behavior.

\section{OUTLINE AND HYPOTHESES}

The present thesis concerns the development and application of a mobile and internetbased CBT treatment for weight loss. First, associations between emotions, cognitions, food desires and food intake are investigated via EMA (Chapters 2 and 3). Then, the design of a self-learning e-coach (iPhone app) and internet program for treating obesity via a CBT model of obesity (Think Slim) is discussed (Chapter 4). Lastly, the Think Slim EMI is compared to a self-guided diet-only control condition with regards to weight loss, belief in (and 
frequency of) dysfunctional cognitions, eating styles, eating disorder psychopathology, general psychopathology, and self-esteem, in a randomized controlled trial (RCT; Chapter 5).

\section{EMA study into emotions, cognitions, food desires and food intake}

In the first (EMA) study, participants receive iPhone notifications at pseudorandom times throughout the day. After receiving a notification, participants complete a brief questionnaire addressing food desires, emotions, locations and activities. In addition, participants

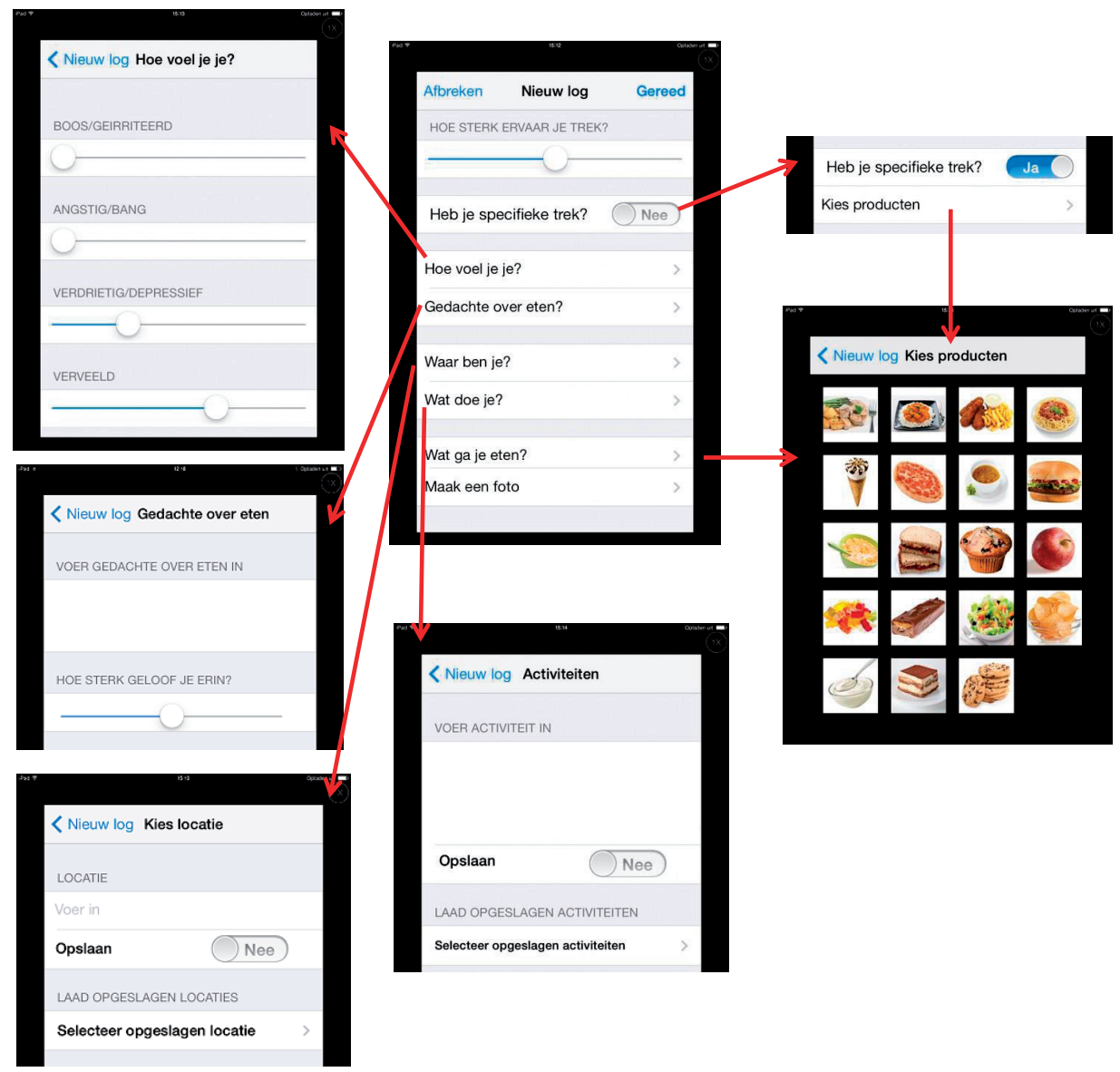

Figure 1. Assessing an eating event in the Think Slim EMA app.

Note. The app (in Dutch) assesses food desire, food desire specificity, emotions, cognitions, locations, activities, and food intake. Food desire is assessed via a VAS that defaults to the middle of the scale after participants start an assessment. Emotions are assessed using VAS that default at a score of zero. Cognitions, locations and activities are assessed via free text entries. For locations and activities, previous entries could be stored and recalled at subsequent assessments. The assessment ended with taking a picture of the food. For the present thesis, only the questions pertaining to food desire, food desire specificity, emotions, cognitions, and food intake are relevant. 
are instructed to complete questionnaires prior to eating events (Figure 1). At these eating events, the app also requests the assessment of cognitions and food intake. The data of this study are discussed in Chapters 2 and 3.

In Chapter 2, eating-related cognitions and emotions are compared between overweight and healthy-weight participants. The cognitive model for obesity predicts that obese participants should have more dysfunctional cognitions and negative emotions prior to high-caloric eating events (e.g., Beck, 2008; Werrij, 2005). Accordingly, it is hypothesized (1) that overweight participants have more dysfunctional cognitions than healthy-weight participants when about to eat high-caloric foods, and (2) that overweight participants have more negative emotions prior to high-caloric eating events than healthy-weight participants.

Additionally, influential previous work suggests negative emotions to play a causal role in high-caloric overeating in individuals who self-identify as so-called emotional eaters (as assessed by questionnaires, such as the Dutch Eating Behavior Questionnaire (van Strien, Frijters, Bergers, \& Defares, 1986), and the Emotional Eating Scale (Arnow, Kenardy, \& Agras, 1995). Recent, work, however, has suggested that these questionnaires measure people's beliefs about their eating behavior, rather than actual eating behavior (e.g., Adriaanse et al.,

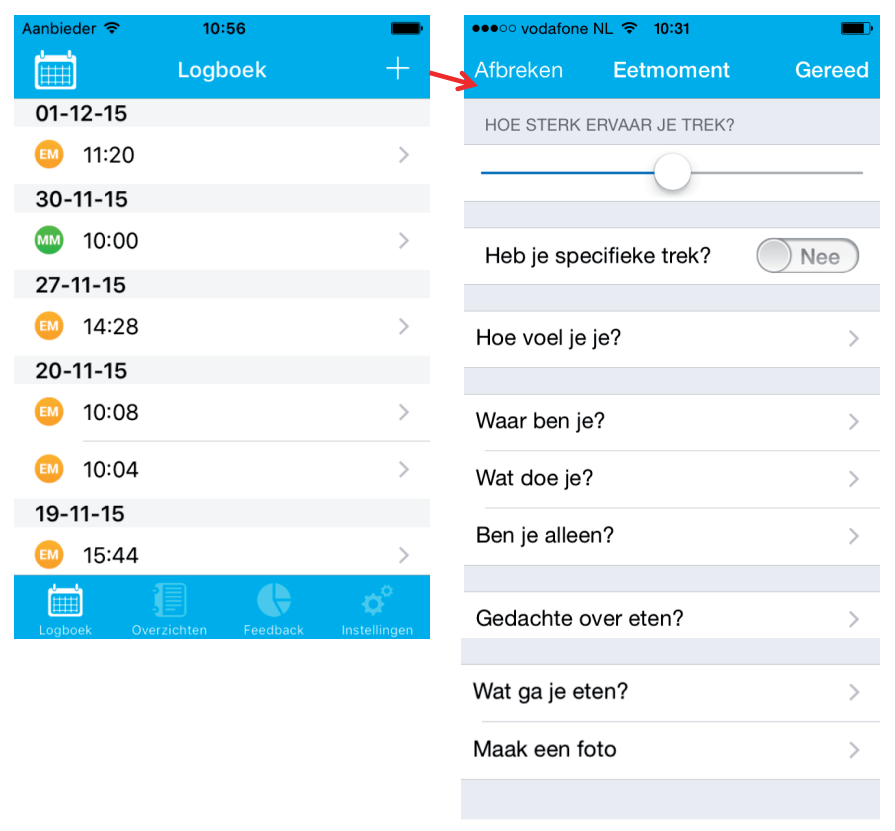

Figure 2. Logbook and eating event questionnaire of the Think Slim intervention app.

Note. The logbook (which was also present in the EMA-only app of the first study) contains an overview of all eating events and moments of dietary temptation that are assessed. 
2011; Bongers, de Graaff, \& Jansen, 2016; Evers et al., 2009). So, it is expected that positive associations between scores on emotional eating questionnaires and the occurrence of negative emotions or emotion-related cognitions prior to high-caloric eating events would be present at eating events and at non-eating moments.

Chapter 3 focuses on the EMA data for desire and food intake. Previous research showed that obese participants have more high-caloric food desires (Chao et al., 2014; Franken \& Muris, 2005). However, most studies investigating food desires have relied on retrospective self-reports or laboratory inductions, rather than ecologically valid observations. EMA studies on food desires are less common, but have so far focused mainly on food-related temptations and dietary lapses (Carels et al., 2001; McKee et al., 2014) and have not compared overweight and healthy-weight participants. Based on the previous comparisons between overweight and healthy-weight participants, it is hypothesized that overweight participants have more frequent and stronger food desires for high-caloric foods than healthy-weight

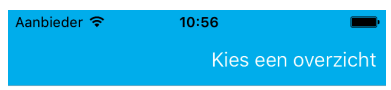

Wat doe ik als ik iets dat niet ongezond is eet?
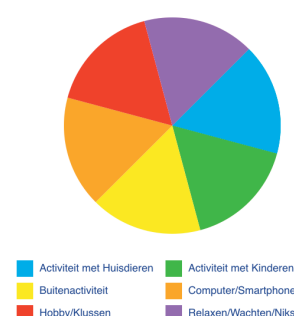

Hobby/KIussen

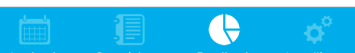

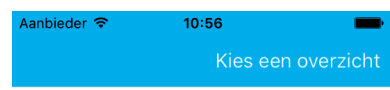

Waar ben ik als ik iets dat niet ongezond is eet?

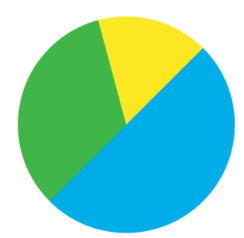

$$
\text { Buiten School }
$$

Thuis

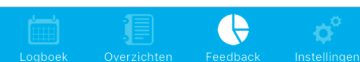

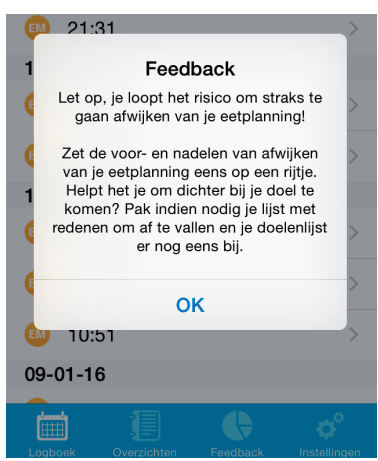




\begin{tabular}{|c|c|c|}
\hline 1 & \multicolumn{2}{|c|}{ Gedachte over eten categoriën } \\
\hline \multicolumn{3}{|c|}{$\begin{array}{l}\text { KIES DE CATEGORIE DIE HET BESTE PAST BIJ } \\
\text { JE GEDACHTE OVER ETEN }\end{array}$} \\
\hline (-) & Etenstijd & $>$ \\
\hline 6 & Energie & $>$ \\
\hline \%) & Gezonde intentie & $>$ \\
\hline 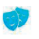 & Emotioneel & $>$ \\
\hline$\forall$ & Controle & $>$ \\
\hline 8 & Sociaal & $>$ \\
\hline 1 & Praktisch & $>$ \\
\hline 당 & Overig & $>$ \\
\hline
\end{tabular}

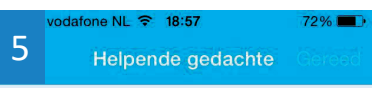

FORMULEER NU OP BASIS VAN JE ANTWOORDEN OP DE EVALUATIEVRAGEN EEN MEER HELPENDE/STEUNENDE GEDACHTE

\section{2}

\section{Gedachte over eten}

KIES DE GEDACHTE DIE JE HEBT OVER ETEN

ledereen neemt iets!

Het wordt van mij verwacht

Het is onbeleefd om nee te zeggen >

Ik wil niemand teleur stellen

$\begin{array}{rr}\text { vodafone NL } ₹ 18: 57 & 72 \% \\ \text { Gedachte over eten } & \text { Gereed }\end{array}$

JE GEDACHTE OVER ETEN

\section{ledereen neemt iets!}

HOE GELOOFWAARDIG IS DEZE GEDACHTE VOOR JOU?

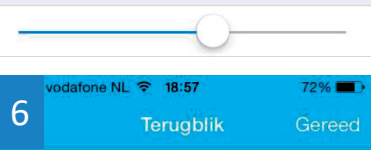

KIJK HIERONDER NOG EENS NAAR DE OORSPRONKELIJKE GEDACHTE

Als iedereen iets neemt, dan betekent dat dat ik dat ook moet doen

\section{HOE GELOOFWAARDIG IS DEZE GEDACHTE OP DIT MOMENT?}

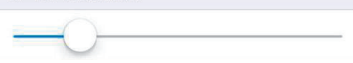

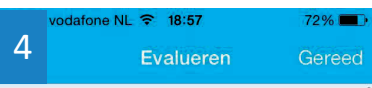

GEBRUIK ONDERSTAANDE

EVALUATIEVRAGEN OM JE GEDACHTE TE TOETSEN

\begin{tabular}{ll} 
Bewijzen voor en tegen & $>$ \\
\hline Maak ik een denkfout? & $>$ \\
\hline Alternatieve verklaring & $>$ \\
\hline Realistische conclusie & $>$ \\
\hline Helpt het mij? \\
\hline Voordelen van veranderen \\
\hline Advies aan vriend(in) \\
\hline Wat ga ik nu doen?
\end{tabular}

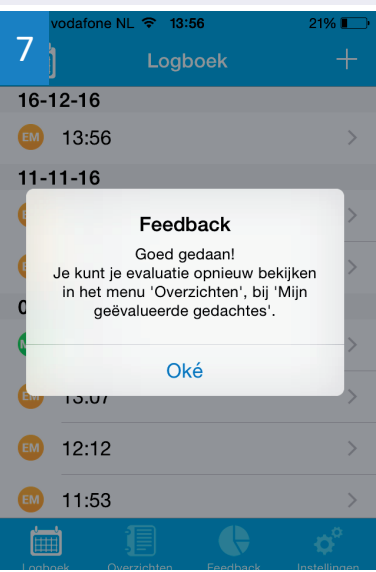

C

HOE GELOOFWAARDIG IS DEZE GEDACHTE VOOR JOU?

Figure 3. Methods of intervention in the Think Slim intervention app. Note.

A. Passive feedback charts are generated by the Think Slim intervention app, based on EMA data. The overviews depict the association between emotions and food desire, locations and high-caloric / other food intake, and activities and high-caloric / other food intake.

B. Adaptive feedback is based on an algorithm that estimates how likely it is that a participant will have a high-caloric food intake event in the subsequent time period (of, on average, 2 hours). After completing an EMA assessment in the app, the participant gets a warning message and a CBT-related instruction.

C. If the app detects that a cognition selected by a participant is dysfunctional, the participant is instructed to evaluate the cognition in the app, using standard CBT-related Socratic questions. The cognition is first selected (1 and 2), believability is scored (3), the cognition is evaluated (4), an alternative (functional) cognition is formulated (5), the original (dysfunctional) cognition is again scored on believability (6) and a feedback message is displayed (7). 
participants. In addition, based on previous work showing that overweight participants had more varied food intake (Raynor, Jeffery, Tate, \& Wing, 2004), it is hypothesized that this difference is also present for food desires, and that overweight participants therefore have more varied food desires and food intake than healthy-weight participants.

\section{RCT comparing a CBT-based ecological momentary intervention for obesity with a diet-only control}

For the second part of the thesis, an ecological momentary intervention (EMI), called Think Slim, is created, which uses an improved and expanded version of the app of the first EMA study. The Think Slim EMI consists of an iPhone app and web-based therapy sessions, which work in conjunction, and which deliver treatment promptly in participants' daily lives. Chapter 4 contains a detailed description of the treatment protocol. The Think Slim webbased therapy sessions cover a series of CBT for obesity topics, such as coping with desire, formulating concrete diet goals, learning the CBT model for obesity, learning how to identify and evaluate dysfunctional cognitions, improving self-esteem and preparing for the posttreatment period. The Think Slim app contains three different modules of treatment. The first module provides passive feedback in the form of charts and graphs (Figure 3a). Second, the adaptive feedback module provides participants with warning messages and feedback whenever they are at risk for overeating (Figure 3b). The feedback reflects on what is taught during the online CBT sessions and asks participants to review their dieting goals and evaluated thoughts. Detection of risk is based on an automatic analysis of the EMA data of the participant involved (Spanakis, Weiss, Boh, \& Roefs, 2016). So, the app adjusts to participants' idiosyncratic experiences. Third, in case participants report a dysfunctional cognition, the thought evaluation module allows participants to evaluate this cognition and formulate a functional alternative cognition (Figure 3c). The acute effects of the Think Slim intervention are investigated in Chapter 5. The hypothesis is tested that Think Slim leads to more weight loss and more eating-related cognitive and emotional improvement than the self-guided diet-only control condition. In addition, follow-up measurements of the outcome measures will occur at 3 months and 12 months post-intervention. 
Part 1

Ecological momentary assessment of eating-related cognitions, emotions, food desire and food intake 



\section{Chapter 2}

Indulgent thinking? Ecological Momentary Assessment of Overweight and Healthy-weight Participants' Cognitions and

\section{Emotions}

B. Boh

A. Jansen

I. Clijsters

C. Nederkoorn

L. H. J. M. Lemmens

G. Spanakis

A. Roefs

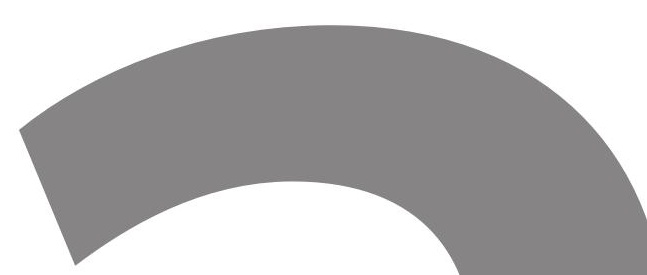

(2016). Indulgent thinking? Ecological Momentary Assessment of Overweight and Healthy-weight Participants' Cognitions and Emotions.

Behaviour Research and Therapy, 87, 196 - 206. 


\section{ABSTRACT}

Cognitions and emotions are considered important determinants of eating behaviour in cognitive behavioural models of obesity. Ecological data on these determinants is still limited. The present study investigated cognitions and emotions of overweight $(n=57)$ and healthy-weight ( $n=43$ ) participants via Ecological Momentary Assessment.

It was found that eating-related cognitions mainly focused on desire and taste. Unexpectedly, dysfunctional cognitions (i.e., thoughts that may promote overeating) did not occur more often for overweight participants in almost all cases. So, the present EMA study provides no evidence for a role of dysfunctional cognitions in obesity-promoting eating behaviour when assessing eating-related cognitions immediately prior to eating events using a free-text format assessment.

Right before eating events, participants mostly reported feeling calm/relaxed and cheerful/happy. Overweight participants scored higher on negative emotions, both at eating events and non-eating moments, than did healthy-weight participants. In addition, scores on standard questionnaires assessing emotional eating were positively associated with negative emotions reported at both eating and non-eating moments. As such, negative emotions, as assessed in the present study, do not seem to be specific triggers for food consumption. 


\section{INTRODUCTION}

The increased availability of high caloric palatable foods and drinks has been accompanied by a surge in obesity rates (Swinburn et al., 2011). Results of most weight loss trials have been marginal at best, with most or all weight-losses regained over a span of 5 years (Anderson, Konz, Frederich, \& Wood, 2001; Dansinger, Tatsioni, Wong, Chung, \& Balk, 2007; Douketis, Macie, Thabane, \& Williamson, 2005; Gudzune et al., 2015). One important reason for these disappointing results may be an insufficient focus on psychological factors, such as dysfunctional cognitions (i.e., thoughts that may promote overeating) and emotions, which may moderate the association between availability of food and eating behaviour (Carter \& Jansen, 2012; Faith, Fontaine, Baskin, \& Allison, 2007; Karhunen et al., 2012). The goal of the present study was to investigate cognitions and emotions as they occur when not eating, and prior to eating events in overweight and healthy-weight participants.

The cognitive model for obesity states that inadequate weight loss is the result of dysfunctional cognitions and emotions about eating, weight and shape (e.g., "when I eat, I have to empty my plate"; Werrij, 2005). Such cognitions can cause dietary disinhibition, and the resulting overeating in turn reinforces dysfunctional cognitions and beliefs (e.g., "I may as well continue eating"). Previous research has shown that obese participants had more dysfunctional food- and weight-related cognitions concerning catastrophizing, body image and self-control, than healthy-weight participants (Nauta et al., 2000; O'Connor \& Dowrick, 1987; Vreugdenburg et al., 2003). So far, the effectiveness of changing eatingrelated dysfunctional cognitions to promote weight loss has been investigated in several clinical trials. Studies comparing Cognitive (Behavioural) Therapy (C(B)T) for obesity with a diet and exercise control group (Werrij et al., 2009), behavioural therapy (Sbrocco et al., 1999), a wait-list control group (Stahre \& Hällström, 2007) and treatment-as-usual (Stahre, Tärnell, Håkanson, \& Hällström, 2007) found that the CBT group achieved modest weight loss that was maintained at a 12-month follow-up, whereas the other groups regained the lost weight. Cooper et al. (2010), however, found that weight lost after CBT was regained at 3-year follow-up. So, the evidence so far suggests CT/CBT might be relatively effective for the long-term treatment of obesity.

Another central cognitive-behavioural determinant of (over)eating for overweight people is emotions (Beck, 2011; Carter \& Jansen, 2012). Both positive and negative emotions have been associated with food intake (Cardi, Leppanen, \& Treasure, 2015; Oliver, Wardle, \& Gibson, 2000; van Strien, Herman, Anschutz, Engels, \& de Weerth, 2012). To improve CBT for obesity, more knowledge should be gained on the contents of dysfunctional cognitions and the nature of emotions prior to eating in overweight versus healthy-weight people (Carter \& Jansen, 2012).Therefore, the present study investigated cognitions and emotions as they occur when not eating, and prior to eating, using Ecological Momentary Assessment (EMA). 
EMA is an increasingly popular research method in which people's everyday lives are put under a magnifying lens (Mehl \& O'Connor, 2013). EMA forms an important adjunct to experimental research in laboratory settings, because it increases ecological validity (Shiffman, Stone, \& Hufford, 2008). EMA data can provide different insights from retrospective measures. For example, EMA may detect binge eating in cases where diagnostic interviews may not (Goldschmidt, Crosby, Cao, et al., 2014). EMA can also provide greater construct validity due to directly measuring relevant events (Shiffman et al., 2008). Given these advantages, EMA seems especially suitable for the investigation of cognitive and emotional associations with (problematic) eating behaviour.

So far, some studies have investigated eating- and obesity-related cognitions using EMA. It was found that all participants, but in particular those high on eating pathology, had increased exercise and diet-related cognitions after social comparisons (Leahey, Crowther, \& Ciesla, 2011; Rancourt, Leahey, Larose, \& Crowther, 2015). In addition, eating disordered participants had more negative emotion-related cognitions before and after binge eating events (Hilbert \& Tuschen-Caffier, 2007), and reported greater negative affect following self-reported loss of control after an eating event (Goldschmidt, Crosby, Cao, et al., 2014; Goldschmidt, Crosby, Engel, et al., 2014). Lastly, after a dietary lapse, women in a weight loss program reported more dieting and health-related cognitions than when merely tempted to eat (Carels, Douglass, Cacciapaglia, \& O'Brien, 2004). In short, so far, most EMA studies have covered a wide range of eating-related cognitions, mainly in samples with eating pathology.

For emotions, both negative and positive emotions coincided with eating events in healthy-weight participants (Macht, Haupt, \& Salewsky, 2004), and increases in these emotions were associated with more dietary lapses (Carels et al., 2001; McKee, Ntoumanis, \& Taylor, 2014). In adolescents, self-reported minor daily stressors predicted desire to eat highcaloric foods (Kubiak, Vögele, Siering, Schiel, \& Weber, 2008), and eating unhealthy snacks was associated with feeling more bored or lonely (Grenard et al., 2013). These data fit with laboratory studies showing that both negative and positive emotions can induce (over) eating in overweight (Chua, Touyz, \& Hill, 2004; Jansen et al., 2008) and healthy-weight participants (Bongers, Jansen, Havermans, Roefs, \& Nederkoorn, 2013; Oliver, Wardle, \& Gibson, 2000).

Previous EMA research on (over)eating behaviour mostly did not include comparisons between overweight and healthy-weight participants. In addition, most EMA research on eating-related cognitions has focused on eating disorders, and on BED in particular. However, the prevalence of BED is only around 30\% for individuals seeking weight loss treatment (de Zwaan, 2001). So, more insights into eating-related cognitions from a non-BED sample are needed. The present study examines cognitions and emotions in the context of eating events and non-eating moments in daily life, and compares overweight and healthy-weight participants. Cognitions and emotions were assessed over the course of a two-week period. Emotions and cognitions were rated immediately prior to self-reported eating events, and in 
the case of emotions, at random times throughout the waking day (non-eating moments). It was expected that overweight participants would report more dysfunctional cognitions and negative emotions prior to eating and would believe more strongly in such cognitions than healthy-weight participants.

A secondary aim was to investigate whether emotional eating questionnaire scores reflect actual emotional eating, or a dysfunctional belief about eating behaviour. Much research on emotional eating - defined as eating in response to experienced negative emotions - has relied on retrospective questionnaire measures, such as the Emotional Eating subscale of the Dutch Eating Behaviour Questionnaire (EE-DEBQ; van Strien et al., 1986). Several studies shed doubt on the validity of these self-report questionnaires. Self-reported emotional eaters did not eat more after negative emotions (Bongers, Jansen, Havermans, Roefs, \& Nederkoorn, 2013; O'Connor \& O'Connor, 2004). In addition, scores on the EE-DEBQ did not predict (snack) intake (Adriaanse et al., 2011; Bongers, de Graaff, \& Jansen, 2016, Bongers, Jansen, Houben, \& Roefs, 2013; Evers et al., 2009; Jansen et al., 2011; Lluch, Herbeth, Méjean, \& Siest, 2000; Werthmann et al., 2014). To further investigate the validity of emotional eating questionnaires, measurements of emotions and eating behaviour as they occur in daily life could be beneficial. This way, the issues of memory recall bias that are associated with retrospective questionnaire assessment (Schwarz, 2007; Bradburn, Rips, \& Shevell, 1987), especially for events with a high emotional impact (Fredrickson, 2000), are avoided. By comparing EMA and questionnaire data, it can then be established whether emotional eating is an actual eating style, or a dysfunctional belief about the association between emotions and eating (Adriaanse et al., 2011; Evers et al., 2009). Based on previous work (Adriaanse et al., 2011; Bongers et al., 2013; Evers et al., 2009), we did not expect a specific positive association between emotional eating questionnaire scores and high-caloric eating events, when experiencing negative emotions or reporting emotion-related cognitions. Instead, it was expected that such a positive association would occur at eating events and non-eating moments (i.e., at all assessments).

\section{METHOD}

\section{Participants and recruitment}

Participants were recruited via flyers that were spread throughout a university building, an academic hospital, health centres, local supermarkets, household fairs, and Facebook. Advertisements were also placed in local newspapers and on several websites. The study was approved by the Ethical Committee of the Faculty of Psychology and Neuroscience of Maastricht University. All participants signed an informed consent form.

Inclusion criteria for participation in the study were: (1) self-reported Body Mass Index (BMI) between 18.5 and 40, (2) in possession of an iPhone, (3) not on a professionally supervised 
diet, (4) no medical conditions that affect (regular) eating behaviour, and (5) not pregnant. Participants were matched on age, level of education and gender. Two participants provided self-report measures of BMI that were below 40, but turned out to have a BMI of 40.8 and 45.7 after obtaining supervised measurements. It was decided to leave these participants in the study, because of power considerations. The final sample consisted of 100 participants (see Table 1 for demographic data), with 57 overweight and 43 healthy-weight participants. Of the 57 overweight participants, 34 were considered 'overweight' (BMI between 25 and 30), 21 were considered 'obese' (BMI between 30 and 40), and 2 were considered 'morbidly obese' (BMI higher than 40). For the remainder of the article, all participants with BMI $>25$ will be referred to as 'overweight'. See Figure 1 for a flowchart of participation throughout the study.

Table 1 Demographics of the overweight $(n=57)$ and healthy-weight participants $(n=43)$ and relevant statistics.

\begin{tabular}{|c|c|c|c|c|}
\hline \multirow[b]{2}{*}{ Demographic } & \multirow{2}{*}{$\frac{\text { Overweight }}{\text { n }}$} & \multirow{2}{*}{$\frac{\text { Healthy-weight }}{\text { n }}$} & \multicolumn{2}{|c|}{ Comparison } \\
\hline & & & $x^{2}$ & $\mathbf{p}$ \\
\hline Gender & & & .000 & .99 \\
\hline Male & 7 & 5 & & \\
\hline Female & 50 & 38 & & \\
\hline Employment & & & 2.3 & .31 \\
\hline Unemployed & 10 & 4 & & \\
\hline Student & 18 & 19 & & \\
\hline Working & 29 & 20 & & \\
\hline Education & & & 1.7 & .65 \\
\hline No degree & 0 & 0 & & \\
\hline Lower & 10 & 5 & & \\
\hline Intermediate & 19 & 12 & & \\
\hline Higher & 17 & 14 & & \\
\hline \multirow[t]{2}{*}{ University } & 11 & 12 & & \\
\hline & $M(S D)$ & $M(S D)$ & $t(98)$ & $p$ \\
\hline Age & $31.2(10.0)$ & $32.1(10.6)$ & 0.43 & .67 \\
\hline BMI at measurement 1 & $30.3(4.3)$ & $22.1(1.5)$ & 13.4 & $<.0001^{*}$ \\
\hline BMI at measurement 2 & $30.2(4.2)$ & $22.2(1.5)$ & 13.2 & $<.0001^{*}$ \\
\hline
\end{tabular}

Note. $M=$ Mean, $S D=$ Standard Deviation, $n=$ Number of participants, $x^{2}=$ Chi Square. Neither the overweight participant group $(t(56)=1.22, p=.23)$ nor the healthy-weight participant group $(t(42)=1.07, p=.29)$ showed significant weight change between BMI measurement 1 and measurement 2 .

$*^{*}=p \leq 0.05$. 


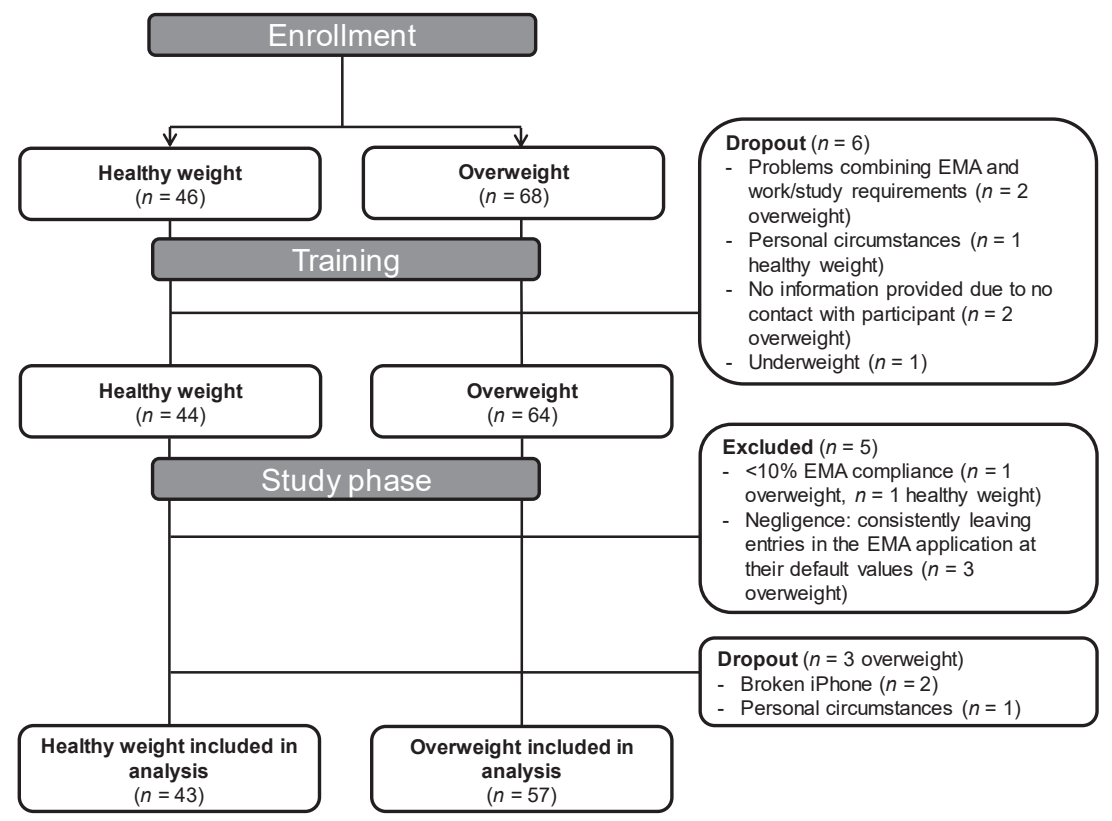

Figure 1 Flowchart of participants.

\section{Design of the EMA protocol}

The data presented in this paper are part of a larger EMA study on psychological factors related to eating behaviour. For the purpose of the overall study protocol of this larger study, an EMA app was developed for iPhone. The EMA protocol consisted of two parts: event sampling (assessment just before eating events) and signal-contingent sampling (assessments based around the time of day). Additionally, each day of sampling included a morning and evening questionnaire. The morning questionnaire assessed sleep quality and sleep duration. The evening questionnaire always occurred shortly before bedtime and assessed the intake of beverages. All self-report measures were provided in the Dutch language.

For the signal-contingent sampling, a waking day was, per default, divided into 8 time windows of two hours each. Time windows would be automatically added or removed depending on participants' sleep and waking times (as entered in the app). A signal-contingent sample occurred at a random time point within each time window (Hektner, Schmidt \& Czikszentmihalyi, 2006), and was triggered by a notification sent to the participant's iPhone. For eating event sampling, the participant was instructed to take his/her iPhone and press a button to initiate a brief EMA questionnaire whenever (s)he was about to eat something. At each assessment, the participant was asked to provide input on level of desire, specificity of desire, experienced emotions and physical location and circumstances. Completing an assessment took around 90 seconds. The eating event EMA questionnaire additionally assessed 
eating-related cognitions and the food that was about to be consumed. The EMA protocol lasted for two weeks in total.

To investigate the hypotheses of the present paper, only the emotion and cognition EMA questions of the signal-contingent and eating event-related samples were included in the analysis, along with the food consumption measure. Included emotion items were adapted from an earlier EMA study (Macht, Haupt, \& Salewsky, 2004), and included the following: 'angry/annoyed', 'anxious/scared', 'calm/relaxed,' 'cheerful/happy', 'sad/gloomy' and 'tense/stressed'. Additionally, the item 'bored' was included in this list because it was found to be an important determinant of eating in earlier research (Koball, Meers, Storfer-Isser, Domoff, \& Musher-Eizenman, 2012). Emotion items were presented to the participant as a list, led by the question "How do you feel?", and were individually scored on Visual Analogue Scales (VAS) ranging from 0 (not at all) to 100 (extremely). All emotion VAS items started by default at a score of zero. The participant was not required to fill in all VAS items one by one; instead (s)he had to choose at least one item that corresponded best to how (s)he felt at the time of assessment and then fill in the corresponding VAS. As such, the participant was allowed, but not obligated, to fill in multiple VAS items. The emotion VAS items that were not filled in by participants were considered to be equal to zero, also for the purposes of analysis. Note that this method, therefore, provides insight into the strongest experienced emotion(s) at the time of assessment.

Cognitions were assessed via a free-input text area in the app, in which the participant could write one current cognition, with the instruction to answer the question "What are you thinking regarding why you are going to eat right now?". The participant was instructed on how to answer this question prior to starting the EMA period in three ways. First, (s)he was provided with a printed EMA instruction that contained examples. Second, an instructional video series also contained an example of how to report cognitions. Finally, (s)he was asked over the phone if (s)he understood the instructions and any remaining questions were resolved. Examples of relevant cognitions include: "Everyone is eating cake, I might as well join in" / I am stuffed, but my mom cooked so I eat because it is respectful" / "Got some great news! I am celebrating!" / "I am just hungry and want to eat my lunch". The participant was explicitly instructed to give the cognition question careful consideration. Additionally, below the free text area, a separate question was asked: "How strongly do you believe in this thought?". The participant was instructed to specify his/her degree of belief on a VAS, ranging from 0 (not at all) to 10 (very much).

In addition to these questions, the participant ended the overall eating event EMA questionnaire by indicating what (s)he was about to eat. This was done by selecting out of a matrix of icons representing a wide selection of foods the icon(s) most closely resembling the food(s) that the participant was about to eat. Also, the participant took a picture of his/ her food, which was used to verify that an actual eating event was about to take place. Once an EMA questionnaire had been completed, it appeared in an overview list and could be reviewed as a brief summary. To minimize reactivity to the protocol, this review option contained only minimal information. 


\section{Questionnaires}

\section{Screening questionnaire}

Interested candidates were sent a screening questionnaire that included questions about age, sex, dieting status, current employment, education level, weight and height, pregnancy, menopause, current medical and psychological treatment, dietary requirements, experience with monitoring eating behaviour, and the possession of an iPhone.

\section{Post-EMA period questionnaire battery}

After the EMA period ended, the participant filled in a battery of questionnaires included the emotional eating subscale of the Dutch Eating Behaviour Questionnaire (EE-DEBQ; van Strien et al., 1986), the Emotional Eating Questionnaire (EES; Arnow et al., 1995), the Problematic Eating Behaviour Questionnaire (Carter \& Jansen, 2012), the Belief in Dysfunctional Thoughts Scale (Werrij et al., 2009), and a brief feedback questionnaire on the use of the app. The participant completed these questionnaires in the week following the EMA period. Questionnaires were administered after the EMA-period to avoid the influence of questionnaire-assessment on the EMA data. For the analyses reported in the present paper, only data from the EE-DEBQ and the EES are discussed.

The DEBQ (van Strien et al., 1986) is a 33-item questionnaire that assesses different types of eating styles (subscales of the questionnaire): emotional eating, restrained eating, and external eating. Items are rated on a 5-point Likert scale ranging from 'never' to 'very often'. The EE-DEBQ subscale consists of 13 items. The item-total correlations of the subscale were previously found to be $>0.65$ (van Strien et al., 1986). The subscale also had a high internal consistency in the current sample $(a=.94)$.

The EES (Arnow et al., 1995) is a questionnaire that consists of 25 items that describe emotional states, with 5-point Likert-scale options going from "no desire to eat" to "an overwhelming urge to eat". People are requested to indicate the extent to which the 25 items apply to their own food desire experiences. The test-retest reliability for this questionnaire was previously found to be sufficient; $r=.79, p<0.001$ (Arnow et al., 1995). The questionnaire was translated to Dutch by the authors of the present study and had a high internal consistency in the current sample $(a=.95)$.

\section{Weight and height measurement}

A first measurement of height and weight was conducted in the week prior to the start of the training. The second measurement was conducted during the week after the EMA period, and included only weight). Participants were instructed to undergo supervised measurements, either externally (conducted by health care professionals such as general practitioners and physiotherapists) or at the university (conducted by the researcher or research assistants), both times under identical conditions (same scale and supervisor). Eighteen 
participants underwent both measurements at the university, whereas 71 participants went to a healthcare professional. The remaining 11 participants ( $n=7$ overweight, $n=4$ healthyweight) reported being unable to obtain one or both supervised measurements (e.g., due to busy work schedules, or private circumstances). These participants were allowed to provide self-report measurements to avoid study drop-out.

\section{Procedure}

All e-mail and phone correspondence was standardized. Interested candidates were sent a link to an online screening questionnaire $\left(X_{0}\right)$ via e-mail $\left(@_{0}\right)$. Those eligible for inclusion were contacted via e-mail $\left(@_{1}\right)$ to plan a suitable two-week period of participation. After inclusion, the participant was contacted by phone $\left(\mathbf{s}_{1}\right)$ and instructed to complete a brief questionnaire $\left(X_{1}\right)$ and obtain a first measurement of BMI (BMI1). Additionally, an instruction guide for the study and app was sent via postal mail ( $\times)$. The participant was also provided with a username and identification code to ensure confidentiality. Participants were clearly instructed that their assessments were kept confidential.

Next, the participant was contacted via e-mail $\left(@_{3}\right)$ and telephone $\left(\mathbf{s}_{2}\right)$ to schedule a oneday training with the app and EMA protocol. After completing the training, the participant was contacted via phone to discuss his/her performance $\left(\mathbf{s}_{3}\right)$. The training was repeated $(n=4)$ if the participant (1) did not report any eating events, (2) did not report any signalcontingent samples, or (3) misunderstood the instructions and thought that prompts to complete signal-contingent samples were prompts to eat something. The two-week EMA period started at the Monday of the subsequent week to ensure that the number of weekend and weekdays was equal across participants. The participant was then sent an e-mail $\left(@_{4}\right)$ with this information.

During the two-week EMA period, the participant used the app and was allowed to contact experimenters in case of technical difficulties or interfering personal circumstances. At day 3 or 4 , the participant was briefly contacted via phone $\left(\mathbf{s}_{4}\right)$ for a check-up. At day 8 , a brief motivational e-mail $\left(@_{5}\right)$ was sent. At the end of the EMA period, the participant was asked via phone $\left(\mathbf{s}_{5}\right)$ to undergo a second measurement (BMI2) and fill out the post-EMA questionnaire $\left(\mathrm{X}_{2}\right)$. The participant was also debriefed about the study goals. Upon handing over the results of the second measurement of weight and completing the post-EMA questionnaire, the participant received a gift certificate of $€ 50$,-, and was sent a final e-mail (@ $\left.{ }_{7}\right)$.

\section{Data analysis}

The main focus of the present paper was on a comparison between overweight and healthyweight participant groups, so change over time within individuals was not considered in the analyses. Instead, the EMA methodology provided the benefit of repeatedly assessing cognitions and emotions immediately prior to eating events and non-eating moments in daily life. As suggested by Shiffman (2014), data were collapsed across all assessment 
points, and the aggregated or averaged values were entered in between-subjects analyses. This increased the reliability of the cognition and emotion measurements (Shiffman, 2014). Note that time-lagged analyses were reported in a different paper on this dataset (Spanakis, Weiss, Boh, \& Roefs, 2016), which focused on predicting eating behaviour at time-point $t$ from data gathered at time-point $t-1$.

As a first step in the analyses, assessments were divided into three types. One assessment type consisted of eating events that included the selection of at least one high-caloric food, such as snack foods and dishes with a side of French fries. The second type consisted of the remainder of foods, which were lower in caloric content and relatively healthier options, such as fruits and vegetables and dishes with a side of rice or potatoes (healthier option foods). The last type consisted of all the signal-contingent samples (non-eating moments). Table 2 contains an overview of food products included in the high-caloric and healthier option assessment types.

As a second step in the analyses, cognitions were assigned to categories according to theme. To this end, a subset of 700 cognitions (350 of overweight participants and 350 of healthy-weight participants) was randomly selected from the total dataset. Then, three of

Table 2 Overview of food products assigned to the high-caloric and to the healthier option eating event assessment types. Note that participants were instructed to select one or multiple food products most closely resembling what they were about to eat.

\begin{tabular}{|c|c|}
\hline Food products & Included in eating event type \\
\hline $\begin{array}{l}\text { Hamburger, Muffin, Cookies, Candy Bar, Chips, Cake, } \\
\text { Ice-cream, Pizza, Dish with a side of fries }\end{array}$ & High-caloric \\
\hline $\begin{array}{c}\text { Sandwich, Yoghurt, Cornflakes, Salad, Fruit, Soup, Pasta } \\
\text { dish, Rice dish, Dish with a side of potatoes }\end{array}$ & Healthier option \\
\hline
\end{tabular}

the researchers (AJ, AR and BB) determined appropriate categories that could apply to all 700 cognitions in the subset. This resulted in three main categories: 'functional','neutral' and 'dysfunctional'. These categories were each further split into several subcategories. The exact categories and subcategories can be found in Table 3. Next, two blinded independent raters (a CBT therapist (LL) and a researcher who was not involved in the present study) and one of the researchers (BB) used the proposed list of categories to categorize another randomly generated subset of 700 cognitions. In clearly ambiguous cases of multiple subcategories being applicable (for example: "I am hungry, because I feel bad"), raters were instructed to prioritize the category of dysfunctional cognitions over the category of neutral cognitions.

Next, an interrater reliability analysis was performed using Fleiss's Kappa to determine the consistency among the three raters, with Kappa $=0.76(p<0.001), 95 \% \mathrm{Cl}[0.73,0.79]$ for healthier option foods, and Kappa $=0.71(p<0.001), 95 \% \mathrm{Cl}[0.68,0.74]$ for high-caloric foods. A Fleiss's Kappa value between $0.61-0.80$ reflects 'substantial agreement' (Landis \& 
Koch, 1977). Finally, one of the researchers (BB) blindly categorized all 5521 cognitions using these categories. This final categorization was then applied to subsequent data analyses.

\section{RESULTS}

\section{Compliance ratings for the EMA entries}

The percentage of completed non-eating moment (signal-contingent) assessments relative to the total number of signal-contingent assessments participants received during the 14day EMA period was calculated. Overweight participants completed 80.91\% (SD = 9.96\%) of the assessments, and healthy-weight participants completed $79.73 \%$ (SD $=9.73 \%$ ) of the assessments. Compliance did not differ between both participant groups, $t(98)=0.59$, $p=0.55$. Furthermore, overweight participants reported $M=3.77(S D=1.05)$ eating events on average per day, whereas healthy-weight participants reported $M=4.17$ (SD $=1.50$ ) eating events on average per day.

\section{Cognitions preceding eating events}

During the two weeks of EMA, 5521 unique cognitions were obtained. There was no difference in the number of reported cognitions, averaged over participants, between overweight and healthy-weight participants, $t(98)=1.59, p=0.12$ (overweight: $M=52.77, S D=14.71$; healthy-weight: $M=58.44, S D=21.03$ ). Note that this also means there were no group differences in the average number of reported eating events, because only one cognition entry was provided by participants per assessment.

In total, 4007 cognitions were allocated to the healthier option foods assessment type, and 1514 cognitions were allocated to the high-caloric foods assessment type. Healthyweight participants on average reported significantly more healthier option eating events than did overweight participants, $t(98)=2.26, p=0.03$ (healthy-weight: $M=43.4, S D=14.9$; overweight: $M=37.6, S D=10.8$, whereas no group difference was found for the high-caloric foods, $t(98)=0.06, p=0.95$ (healthy-weight: $M=15.1, S D=10.8$; overweight: $M=15.2$, $S D=10.0)$.

\section{Differences for cognitions between overweight and healthy-weight participants}

Frequency of occurrence of cognitions was compared between overweight and healthyweight participants for all the cognition subcategories, separately for the high-caloric and healthier option foods. Considering that the number of high-caloric and healthier option eating event types differed over participants, the frequency of occurrence of each subcategory of cognitions was expressed as a percentage of the total number of cognitions per 


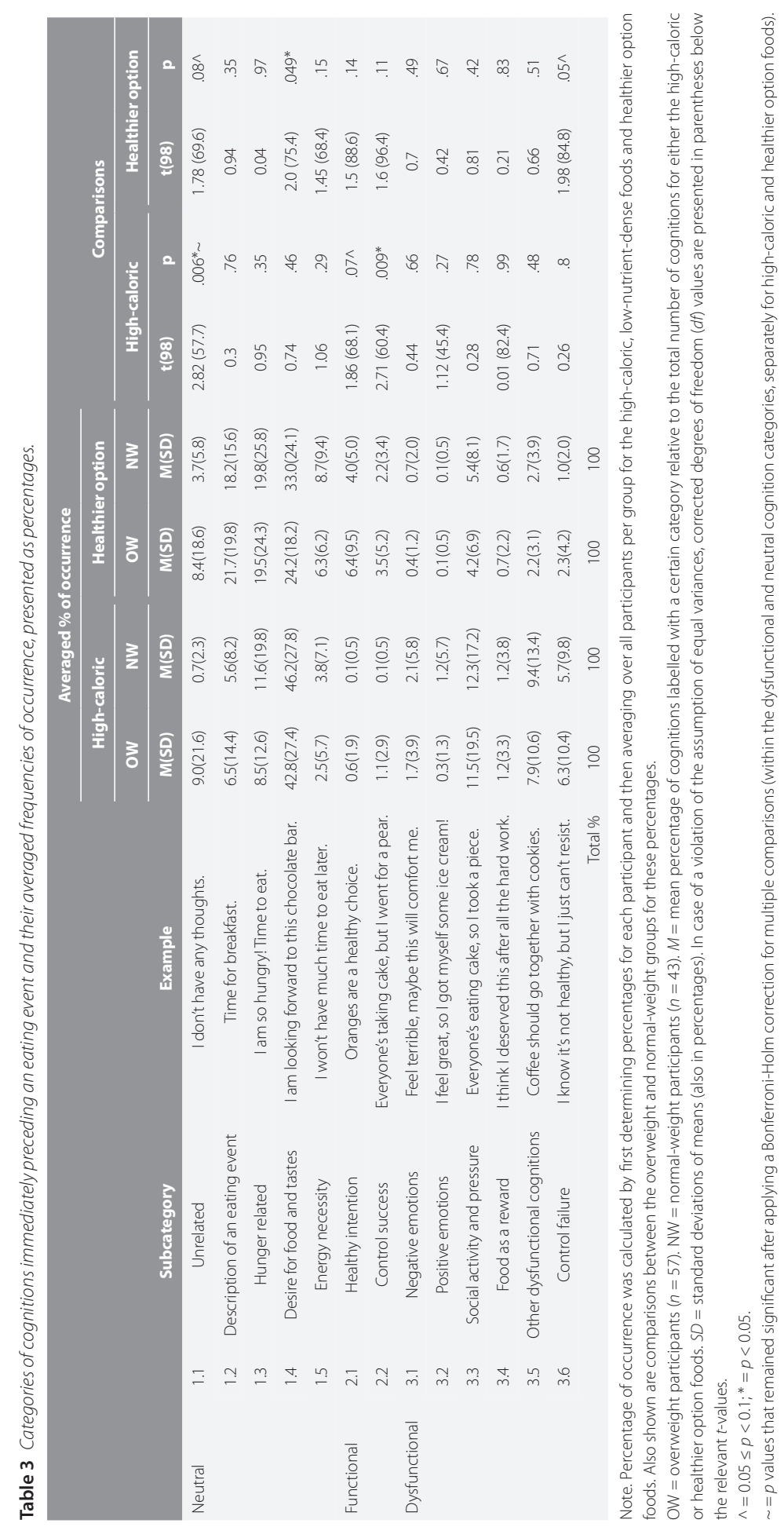




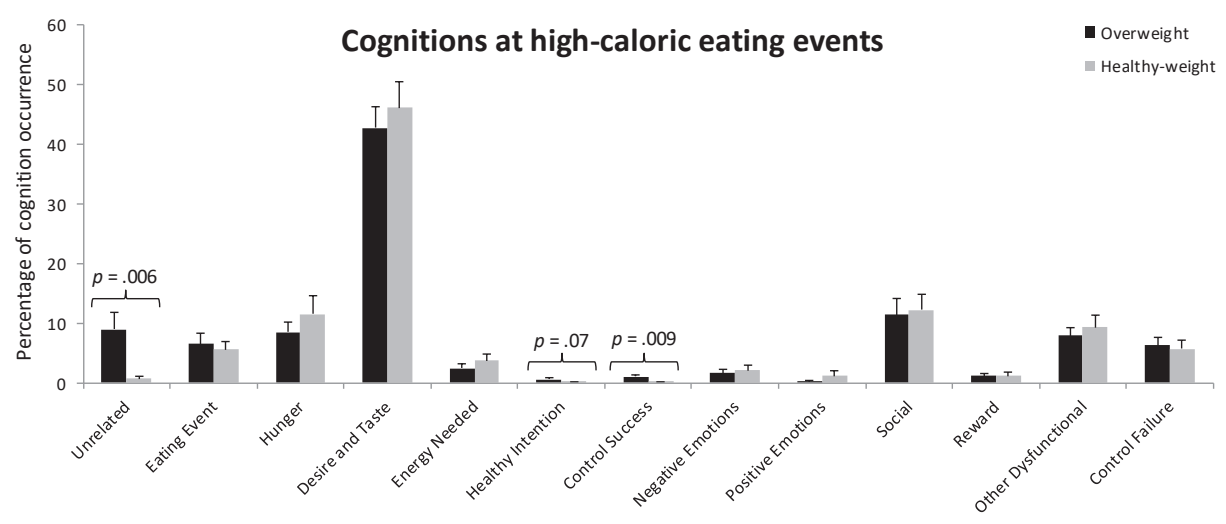

Figure 2a Bar plots of overweight and healthy-weight participants' cognitions related to the high-caloric foods. Note. The $y$-axis represents percentages of frequency of occurrence of cognition subcategories for high-caloric foods, relative to the total number of cognitions for these foods. Error bars represent standard errors.

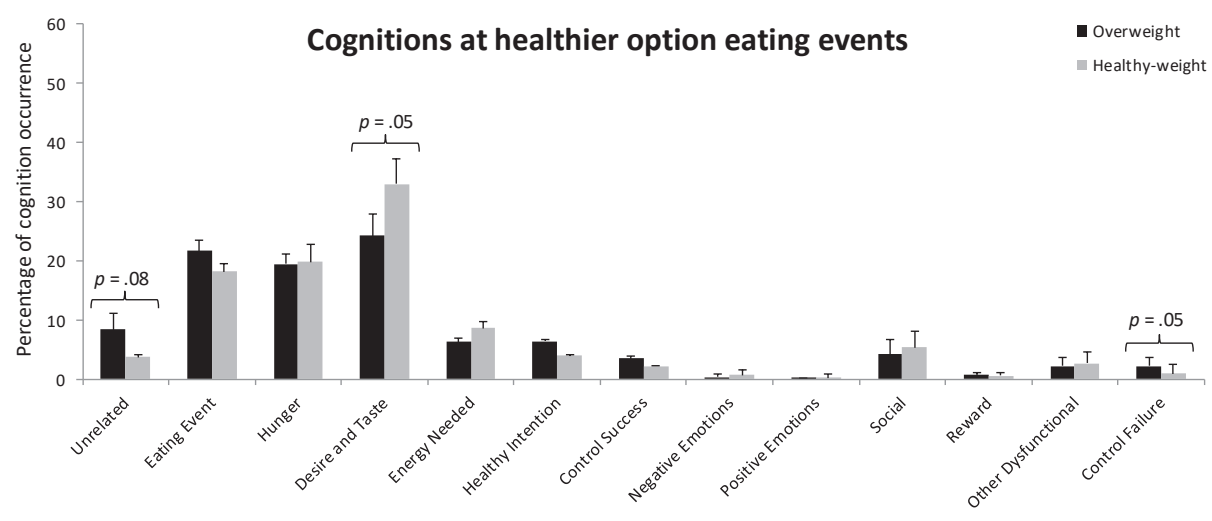

Figure $\mathbf{2 b}$ Bar plots of overweight and healthy-weight participants' cognitions related to the healthier option foods. Note. The $y$-axis represents percentages of frequency of occurrence of cognition subcategories for healthier option foods, relative to the total number of cognitions for these foods. Error bars represent standard errors.

eating event assessment type (high-caloric and healthier option foods) per participant. These percentages were then averaged over participants, separately for the overweight and healthy-weight groups. Table 3 and Figure 2 contain an overview of the obtained results.

For the neutral cognitions, overweight participants reported more eating-unrelated cognitions for high-caloric foods than healthy-weight participants, with a trend-significant group-difference for healthier option foods. Healthy-weight participants reported more desire and taste cognitions for healthier option foods than overweight participants. For the

1 Analyses of differences between overweight and healthy-weight participants using raw frequency totals per food type per participant group did not lead to a different pattern of results. 
functional cognitions, overweight participants reported more successful control cognitions and trend-significantly more healthy intention cognitions for the high-caloric foods when compared to healthy-weight participants. However, the number of these cognitions was relatively low. For the dysfunctional cognition category, only control failure cognitions were reported significantly more by overweight participants relative to healthy-weight participants for healthier option foods. Importantly, obtained $p$-values for the neutral and dysfunctional cognition categories were adjusted for multiple comparisons via a stepwise rejective Bonferroni-Holm correction (Holm, 1979), separately for high-caloric and healthier option foods. Only the difference between overweight and healthy-weight participants of the occurrence of eating-unrelated cognitions for high-caloric foods remained significant after these corrections.

In addition to frequency of occurrence, belief in reported dysfunctional cognitions was investigated. Because many of the dysfunctional cognition subcategories did not occur for the majority of participants, it was decided to investigate belief in all dysfunctional cognition subcategories taken together (i.e., belief scores for dysfunctional cognitions were summed and then averaged irrespective of subcategory), separately for the high-caloric and healthier option food types. Results showed that overweight participants did not differ from healthyweight participants in their belief of dysfunctional cognitions for the high-caloric foods (healthy-weight $M=6.74, S D=1.49$; overweight $M=6.77, S D=1.58), t(49)=0.06, p=0.95$, nor for the healthier option foods (healthy-weight $M=6.71, S D=1.54$; overweight $M=6.72$, $S D=1.39), t(49)=0.03, p=0.98$.

\section{Emotions preceding eating events and non-eating moments}

By far the highest overall scores were found for the 'cheerful / happy' and 'calm / relaxed' emotions, irrespective of assessment type. 2 (group: overweight vs. healthy-weight) By 3 (assessment type: high-caloric foods, healthier option foods, non-eating moments) mixed ANOVAs were performed for each of the seven emotions separately. Greenhouse-Geisser corrected $F$-values are reported, and statistics relating to main effects can be found in Table 4. If there was a main effect of assessment type, three pairwise comparisons were performed pooling over participant group. Only for 'sad / gloomy', a trend-significant participant group $x$ assessment type interaction emerged. Contrary to expectations, Separate 1-way ANOVAs for overweight and healthy-weight participants revealed a significant effect of assessment type for healthy-weight participants, $F=5.88, p=0.01$, but not for overweight participants, $F=0.43, p=0.57$. Pairwise comparisons of assessment type for the healthy-weight group indicated that healthy-weight participants were trend-significantly more 'sad / gloomy' at healthier option eating events ( $p=0.06$ ) and significantly more'sad / gloomy' at non-eating moments ( $p=0.02$ ) compared to high-caloric eating events.

Looking at the pattern of results in Table 4 (main effects), it appeared that overweight participants were always more 'sad / gloomy' and (as a trend) more 'tense / stressed' than 


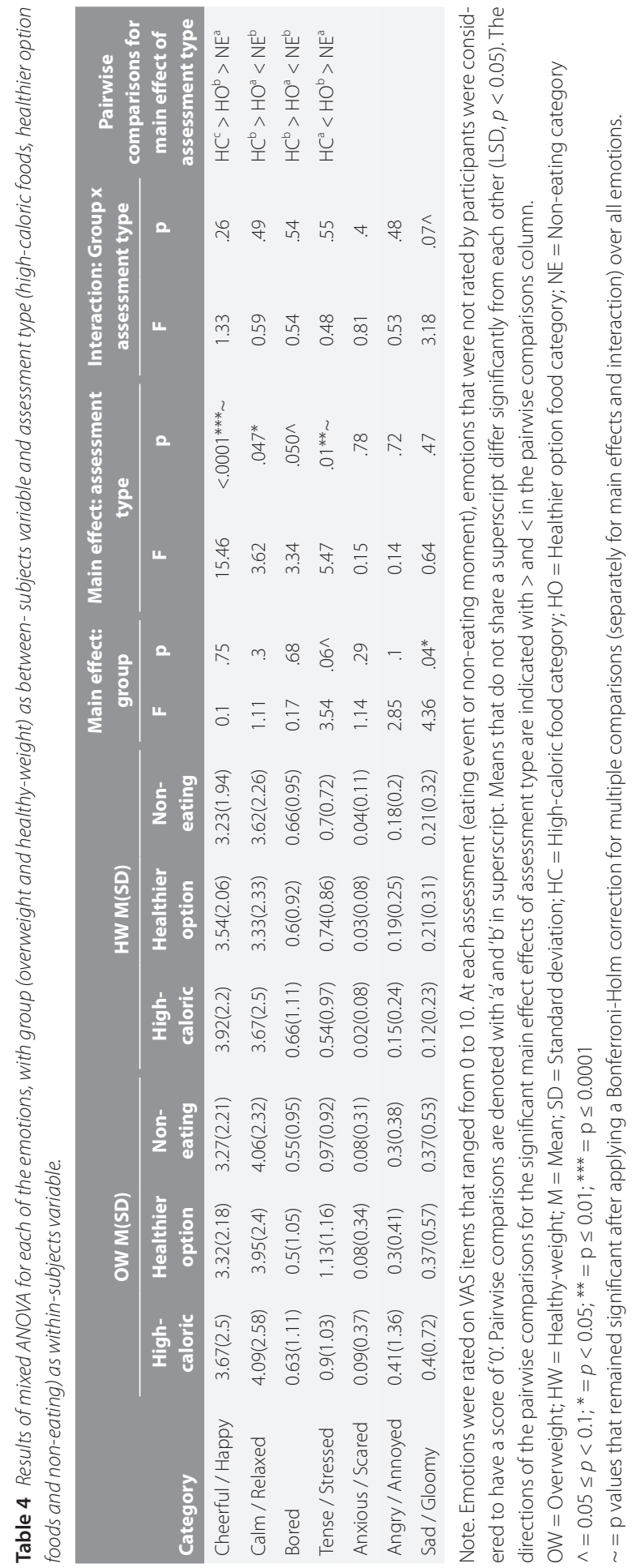


healthy-weight participants, independent of assessment type. Furthermore, pairwise comparisons between each of the assessment types revealed that overall participants were most cheerful when about to eat, especially if the food was high-caloric. Participants were least 'calm / relaxed' and 'bored' and, contrary to expectations, most 'tense / stressed' at healthier option eating events compared to high-caloric eating events and non-eating moments. Scores for high-caloric eating events and non-eating moments did not differ for these three emotions.

\section{EE-DEBQ and EES scores in comparison with emotion-related cognitions and emotions}

Overweight participants scored trend-significantly higher than did healthy-weight participants on the $E E-D E B Q, t(98)=1.82, p=0.07$ (healthy-weight: $M=2.17, S D=0.79$; overweight: $M=2.5, S D=0.99)$, but there was no group-difference for the EES, $t(98)=1.31, p=0.19$ (healthy-weight: $M=47.56, S D=16.14$; overweight: $M=52.68, S D=21.49$ ). The EE-DEBQ and EES correlated significantly, $r(98)=0.84, p<0.001$.

Next, EE-DEBQ and EES scores were correlated with the frequencies of occurrence of the negative and positive emotion cognition subcategory and emotion scores, for the highcaloric and healthier option food categories separately, and for non-eating moments. See Table 5 for an overview of the correlation results. For emotion cognitions, both negative and positive emotion cognitions, irrespective of food type (high-caloric or healthier option), correlated positively with the EE-DEBQ and EES. Furthermore, negative emotion scores (except

Table 5 Correlations of the emotional eating subscale of the Dutch Eating Behaviour Questionnaire (EE-DEBQ) and the Emotional Eating Scale (EES) with emotion-related cognitions and emotion scores, both for eating events (high-caloric and healthier option) and non-eating moments.

\begin{tabular}{|c|c|c|c|c|c|c|c|c|c|c|c|c|}
\hline \multirow[b]{3}{*}{ Category } & \multicolumn{6}{|c|}{ Correlations with the EE-DEBQ (n=99) } & \multicolumn{6}{|c|}{ Correlations with the EES (n=99) } \\
\hline & \multicolumn{2}{|c|}{ High-caloric } & \multicolumn{2}{|c|}{$\begin{array}{c}\text { Healthier } \\
\text { option }\end{array}$} & \multicolumn{2}{|c|}{ Non-Eating } & \multicolumn{2}{|c|}{ High-caloric } & \multicolumn{2}{|c|}{$\begin{array}{c}\text { Healthier } \\
\text { option }\end{array}$} & \multicolumn{2}{|c|}{ Non-Eating } \\
\hline & $\mathbf{r}$ & $\mathbf{p}$ & $\mathbf{r}$ & $\mathbf{p}$ & $\mathbf{r}$ & $\mathbf{p}$ & $\mathbf{r}$ & $\mathbf{p}$ & $\mathbf{r}$ & $\mathbf{p}$ & $\mathbf{r}$ & $\mathbf{p}$ \\
\hline \multicolumn{13}{|l|}{$\underline{\text { Cognitions }}$} \\
\hline Negative emotions & 0.27 & $.007^{*}$ & 0.17 & .13 & & & 0.34 & $.001^{*}$ & 0.22 & $.03^{*}$ & & \\
\hline Positive emotions & 0.19 & $.06 \wedge$ & 0.19 & $.07 \wedge$ & & & 0.21 & $.04^{*}$ & 0.1 & .32 & & \\
\hline \multicolumn{13}{|l|}{ Emotions } \\
\hline Anxious / Scared & 0.14 & .18 & 0.14 & .15 & 0.15 & .14 & 0.16 & .11 & 0.16 & .12 & 0.16 & .11 \\
\hline Angry / Annoyed & 0.19 & $.07 \wedge$ & 0.2 & $.050^{\wedge}$ & 0.14 & .17 & 0.24 & $.016^{*} \sim$ & 0.15 & .15 & 0.08 & .42 \\
\hline Sad / Gloomy & 0.24 & $.02^{*}$ & 0.22 & $.03^{*}$ & 0.26 & $.01^{*}$ & 0.25 & $.01^{*} \sim$ & 0.2 & $.050^{\wedge}$ & 0.26 & $.01 *$ \\
\hline Tense / Stressed & 0.22 & $.03^{*}$ & 0.15 & .14 & 0.16 & .13 & 0.3 & $.002^{*} \sim$ & 0.25 & $.045^{*}$ & 0.22 & $.03^{*}$ \\
\hline Cheerful / Happy & -0.14 & .17 & -0.1 & .3 & -0.06 & .58 & -0.05 & .64 & 0.01 & .95 & 0.04 & .7 \\
\hline Calm / Relaxed & -0.16 & .12 & -0.18 & $.07 \wedge$ & -0.17 & $.09 \wedge$ & -0.16 & .1 & -0.16 & .11 & -0.17 & .12 \\
\hline Bored & 0.03 & .78 & -0.01 & .95 & -0.02 & .84 & 0.07 & .49 & 0.05 & .63 & 0.01 & .94 \\
\hline
\end{tabular}

Note. Emotion scores ranged between 0 and 10. The presented emotion categories are translated from Dutch.

$\wedge=0.05 \leq p<0.1 ;{ }^{*}=p<0.05$.

$\sim=$ p values that remained significant after applying a Bonferroni-Holm correction for multiple comparisons (separately for high-caloric eating events, healthier option eating events and non-eating moments) over all emotions. 
'scared / anxious' and 'bored'), irrespective of type of eating event, also correlated positively with the EE-DEBQ and EES. For positive emotion scores, there was a trend-significant negative correlation of 'calm / relaxed' with the EE-DEBQ for healthier option foods. Interestingly, for non-eating moments, negative emotions ('sad / gloomy' and 'tense / stressed') also correlated positively with the EE-DEBQ and EES, whereas the positive emotion 'calm / relaxed' correlated trend-significantly negatively with the EE-DEBQ only. So, overall, participants scoring high on the EE-DEBQ or the EES also scored higher on scores for several mainly negative emotions at both eating events and non-eating moments and on the occurrence of emotion-related cognitions at eating events than participants scoring low on these questionnaires.

\section{DISCUSSION}

The present study investigated how cognitions and emotions experienced in daily life relate to eating behavior in overweight and healthy-weight participants. Contrary to expectations, overweight and healthy-weight participants were largely comparable in terms of frequencies of, and belief in, cognitions at eating events. The most important differences were that overweight participants reported more eating-unrelated cognitions at high-caloric eating events, whereas healthy-weight participants reported more desire and taste cognitions at healthier option eating events. As for emotions, during eating events and non-eating moments, both participant groups mainly reported feeling neutral to positive, with overweight participants and self-reported emotional eaters being more negative irrespective of whether they were eating or not. That is, contrary to our hypotheses, emotions did not specifically predict eating events for overweight participants and self-reported emotional eaters.

Participants indicated mostly that they ate because of a desire to experience taste and give in to desire for food. The number of dysfunctional cognitions was relatively low in comparison with the number of neutral/functional cognitions and barely differed between overweight and healthy-weight participants. Belief in dysfunctional cognitions also did not differ for overweight and healthy-weight participants. So, dysfunctional cognitions did not appear to play a large role in overweight participants' eating behavior. This is not in line with cognitive (behavioral) models for obesity (Beck, 2007; Cooper \& Fairburn, 2001; Werrij, 2005), which state that in particular dysfunctional cognitions are important determinants of overeating behavior.

Importantly, the number of reported dysfunctional cognitions may have been underrepresented in the present study. Firstly, the present study only assessed eating-related cognitions immediately prior to eating events, with the foods being in front of the participant. However, deciding to eat is the result of many separate decisions (Wansink \& Cashman, 2007) that do not just take place immediately prior to eating. It may be that dysfunctional 
cognitions play a more important role at earlier stages in the decision-making process. Secondly, being about to eat, and being able to see and smell the food, may have promoted desire and taste-related cognitions to such a degree that these would take precedence over dysfunctional cognitions. It might therefore be interesting to investigate dysfunctional cognitions that occur during moments of dietary weakness (Carels et al., 2001; Mckee et al., 2014) and by giving participants the option to report multiple cognitions at an assessment. Thirdly, the low occurrence of dysfunctional cognitions could be due to the set-up of the study. In therapy settings, clients are first trained to identify (automatic) cognitions. This may be an essential prerequisite for effective reporting of cognitions. So, overall, the assessment of eating-related cognitions could be expanded by measuring at different time points and by training participants in recognizing their cognitions beforehand.

For the differences between overweight and healthy-weight participants related to specific cognitions, only the difference for the eating-unrelated cognitions subcategory remained significant after a multiple comparisons correction. This cannot plausibly be explained by a difference in cognitive abilities between participant groups, as participants were matched on level of education. Instead, it may be that overweight participants (1) were less willing to be confronted with their actual cognitions about eating, (2) were less likely to commit the effort to report cognitions, or (3) actually had fewer eating-related cognitions than healthy-weight participants. This third interpretation is in line with the idea that eating is often an automated, habitual, behavior (Cohen \& Farley, 2008). Another finding that is important because of the prevalence of desire and taste-related cognitions, is that healthy-weight participants reported more of such cognitions at healthier option eating events. Overweight participants are more sensitive to the rewarding properties of foods (Davis, Strachan, \& Berkson, 2004). Thus, it might be that healthy-weight participants appreciated the taste of these healthier option foods more than overweight participants.

The present study also investigated the association between emotions and eating behavior. For the majority of assessments, healthy-weight and overweight participants reported feeling neutral to positive. Both participant groups were most positive when about to eat high-caloric foods, followed by healthier option foods and then non-eating moments. Relatedly, for both participant groups, low-arousing emotions ('calm / relaxed', 'bored') were most strongly associated with high-caloric foods and non-eating moments, whereas highlyarousing emotions ('tense / stressed') were most strongly associated with healthier option foods. These findings provide further evidence for the concept of 'happy eating' (Bongers, Jansen, Havermans, et al., 2013; Bongers, Jansen, Houben, et al., 2013; Evers, Adriaanse, de Ridder \& de Witt Huberts, 2013), which states that experiencing more positive emotions is associated with increased caloric intake. In addition, it seems that low-arousing emotions are also important antecedents of snacking.

Most strikingly, overweight participants scored higher on negative emotions than did healthy-weight participants on all three assessment types (high-caloric foods, healthier 
option foods and non-eating moments). This suggests that overweight participants were generally more emotionally negative than healthy-weight participants. So, negative emotions do not appear to be specifically associated with the intake of high caloric foods in overweight people. Instead, overweight people reported more negative emotions in general than did healthy-weight people. This is contrary to what some studies have reported (Oliver, Wardle, \& Gibson, 2000; van Strien, Herman, Anschutz, Engels, \& de Weerth, 2012). However, those studies relied on retrospective questionnaires to assess the association between emotions and eating. In line with the hypothesis of the present study's secondary aim, emotional eating questionnaire scores were associated with negative emotions at all assessments, irrespective of eating. This provides further evidence for the argument that people may hold a dysfunctional belief about the presence of such an association, which is not supported by actual eating behavior behavior (Adriaanse et al., 2011; Bongers et al., 2013; Evers et al., 2009).

The present results are complementary to an analysis of the time-lagged (with lag $t-1$ ) network structure of these data (Spanakis et al., 2016). In these time-lagged analyses, it was found that the interplay between emotions and eating events was more complex and dense in overweight participants, than in healthy-weight participants. In addition, negative emotions, high-caloric food desires and eating events played a more central role in the time-lagged networks for overweight participants than for healthy-weight participants. Furthermore, for all participants, positive emotions at time $t-1$ promoted eating something high-caloric at time $t$. Interestingly, only for overweight participants, negative emotions at time $t-1$ specifically promoted high-caloric eating events, and inhibited healthier option eating events, at time $t$. This pattern was not observed for healthy-weight participants. These findings differ from the cross-sectional analyses presented in the present paper, in which overweight participants seemed to be more emotionally negative in general as compared to healthy-weight participants. So, a negative emotion experienced some time before an eating event seems to be more relevant for predicting specific eating behavior than a concurrently experienced emotion.

A potential limitation of the present study is the selective influence of repeated assessments of eating events on (determinants of) eating behavior of overweight participants. It is possible that being repeatedly asked to report eating events may have made overweight participants more weight-conscious. Note that most studies investigating this issue did not find any influence of reactivity on data quality (Cruise, Broderick, Porter, Kaell, \& Stone, 1996; Heron \& Smyth, 2013; Stone et al., 2003), although one study did (Fuller-Tyszkiewicz et al., 2013). In the present study, to minimize assessment reactivity, the duration of completing an assessment was deliberately kept short, at around 90 seconds.

Another potential limitation of the current study is that socially desirable answering tendencies may have influenced the results. Being overweight is met with social stigma (Puhl \& Brownell, 2003). So, overweight participants may not have been willing to report 
all high-caloric eating events, or dysfunctional cognitions. For eating events, this issue can only be overcome by using covert monitoring, instead of relying on self-report measures. However, there is no easy, and ethical, solution to achieve this. For the assessment of internal constructs, such as cognitions and emotions, one has no other option than to rely on introspection (McNally, 2001).

In conclusion, in the present study, no support was found for the importance of dysfunctional cognitions as determinants of overeating behavior (Carter \& Jansen, 2012). Instead, cognitions were mainly related to desire and taste. Moreover, the experience of negative emotions does not seem to be specifically related to the consumption of high-caloric foods in overweight participants. Instead, overweight participants reported more negative emotions, independent of whether it was an eating event (high-caloric or healthier option) or not, than healthy-weight participants. Similarly, participants scoring high on questionnaires (EE-DEBQ / EES), reported more negative emotions regardless of whether they were eating or not. Note that these conclusions are limited by the assessment of emotions and cognitions immediately preceding an eating event. Future research should focus on exploring cognitions at earlier time points in the decision making process about whether or not to eat (i.e., during moments of dietary weakness).

\section{ACKNOWLEDGMENTS}

This research was supported by grant 12028 from Stichting Technische Wetenschappen (STW), Nationaal Initiatief Hersenen en Cognitie (NIHC), Nederlandse Organisatie voor Wetenschappelijk Onderzoek (NWO) and Philips under the Partnership programme Healthy Lifestyle Solutions to the last author. The authors are grateful to Vincent Kerkhofs for his work on programming the app used in the present study, and to Jettie Hoonhout and Marieke Wichers for valuable discussions on the study's design. 



\title{
Chapter 3
}

\section{Food Desire in Daily Life:}

Comparison of overweight and

healthy-weight participants via ecological momentary assessment

\author{
B. Boh \\ A. Jansen \\ C. Nederkoorn \\ G. Spanakis \\ A. Roefs
}

(in revision). Food desire in daily life:

comparison of overweight and healthy-weight participants via ecological momentary assessment.

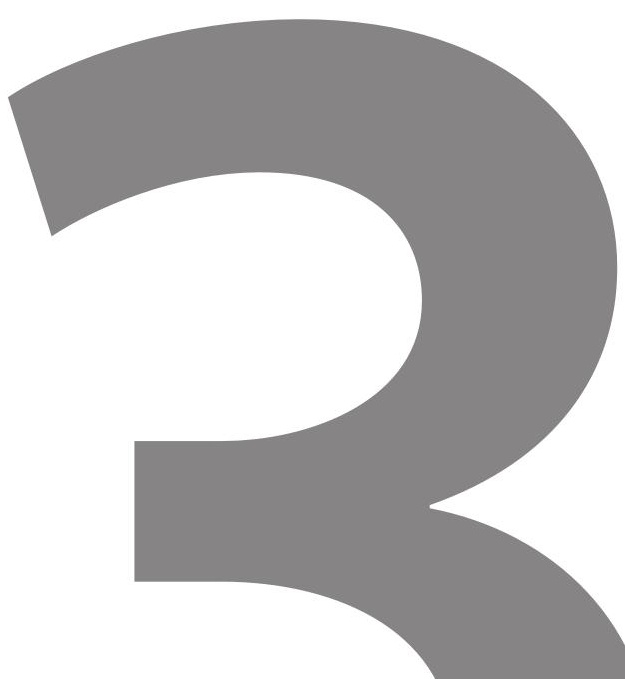




\section{ABSTRACT}

Background. This study examined food desires in daily life, by comparing overweight and healthy-weight participants right before eating events and at non-eating moments. It was hypothesized that overweight participants would have (1) more frequent, (2) stronger, and (3) a greater variety of high-caloric food desires, and (4) consume more high-caloric foods, than healthy-weight participants.

Methods. Ecological momentary assessment (EMA) was used to assess food desire strength and frequency, variety of specific food desires, and food intake. 57 Overweight and 43 healthy-weight adult participants were assessed at eating events and at on average 8 random non-eating moments per day for two weeks. Foods were categorized as: highcaloric highly palatable foods, fruits and salads, staple food dishes and sandwiches, and soups and yoghurts.

Results. Overweight participants reported more frequent high-caloric food desires specifically at non-eating moments than did healthy-weight participants. Healthy-weight participants reported more food desires for staple foods, specifically at eating events. Moreover, overweight participants desired a greater variety of high-caloric foods than healthy-weight participants at both eating events and random non-eating moments. No other betweengroup differences were found.

Conclusions. Results highlighted the importance for obesity interventions (1) to specifically target high-caloric food desires that are experienced during the day and are not tied to eating moments, and (2) to aim for reduction in the variety of high-caloric food desires. This can be accomplished by tailoring interventions to high-caloric food desire in daily life on an individual basis. 


\section{INTRODUCTION}

Many foods have a hedonic quality that can drive food desire and eating behaviour (Lowe \& Butryn, 2007; Pelchat, 2002). Food desires are common (Hill, 2007), and people often desire foods that are high in calories and low in nutritional value (Chao, Grilo, White, \& Sinha, 2014; Hofmann, Adriaanse, Vohs, \& Baumeister, 2013). High-caloric food intake has been associated with weight gain and obesity (Rosenheck, 2008). Furthermore, sensitivity to the rewarding properties (Davis, Strachan, \& Berkson, 2004) and the reinforcing value (Saelens \& Epstein, 1996) of palatable foods is stronger for people with a higher body mass index (BMI) than those with a lower BMI. The goal of the present paper was to investigate food desires and intake of overweight (BMI $\left.\geq 25 \mathrm{~kg} / \mathrm{m}^{2}\right)$ and healthy-weight $\left(18.5 \mathrm{~kg} / \mathrm{m}^{2} \leq \mathrm{BMI}<25 \mathrm{~kg} / \mathrm{m}^{2}\right)$ people in daily life, by means of ecological momentary assessment (EMA).

Previous research on food desire, mainly relying on retrospective self-report (questionnaire) assessment, has found that overweight people have more frequent specific food cravings and desires, mainly for high-caloric foods, than healthy-weight people do (Chao et al., 2014; Delahanty, Meigs, Hayden, Williamson, \& Nathan, 2002; Franken \& Muris, 2005; White et al., 2002). Moreover, priming overweight dieters - as compared to healthy-weight dieters - with tempting high-caloric foods led to higher subsequent desire for high-caloric foods (Ouwehand \& Papies, 2010). So, increased food desire appears to be an important aspect of obesity. However, retrospective self-report assessment is subject to memory recall biases: more recent and more emotionally salient memories are disproportionately often recalled (Schwarz, 2007; Shiffman, Stone \& Hufford, 2008). Investigating food desires as they occur in daily life could lead to more ecologically valid insights.

So far, only few EMA studies with measures of food desire frequency and strength have been conducted. These studies have mainly focused on dieters. Approximately $17 \%$ (Hofmann et al., 2013) to 50\% \% (McKee, Ntoumanis, \& Taylor, 2014) of daily life food desires resulted in dietary lapses. Interestingly, strength and frequency of food desires were not related to dietary restraint (Hofmann et al., 2013), but dieters were more likely to give in to food temptations if the desire to eat was stronger (McKee et al., 2014). It is currently not known how food desires and food intake in daily life differ between overweight and healthy-weight people.

Another aspect of food desire relates to the variety of desired foods. Although previous research has linked food intake variety to obesity (McCrory et al., 1999; Raynor \& Epstein, 2001; Raynor et al., 2005), not much is known about food desire variety. Sensory-specific satiety has been suggested to explain the link between intake variety and obesity (Rolls, 1986; Rolls, 2005): satiety occurs separately for each of the sensory characteristics of different kinds of foods. So, when a large variety of foods is consumed, it will take longer for satiety to set in, which may lead to increased intake (Raynor, Niemeier, \&Wing, 2006). In general, food desires and subsequent intake of these desired foods are highly positively associated (Chao et al., 
2014; Martin, O'Neil, Tollefson, Greenway, \& White, 2008; Weingarten \& Elston, 1991; White et al., 2002), so one possibility is that food desire variety is also related to obesity.

Taken together, the present study investigated how food desire frequency, strength, and variety, and food intake are related to weight status. In addition, the association between specific food desires and food intake is investigated. These questions are addressed by using EMA, a method with high ecological validity. EMA is used to obtain repeated measurements of people's everyday lives (Mehl \& O'Conner, 2013). In the present study, participants were prompted to complete brief questionnaires on their iPhone throughout the day, and prior to eating events. EMA conducted via an electronic device may be especially suitable to assess food desires, because of the relatively short duration of such desires (Berkman, Giuliani, \& Pruitt, 2014).

In sum, this study addresses the following hypotheses: overweight participants (1) report more frequent, and (2) stronger food desires for high-caloric foods, (3) desire a greater variety of high-caloric foods, and (4) consume more high-caloric foods, as compared to healthy-weight participants. Food desire frequency, strength and variety are investigated and compared separately for eating events (that were about to occur) and non-eating moments. There were no specific a priori hypotheses about differences between eating events and non-eating moments in terms of food desire frequency, strength or variety.

\section{METHOD}

The data in the present paper were collected as part of a large ecological momentary assessment (EMA) study investigating predictors of eating behaviour in daily life. A previous paper (Boh et al., 2016) focused on cognitions and emotions. One other previous paper focused on network analysis of associations between food desire, emotions and eating events (Spanakis, Roefs, Boh \& Weiss, 2016). The study was approved by the Ethical Committee of the Faculty of Psychology and Neuroscience of Maastricht University.

\section{Participants}

Inclusion criteria for participation in the study were: (1) BMI between 18.5 and 40, (2) in possession of an iPhone, (3) not on a supervised diet, (4) no medical conditions that could affect eating behaviour, and (5) not pregnant. A total of 67 overweight and 44 healthyweight participants applied. Note that in the present paper, the term 'overweight' also refers to obese $\left(\mathrm{BMI}>30 \mathrm{~kg} / \mathrm{m}^{2}\right)$ participants. Six overweight participants were lost to dropout and five participants ( $n=4$ overweight; $n=1$ healthy-weight) were excluded from participation because of $<10 \%$ compliance with the EMA protocol. Two participants provided a selfreported $\mathrm{BMI}$ measurement at initial screening that was below 40 , but turned out to have a 
BMI of 40.8 and 45.7 upon actual BMI measurement. These participants were included in the final sample to increase overall study power.

The final sample consisted of 57 overweight ( 7 male) and 43 healthy-weight (5 male) participants. There were no significant differences in BMI between the post-EMA measurement (overweight: $M=30.2 ; S D=4.2$; healthy-weight: $M=22.2 ; S D=1.5$ ) and pre-EMA measurement (overweight: $M=30.3 ; S D=4.3$; healthy-weight: $M=22.1 ; S D=1.5$ ) for the overweight group, $t(56)=1.07, p=0.29$, nor for the healthy-weight group, $t(42)=1.22$, $p=0.23$. Furthermore, education level $\left(x^{2}=2.3, p=0.31\right)$ and sex-ratio $\left(x^{2}<0.01, p>0.99\right)$ did not differ between groups. The mean age of participants was $31.2(S D=10.0)$ for the overweight group and 32.1 (SD = 10.6) for the healthy-weight group, with no difference between overweight and healthy-weight participants, $t(98)=0.43, p=0.67$.

\section{General EMA protocol}

Participants used an iPhone application to complete assessments of predictors of eating behaviour (food desires, emotions, cognitions preceding food intake, physical locations, and activities) and food intake. This paper focused on measures of desires and intake. Food desire was assessed both immediately prior to each self-reported eating event, and after receiving an automated notification on the participant's iPhone requesting an assessment (non-eating moment assessments). Non-eating moment assessments were randomly distributed over the waking day, occurring once per 2-hour time window. Sleeping and waking times were modifiable in the app.

\section{Measures of food desire and intake}

\section{At eating events and non-eating moments}

At each assessment, strength of food desire was indicated on a visual analogue scale (VAS) ranging from 0 to 100 by answering the question: "How strong is your desire to eat?". Note that in the Dutch language, there is no clear distinction between 'food desire' and 'food craving. Importantly, on each occasion, the participant was asked whether (s)he desired (a) specific type(s) of food(s). So, food desires could be either for specific foods or unspecified. If (s)he answered "yes", an overview of 19 food-icons was presented, reflecting different types of foods. See Table 1. These 19 food types were chosen to reflect the most common food types eaten in a typical Dutch diet. The participant was instructed to select icons of food(s) that most closely resembled the food(s) (s)he desired. Multiple selections were possible. For example, if a participant desired salty sticks, (s)he was instructed to select the icon 'chips' (i.e., representing the category 'salty snacks'). Participants received printed manuals of the app functionality. 


\section{At eating events only}

At eating events, an additional question, "What are you about to eat?", was asked, and the participant was instructed to indicate what (s)he was about to eat via the same overview of 19 food-icons used for the food desire question. Multiple food-type selections were possible. To verify that food intake took place, participants were asked to take a picture of the food. Note that food quantity was not measured.

\section{Procedure}

Prior to starting, the participant followed a one-day training to get familiar with the app and EMA procedure, and to resolve technical issues. In addition, the participant was instructed to obtain measurements of body weight and height. These measurements were conducted by healthcare professionals or a researcher for $89 \%$ of participants (11\% self-reported height and weight). After obtaining these measurements, the participant was enrolled in a 2-week EMA period, in which (s)he reported on predictors of eating behaviour and food intake. After the EMA period, the participant was asked to have body weight measured again under identical circumstances as for the first measurement. After obtaining the measurement, the participant was debriefed about the purposes of the study and received $a € 50$,- voucher.

\section{Data analysis}

The 19 food types were first sorted into four main categories: (1) high-caloric, highly palatable foods (HCHP), (2) fruits and salads, (3) sandwiches and foods often served as staple parts of a dinner (staple), and (4) soups and yoghurts. Table 1 depicts to what category each food type was allocated. All analyses were performed in SPSS v. 23.0 (SPSS Inc., USA) and were conducted using two-tailed independent samples t-tests, comparing healthy-weight with overweight participants. For these t-tests, a negative difference score means that overweight participants scored higher than did healthy-weight participants, whereas a positive difference score means that overweight participants scored lower than did healthy-weight participants.

For each participant, the total number of specific food desires was summed separately for eating events and non-eating moments, over the entire 2-week period. These numbers were then averaged within each participant group (overweight and healthy-weight). To

Table 1. Food icons as allocated to each food type category.

Food type category
$\begin{gathered}\text { Represented by these icon(s) } \\ \text { High-caloric highly palatable } \\ \text { Fruits and salads } \\ \text { Staple dishes and sandwiches } \\ \text { with a side of fries, Chips, Pizza, Cake, Ice cream } \\ \text { Soups and yoghurts }\end{gathered} \quad \begin{gathered}\text { Salad, Apple } \\ \text { Sandwiches, Pasta, Cornflakes, Dishes with a side of } \\ \text { potatoes, Dishes with a side of rice }\end{gathered}$
Yoghurt, Soup


determine how often each category of foods was desired, percentages were computed per food category, relative to the total number of specific food desires for each participant, summed over all eating events and non-eating moments combined. These percentages were then averaged over participants within each participant group.

Desire strength was only scored once during each EMA assessment. In case of multiple food desires, this desire strength score was considered to apply equally to all selections for that particular assessment. For each participant, ten desire strength scores were computed, by averaging desire strength scores of the four food categories and for non-specific desires, separately for eating events and non-eating moments. These were then averaged within each participant group.

It was also determined for each participant how often a food from each of the four categories was eaten during the two-week period, by computing percentages relative to the total number of food products that were eaten by each participant. These percentages were then averaged within each participant group.

Lastly, variety of food desire and food intake was determined. To increase comparability between the number of foods in the HCHP category (which concerns ten food-types) and in the other three categories (which concern two, five and two food-types respectively), data of these latter three categories were summed (referred to as 'other foods'). For each participant, and separately for each food category (HCHP or 'other foods'), the number of different food desires and the number of different consumed foods during the 2-week EMA period were determined. These numbers were then divided by the total number of foods per category and multiplied by 100 . This provided a percentage, where $100 \%$ indicates all foods of a category were desired for or consumed. These percentages were then averaged within each participant group. One participant was excluded from the food desire variety analyses because (s)he did not report any specific food desires.

\section{RESULTS}

\section{Compliance}

The percentage of completed relative to the total number of non-eating moment assessment requests participants received during the 2-week EMA period was calculated. Compliance did not differ significantly between the two groups, $t(98)=0.59, p=0.55,95 \%$ $\mathrm{Cl}=[-5.14,2.77]$ (overweight: $80.91 \%, \mathrm{SD}=9.96 \%$, range $=[50.89,96.43]$; healthy-weight: $79.73 \%, S D=9.73 \%$, range $=[50.00,95.54])$. Overweight participants did not report more eating events than healthy-weight participants, $t(98)=1.59, p=0.12,95 \% \mathrm{Cl}=[-0.10,0.91]$ (eating events per day: overweight: $M=3.77, S D=1.05$; healthy-weight: $M=4.17, S D=1.50$ ). Note that at each eating event, multiple food types could be selected. 


\section{Overall frequency of desires for specific foods}

Desires for specific foods were reported on average for $40.68 \%$ (SD $=30.43 \%$ ) of all eating events for overweight participants and for 38.88\% (SD $=28.83 \%$ ) of all eating events for healthy-weight participants, with no significant difference between groups, $t=0.30$, $p=0.77,95 \% \mathrm{Cl}=[-13,73,10.13]$. For the remainder of the eating events, no specific food desire was reported. On average, $14.23 \%$ (SD $=11.92 \%$ ) of non-eating moments of overweight participants, and $14.24 \%$ (SD $=13.36 \%)$ of non-eating moments of healthy-weight participants contained a desire for specific foods, with no significant difference between groups, $t=0.004, p>0.99,95 \% \mathrm{Cl}=[-5.02,5.04]$.

There was no difference between the average total number of reported desires during the 2-week period for specific foods reported between healthy-weight $(M=22.1$; $S D=19.51$ ) and overweight $(M=22.3 ; S D=18.6)$ participants at eating events, $t(98)=0.05, p=0.96,95 \%$ $C l=[-7.84,7.50]$, nor at non-eating moments (overweight: $M=13.05 ; S D=11.77$; healthyweight: $M=14.02 ; S D=12.06), t(98)=0.40, p=0.69,95 \% \mathrm{Cl}=[-3.81,5.76]$.

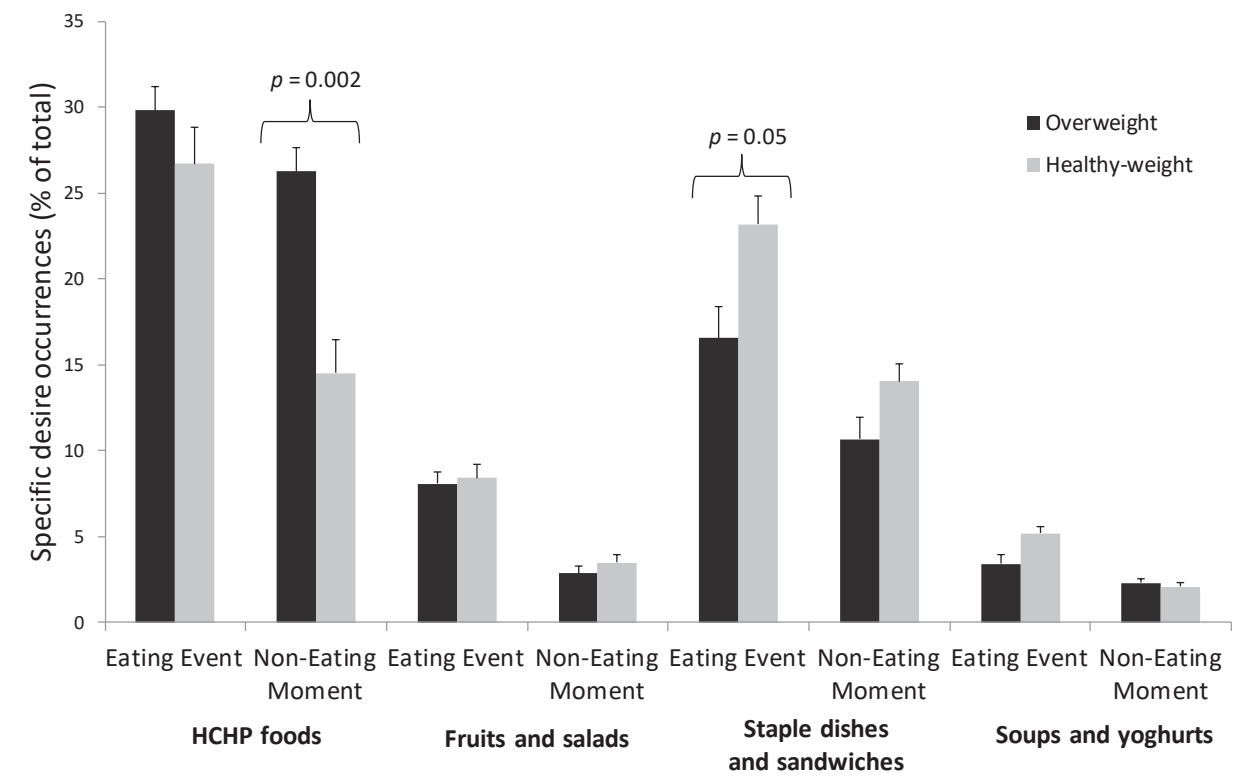

Figure 1. Average specific desire occurrences of overweight and healthy-weight participants.

Note. Data are presented per food type (high-caloric highly palatable (HCHP) foods, fruits and salads, staple dishes and sandwiches and soups and yoghurts). Percentages were first computed relative to the total number of specific food desires for each participant individually, summed over all eating events and non-eating moments combined. These percentages were then averaged for the overweight and healthy-weight groups. Error bars represent 1 standard error of the mean. One overweight participant did not report any specific food desire occurrences and was not included in the analyses. 


\section{Frequency of desires for specific foods, per food category}

Food categories were: (1) HCHP foods, (2) fruits and salads, (3) staple foods, and (4) soups and yoghurts. Overweight participants reported significantly more specific HCHP food desires at non-eating moments than healthy-weight participants, $t(98)=-3.21, p=0.002,95 \% \mathrm{Cl}$ $=[-19.03,-4.49]$. In addition, healthy-weight participants reported trend-significantly more staple food desires at eating events than overweight participants, $t(98)=1.97, p=0.052$, $95 \% \mathrm{Cl}=[-0.05,13.31]$. None of the other comparisons reached significance, all $t<1.51$, all $p>0.13$. See Figure 1.

\section{Food desire strength scores}

Desire strength scores were analysed separately for each of the four food categories and for non-specific desires. None of the between-group comparisons reached significance, all $t<1.77$, all $p>0.08$. One trend was observed, in that healthy-weight participants tended to report higher non-specific desire strength scores at non-eating moments. See Table 2.

Table 2. Desire strength scores and comparisons between overweight and healthy-weight participants for each food category and for non-eating moments.

\begin{tabular}{|c|c|c|c|c|c|c|c|c|c|}
\hline & \multirow[t]{2}{*}{$\begin{array}{c}\text { Score categories for } \\
\text { food desires }\end{array}$} & \multicolumn{2}{|c|}{$\begin{array}{c}\text { Overweight } \\
\text { participant scores }\end{array}$} & \multicolumn{2}{|c|}{$\begin{array}{l}\text { Healthy-weight } \\
\text { participant scores }\end{array}$} & \multicolumn{4}{|c|}{ Comparisons } \\
\hline & & $M$ & SD & $\mathbf{M}$ & SD & t & df & p & $95 \% \mathrm{Cl}$ \\
\hline \multirow[t]{5}{*}{ Eating events } & Nonspecific & 53.74 & 16.16 & 54.92 & 16.93 & 0.36 & 97 & 0.72 & {$[-5.45,7.83]$} \\
\hline & $\mathrm{HCHP}$ & 59.37 & 17.66 & 60.64 & 16.20 & -0.36 & 91 & 0.72 & {$[-8.36,5.82]$} \\
\hline & Fruits and vegetables & 58.30 & 18.72 & 57.35 & 17.68 & 0.21 & 64 & 0.83 & {$[-8.03,9.94]$} \\
\hline & Staple foods & 69.64 & 13.91 & 71.15 & 12.54 & -0.50 & 76 & 0.62 & {$[-7.54,4.53]$} \\
\hline & Soups and yoghurts & 61.04 & 20.67 & 64.09 & 17.65 & -0.56 & 49 & 0.58 & {$[-13.94,7.83]$} \\
\hline \multirow{5}{*}{$\begin{array}{l}\text { Non-eating } \\
\text { moments }\end{array}$} & Nonspecific & 24.48 & 11.57 & 28.57 & 11.21 & 1.77 & 98 & $0.08 \wedge$ & {$[-0.49,8.66]$} \\
\hline & $\mathrm{HCHP}$ & 53.74 & 19.79 & 57.68 & 19.17 & -0.94 & 87 & 0.35 & {$[-12.29,4.42]$} \\
\hline & Fruits and vegetables & 57.09 & 22.29 & 54.12 & 23.22 & 0.44 & 43 & 0.66 & {$[-10.71,16.65]$} \\
\hline & Staple foods & 64.05 & 19.72 & 67.44 & 18.86 & -0.75 & 73 & 0.46 & {$[-12.35,5.59]$} \\
\hline & Soups and yoghurts & 57.02 & 26.87 & 62.26 & 21.22 & -0.69 & 39 & 0.50 & {$[-20.71,10.24]$} \\
\hline
\end{tabular}

$\mathrm{M}=$ Mean; $\mathrm{SD}=$ Standard Deviation.

Note. Desire strength was scored on a VAS item ranging from 0 to 100. Confidence intervals were calculated over the difference. A negative difference score means that overweight participants scored higher than did healthy-weight participants, whereas a positive difference score means that overweight participants scored lower than did healthy-weight participants. Variation in df is due to a varying number of participants not having any eating events / non-eating moments for certain categories.

${ }^{*}=p<0.05 ; \wedge=0.05 \leq p<0.1$.

\section{Foods consumed at eating events}

The frequency of food intake for each of the four food categories was analysed. None of the between-group comparisons reached significance, all $t<1.08$, all $p>0.28$. See Table 3 . 
Table 3. Percentages of food intake per category relative to the total number of consumed foods and comparisons of overweight and healthy-weight participants for each food category.

\begin{tabular}{|c|c|c|c|c|c|}
\hline \multirow[b]{3}{*}{ Food categories } & \multicolumn{2}{|c|}{$\%$ Intake per category } & \multirow{2}{*}{\multicolumn{3}{|c|}{$\begin{array}{c}\text { Comparisons } \\
\mathrm{df}=98\end{array}$}} \\
\hline & Overweight & Healthy-weight & & & \\
\hline & M(SD) & M(SD) & $\mathbf{t}$ & $\mathbf{p}$ & $95 \% \mathrm{Cl}$ \\
\hline High-caloric highly palatable & $25.5(13.22)$ & 23.46(11.39) & -0.81 & 0.42 & {$[-7.04,2.95]$} \\
\hline Fruits and salads & 16.18(10.71) & $16.03(11.54)$ & -0.06 & 0.95 & {$[-4.58,4.29]$} \\
\hline Staple dishes and sandwiches & 49.7(12.4) & $50.38(13.44)$ & 0.26 & 0.79 & {$[-4.47,5.84]$} \\
\hline Soups and yoghurts & $8.62(5.95)$ & 10.13(8.15) & 1.07 & 0.29 & {$[-1.29,4.30]$} \\
\hline Total \% & 100 & 100 & & & \\
\hline
\end{tabular}

$\mathrm{M}=$ Mean; $\mathrm{SD}=$ Standard Deviation.

Note. A negative difference score means that overweight participants scored higher than did healthy-weight participants, whereas a positive difference score means that overweight participants scored lower than did healthy-weight participants.
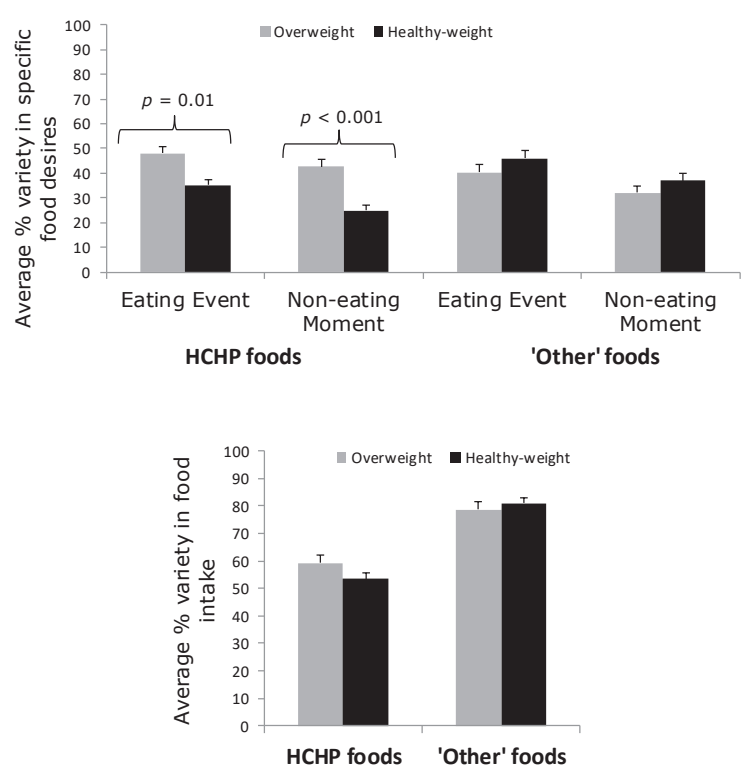

Figure 2. Note. Variety of food desires (a) and variety of food intake (b) were computed by dividing the number of different desired-for and consumed foods respectively by the total number of foods available within each category for each participant (multiplied by 100 to arrive at a percentage). For food desire variety, this was done separately for eating events and non-eating moments. The 'other foods' category is a combination of the three non-HCHP food categories. The total number of available foods was identical for food desires and food intake. The HCHP food category consists of 10 different food-types, whereas the 'Other' foods category consists of 9 different food-types. The error bars represent the standard errors of the mean. One overweight participant did not report any specific food desire occurrences and was not included in the analyses of food desire variety.

\section{Variety in food desires and food intake}

Overweight participants reported significantly more variety in HCHP food desires at eating events, $t(97)=-2.59, p=0.01,95 \% \mathrm{Cl}=[-22.65,-3.02]$, and at non-eating moments, $t(97)=$ $-3.65, p<0.001,95 \% \mathrm{Cl}=[-27.60,-8.24]$, than healthy-weight participants, whereas for the 
'other foods' category, neither the comparison for eating events, nor the comparison for non-eating events reached significance, both $t<0.95$, both $p>0.32$. Variety of food intake did not differ between healthy-weight and overweight participants, for both high-caloric foods, $t(98)=-1.32, p=0.19,95 \% C l=[-14.53,2.91]$, and 'other foods', $t(98)=0.81, p=0.42$, $95 \% \mathrm{Cl}=[-3.48,8.24]$. See Figure 2 .

\section{Matches between specific food desires and eating}

At eating event assessments, specific food desires and eating were considered a match if at least one of the desired-for foods was also consumed. Thus, the number of matches between food desires and intake was determined per eating event, not per food icon selection. For $88.73 \%$ (SD $=21.49 \%$ ) of total specific food desires that were reported at an eating event assessment, there was at least one such match. There were no differences between overweight ( $M=88.1 \%, S D=23.84 \%)$ and healthy-weight $(M=89.23 \%, S D=19.75 \%)$ participants, $t(98)=0.006, p>0.99,95 \% \mathrm{Cl}=[-7.35,7.39]$.

\section{DISCUSSION}

In this study, several aspects of food desires were compared between overweight and healthy-weight participants. The main findings include: (1) overweight participants reported more specific high-caloric (HCHP) food desires at non-eating moments than did healthy-weight participants, whereas healthy-weight participants reported more specific desires for meal-type foods and sandwiches (staple foods) at eating events than did overweight participants. (2) A strong association was observed between the type of food that was desired and that was actually eaten at eating events. This association was not different between overweight and healthy-weight participants. (3) Overweight participants reported a wider variety of specific HCHP food desires than healthy-weight participants, both at eating events and at non-eating moments. No between-group differences were observed for variety in specific food desires for other foods. In addition, no between-group differences were observed for variety in food intake. (4) No differences between overweight and healthy-weight participants were observed for self-reported actual food intake and food desire strength.

Overweight participants reported more frequent desires specifically for HCHP foods, specifically at non-eating moments. In the Western obesogenic environment (Swinburn et al., 2011), food is widely available, so a wide variety of cues can be conditioned to trigger desires for food, such as recurring contexts and activities (Bongers, van den Akker, Havermans, \& Jansen, 2015; Jansen, Schyns, Bongers, \& van den Akker, 2016; Van Den Akker, Havermans, \& Jansen, 2015; van den Akker, Jansen, Frentz, \& Havermans, 2013). Possibly, overweight participants associated more abstract aspects of the environment with the intake of high- 
caloric, rewarding foods than healthy-weight participants, leading to more frequent highcaloric food desires in between meals. On the other hand, food desires of healthy-weight participants were more often tied to eating events such as breakfast, lunch and dinner, and food desire occurred mostly for foods that are typically consumed at those eating events. These findings further expand previous research that showed overweight people have an increased desire for (high-caloric) food (Chao et al., 2014; Delahanty et al., 2002; Franken \& Muris, 2005; White et al., 2002). Such increased desire seems to occur mostly for high-caloric foods, and in-between regular meal times. Note, that these findings were not paralleled in analyses of the strength of food desires.

The association of food desire and subsequent eating in the present study was strong, and similar for overweight and healthy-weight participants: almost 9 in 10 times on average, desire for specific foods at eating moments was followed by intake of at least one type of food that was desired. This finding is in line with findings of a previous laboratory study of overweight participants, in which food desires for certain types of high-caloric foods resulted in significantly more intake of that type of food than of other types of foods (Martin et al., 2008). It is also in line with findings of a questionnaire study that reported that desire for foods with certain sensory aspects (e.g., sweetness) was followed by intake of foods with those specific sensory aspects, irrespective of participants'BMI (Chao et al., 2014). This strong connection is likely due to the timing of the measurement, in that participants were about to eat and the food was in front of them.

In line with the hypothesis, overweight participants reported more varied HCHP food desires than healthy-weight participants, at eating events and at non-eating moments. So, it seems that the more frequent occurrence of specific HCHP food desires for overweight participants does not reflect desire for particular HCHP foods (e.g., chocolate lovers), but likely reflects desire for many different HCHP foods. For food intake variety, however, contrary to previous research (McCrory et al., 1999; Raynor \& Epstein, 2001; Raynor et al., 2005), no between-group difference was observed. It should be noted that, in the present study, food intake was often not accompanied by a specific food desire. That is, specific food desires occurred only for about $40 \%$ of the eating events, which may have reduced the likelihood of finding group differences.

In the present study, there were no overall differences in frequency of eating events between overweight and healthy-weight participants. This could indicate a selective underreporting of eating events in the overweight group. Previous research found that underreporting of food intake was associated with BMI (Heerstrass, Ocké, Bueno-De-Mesquita, Peeters, \& Seidell, 1998; Kretsch, Fong, \& Green, 1999). Overweight participants may have been more conscious about their food intake during the 2-week EMA period, leading to more intake control efforts. In addition, as with questionnaire-based research in general, social desirability could have influenced the reporting of eating events (Heerstrass et al., 1998). Lastly, food quantity was not assessed in the present study. Although the number of 
reported eating events in the present study is comparable to other EMA research on food intake (Tomiyama, Mann \& Comer, 2009), it is possible that overweight and healthy-weight people differ in the amount of food consumed per eating event.

Concluding, EMA of eating events and non-eating moments enabled on-line insights into the specificity, strength and variety of food desires and how these relate to food intake. This would not have been possible with the use of retrospective measures. As compared to healthy-weight participants, overweight participants' high-caloric food desires more commonly occurred throughout the day, not tied to self-reported eating events. Healthy-weight participants more often reported staple food desires when about to eat, as compared to overweight participants. Moreover, both at eating events and otherwise throughout the day, food desires were more varied for overweight than for healthy-weight participants. So, the present findings suggest that an important focus for obesity treatment is (1) to reduce high-caloric food desires that are not tied to the usual mealtimes, and (2) to reduce variety of high-caloric food desires. By learning to control and inhibit urges to eat high-caloric foods, further weight gain may be prevented and weight may be lost (Houben, 2011). The present results suggest that tailoring to individual high-caloric food desires via EMA is feasible. By doing so, ecological momentary interventions could provide therapy directly after a highcaloric food desire is reported. 

PART 2

Cognitive behavioral therapybased ecological momentary intervention for obesity 



\section{Chapter 4}

\section{An ecological momentary}

intervention for weight loss and

healthy eating via smartphone

and internet: Study protocol for a

randomised controlled trial

B. Boh

L. H. J. M. Lemmens
A. Jansen
C. Nederkoorn
V. Kerkhofs
G. Spanakis
G. Weiss
A. Roefs

(2016). An ecological momentary intervention for weight loss and healthy eating via

smartphone and internet: study protocol for a randomised controlled trial.

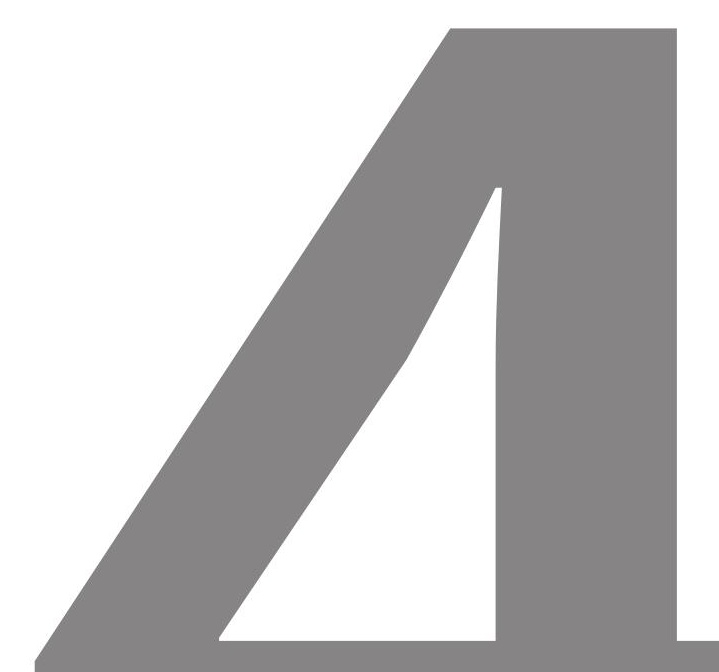




\section{ABSTRACT}

Background: Long-term weight loss maintenance is difficult to achieve. Effectiveness of obesity interventions could be increased by providing extended treatment, and by focusing on person-environment interactions. Ecological Momentary Intervention (EMI) can account for these two factors by allowing an indefinite extension of a treatment protocol in everyday life. EMI relies on observations in daily life to intervene by providing appropriate in-themoment treatment. The Think Slim intervention is an EMI based on the principles of Cognitive Behavioural Therapy (CBT), and its effectiveness will be investigated in the current study.

Methods: A randomised controlled trial (RCT) will be conducted. At least 134 overweight adults (Body Mass Index (BMI) $>25 \mathrm{~kg} / \mathrm{m}^{2}$ ) will be randomly assigned to an 8-week immediate intervention group (Diet + Think Slim intervention, $n=67$ ) or to an 8-week diet-only control group (followed by the Think Slim intervention, $n=67$ ). The Think Slim intervention consists of (1) an app-based EMI that estimates and intervenes when people will likely overeat, based on ecological momentary assessment data, and (2) ten online computerized CBT sessions which work in conjunction with an EMI module in the app. The primary outcome is BMI. Secondary outcomes include (1) scores on self-report questionnaires for dysfunctional thinking, eating styles, eating disorder pathology, general psychological symptomatology, and self-esteem, and (2) eating patterns, investigated via network analysis. Primary and secondary outcomes will be obtained at pre- and post-intervention measurements, and at 3 and 12 month follow-up measurements.

Discussion: This is the first EMI aimed at treating obesity via a cognitive approach, provided via a smartphone app and the internet, in the context of an RCT.

Trial registration: This trial has been registered at the Netherlands Trial Register, part of the Dutch Cochrane Centre (NTR5473; registration date: 26 ${ }^{\text {th }}$ of October, 2015; http://www. trialregister.nl/trialreg/admin/rctview.asp?TC $=5473$ ). 


\section{BACKGROUND}

Obesity is so prevalent that it is considered a pandemic (Swinburn et al., 2011). There are many adverse health effects associated with obesity, such as increased rates of type-2 diabetes, cardiovascular disease, osteoarthritis, obesity-related cancers, and psychological disorders such as depression (Dixon, 2010). As a consequence, in the U.S., obesity has been associated with an annual medical care cost of $\$ 209.7$ billion, corresponding with $20.6 \%$ of total annual spending (Cawley \& Meyerhoefer, 2012). In the UK, obesity-related diseases are estimated to add £1.9-2 billion per year to healthcare costs by the year 2030 (Wang, McPherson, Marsh, Gortmaker, \& Brown, 2011).

So far, behavioural obesity treatment has been largely ineffective at achieving sustained maintenance of weight loss that is required to curb the obesity pandemic (Brownell, 2010). Whereas some degree of initial weight loss is often achieved, it is almost invariably followed by weight regain. Meta-analyses on weight maintenance have shown that diet programs with or without exercise (Anderson, Konz, Frederich, \& Wood, 2001; Wu, Gao, Chen, \& van Dam, 2009), very-low-energy diets (Anderson et al., 2001; Franz et al., 2007), dietary counselling (Dansinger, Tatsioni, Wong, Chung, \& Balk, 2007), behavioural therapy (Shaw, O'Rourke, Del Mar, \& Kenardy, 2006), lifestyle advice (Douketis, Macie, Thabane, \& Williamson, 2005) and pharmacotherapy (Douketis et al., 2005; Franz et al., 2007; Haddock, Poston, Dill, Foreyt, \& Ericsson, 2002) mostly lead to weight loss between 5-9\% of initial body weight, which levels off at around six months (Franz et al., 2007). After these six months, around 50\% of the lost weight (on average) is gradually regained (Curioni \& Lourenço, 2005; Franz et al., 2007). This indicates that it is important to investigate methods for improving not only immediate weight loss resulting from treatment, but also to prevent weight regain in the long term.

\section{Increasing the effectiveness of obesity treatment}

Two promising factors could increase the effectiveness of behavioural obesity treatment in the long term. Firstly, the high prevalence of weight regain implies that obesity should be considered a chronic condition that requires continuous care (Bray, 2004; Foreyt, Carlos, Poston, \& John, 1998; Fujioka, 2002; Mietus-Snyder \& Lustig, 2008; Perri, Sears, \& Clark, 1993; Rippe, Crossley, \& Ringer, 1998). It has been shown that providing extended care post-obesity treatment (in the form of post-treatment contact sessions with an interventionist) leads to better long-term weight loss maintenance (Middleton, Patidar, \& Perri, 2012). Observations from the National Weight Control Registry furthermore show that people who successfully maintain long-term weight loss continue to consistently monitor their eating behaviour and body weight, engage in physical exercise, and limit caloric intake post-weight loss (Ogden et al., 2012; Wing \& Phelan, 2005). Secondly, an effective treatment model for obesity should account for interactions between individual (psychological) factors and the food-replete obesogenic environment (Blakemore \& Froguel, 2008; Carter \& Jansen, 2012; Egger \& Swin- 
burn, 1997; Hill \& Peters, 1998; Lang \& Froelicher, 2006; Perri et al., 1993; Swinburn, Egger, \& Raza, 1999; Swinburn et al., 2011).

\section{Ecological Momentary Interventions}

With the advent of mobile technology and the internet, it has become possible to provide continuous care that can account for person-environment interactions. Ecological momentary intervention (EMI) is a framework that combines real-time assessment (Ecological Momentary Assessment, EMA) with treatment. Therefore, EMI allows the provision of (indefinite) care in the natural environment (Heron \& Smyth, 2010). To accomplish this, assessment and treatment is conducted and provided via a mobile platform, such as a smartphone. The advantage over traditional treatment is that EMI does not necessarily involve therapist contact. This makes treatment more accessible for people that feel reluctant to seek help within a traditional healthcare system. Furthermore, EMI is associated with lower costs (Heron \& Smyth, 2010). Instead of extensive therapist contact, EMI uses observations of daily life, via for example a smartphone, as input to automatically guide therapy-based techniques and progress. Therefore, EMI is most suitable in combination with a well-defined and structured intervention protocol, such as cognitive behavioural therapy (CBT), which can more easily be automated. So far, only one pilot study has been conducted investigating EMI for obesity, which was aimed at changing the eating behaviour of overweight participants (Atienza, King, Oliveira, Ahn, \& Gardner, 2008). This study showed that participants' intake of healthful food increased at the end of the intervention, and that participants considered the intervention acceptable (Atienza et al., 2008). However, the study relied on providing feedback on EMA data and did not include a treatment protocol. Therefore, more research is necessary to improve insights into the effectiveness of EMI for obesity.

\section{Think Slim Intervention}

The Think Slim intervention is designed to work in conjunction with a calorie-restrictive diet. It consists of (1) an iPhone app-based EMI that will estimate when people will be likely to overeat (risky moments) and that can intervene at such moments, and (2) a CBT-based intervention aimed at changing patterns of obesity-related dysfunctional thinking and increasing self-esteem. The CBT-based intervention consists of ten online sessions on a computer (computerized CBT; CCBT), and a module in the app. The CBT app module will allow participants to make use of cognitive techniques and reflection practised during the online sessions.

The EMI part of the intervention uses observations in daily life that are obtained via EMA to build a dynamic network of interactions (over time) between psychological variables (Borsboom \& Cramer, 2013; Bringmann et al., 2013; Wichers, 2013). Specifically, variables with relevance for eating behaviour were included, such as mood ( $\mathrm{O}^{\prime} \mathrm{Connor}$, Jones, Conner, Mcmillan, \& Ferguson, 2008), physical location and activities (Patel \& Schlundt, 2001), food 
desire strength and specific food desires (Chao, Grilo, White, \& Sinha, 2014). Previous research has shown that specific patterns in such a network (i.e., recurring contingencies between variables) can be associated with psychological disturbances (Wichers, 2013). For obese participants, contingencies between EMA-assessed eating-related variables modelled in a network were previously found to be more often and more strongly present, leading to a denser network structure as compared to non-obese participants (Spanakis et al., 2015). The Think Slim intervention uses contingencies between variables of observations collected in daily life to estimate 'risky' moments that indicate when it is likely that someone will overeat. So, by considering network patterns that consistently precede overeating, treatment in the app, in the form of a warning and CCBT-based feedback, can occur prior to an actual eating event.

In addition to estimating risky moments, insights into how variables such as mood states, physical locations, and activities are related to eating behaviour will be provided as summarized graphical feedback that is accessible in the iPhone app. Although no data exists for treating obesity, one previous randomized controlled trial found this sort of graphical feedback to be beneficial for treating depression (Kramer et al., 2014).

The second part of the Think Slim intervention is based on the principles of CBT (e.g., Beck, 2011). The cognitive theory states that negative emotions and undesirable behaviour are caused and maintained by maladaptive information processing and dysfunctional beliefs. To decrease psychopathology, CBT focuses on identifying and altering the function, content and structure of cognitions, schemas and attitudes associated with undesirable behaviour. Over the course of CBT treatment, patients are guided through several structured sessions aimed at identifying and modifying dysfunctional thinking patterns. Patients are asked to monitor their cognitions and learn to evaluate the validity and utility of these cognitions. Furthermore, they gradually replace unrealistic cognitions with more helpful alternatives.

CBT is one of the most thoroughly supported psychological interventions worldwide (Hollon \& Beck, 1994) and was found to be effective for a wide range of psychological disorders including for example anxiety and depression (Butler, Chapman, Forman, \& Beck, 2006). Though research in the field of obesity is scarce, it has been suggested that CBT might be an effective treatment for obesity as well (Carter \& Jansen, 2012; Cooper \& Fairburn, 2001). Various trials have found support for long-term weight loss maintenance in obese participants (Stahre \& Hällström, 2005; Stahre, Tärnell, Håkanson, \& Hällström, 2007; Werrij et al., 2009). However, one trial did not (Cooper et al., 2010). Furthermore, CBT was found to be effective in reducing dysfunctional cognitions in morbidly obese patients waiting to undergo bariatric surgery (Ashton, Drerup, Windover, \& Heinberg, 2009; Gade, Hjelmesæth, Rosenvinge, \& Friborg, 2014), and in establishing long-term weight loss in obese participants with binge eating disorder (Vanderlinden et al., 2012).

In the current study, CBT will be provided via online sessions on a computer (CCBT), and via a module in the app. So far, not much is known about the efficacy of CCBT for the treatment 
of obesity. However, a recent meta-review concluded that CCBT is effective in the treatment of depression (Foroushani, Schneider, \& Assareh, 2011). Furthermore, research indicates that CCBT is considered an acceptable intervention by clients (de Graaf, Huibers, Riper, Gerhards, \& Arntz, 2009; Kaltenthaler et al., 2008). There is also pilot study data indicating CBT delivered via an app can be as effective as CCBT, with both interventions resulting in a significant decline in depressive symptomatology (Watts et al., 2013).

\section{Aims and hypotheses}

The current study aims to test the effectiveness of the Think Slim intervention. In a randomized controlled trial, overweight participants will actively follow a diet of their own choice. In addition, they will be instructed to exercise according to their own personal exercise plan. Half of the participants will receive the Think Slim intervention immediately (immediate intervention group). The other half will start with a diet only (diet-only control group). Participants in this latter group will receive the Think Slim intervention afterwards. It will be examined whether diet + Think Slim is superior to diet-only in the reduction of Body Mass Index (BMI) and on a set of secondary outcomes at post-intervention. Secondary outcomes include (1) self-report questionnaires of dysfunctional thinking, eating styles, general psychological distress, eating disorder pathology, and self-esteem, and (2) network patterns that lead to unhealthy eating. It is expected that over the course of receiving the Think Slim intervention, participants in the immediate intervention group will experience (1) greater reduction in $\mathrm{BMI}$, (2) greater improvements on dysfunctional thinking, self-report questionnaires of eating styles, eating disorder pathology, general psychological symptomatology, and self-esteem, and (3) have less dense network connectivity related to unhealthy eating, as compared to participants in the diet-only control group. Lastly, it is expected that, for both groups taken together, reductions in BMI and improvements on secondary measures will have been maintained at the 3 and 12 month follow-up measurements.

\section{METHODS}

\section{Trial design}

A randomised controlled superiority trial will be conducted, in which 134 overweight (BMI $>25 \mathrm{~kg} / \mathrm{m}^{2}$ ) participants will follow a diet of their own choice. In addition, they will exercise according to their own personal exercise plan. Participants are split into two groups. The two groups will run in parallel. The immediate intervention group $(n=67)$ will immediately receive the Think Slim intervention in addition to the chosen diet. During this time, the dietonly control condition group $(n=67)$ will receive no further support. The diet-only control condition group will receive the Think Slim intervention after the immediate intervention group has completed the intervention. The enrolment procedure is graphically shown in 
Figure 1. The trial has been approved by the Ethics Committee of the Faculty of Psychology and Neuroscience of Maastricht University (the Netherlands), and is registered at the Dutch Trial Register, part of the Dutch Cochrane Centre (NTR5473). The study will adhere to recommendations of the Consolidated Standards of Reporting Trials (CONSORT) (Consort statement website, accessed April 2015).

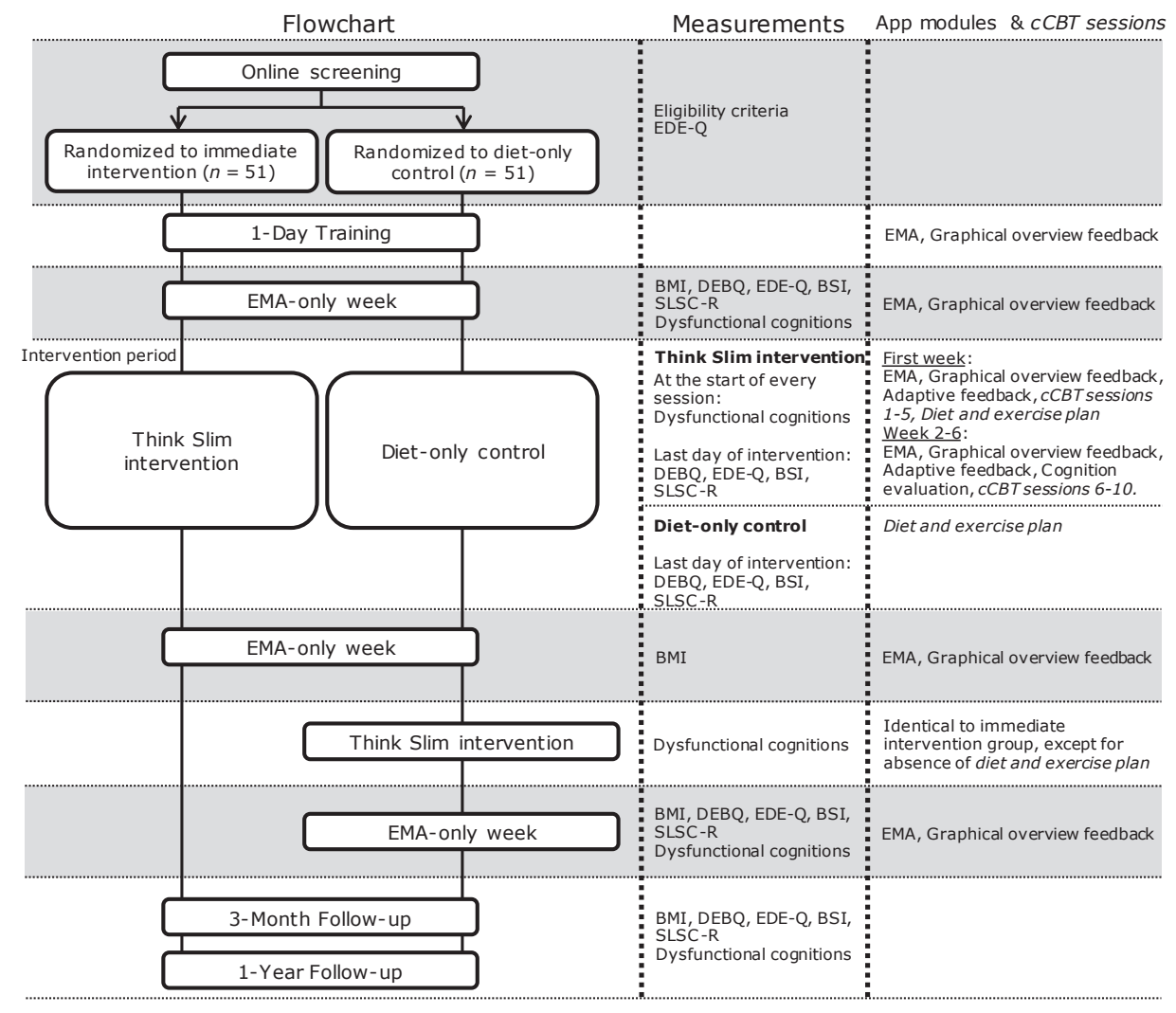

Figure 1. Flowchart for the immediate intervention and diet-only control groups.

Note. Timing for the 3-month and 1-year follow-up measurements will be calculated from the end of the last EMA-only week for both groups.

\section{Participants}

A sample of 134 overweight adults who are recruited in the general Dutch and Belgian population will be included. Applicants will be eligible to participate if they meet the following criteria: age between 18 and 60 years old, Body Mass Index (BMI) $>25 \mathrm{~kg} / \mathrm{m}^{2}$, sufficient knowledge of the Dutch language, and in possession of an iPhone. Exclusion criteria are: the presence of an eating disorder (as determined with the Eating Disorder Examination Questionnaire, EDE-Q, Fairburn \& Beglin, 1994), the use of appetite-influencing medication 
(e.g., Suboxone), current treatment for depression, enrolment in other obesity treatments, pregnancy, and a diagnosis of diabetes-2.

\section{Sample size}

Sample size was calculated a-priori using G*Power (Faul et al., 2007). Prior effect size estimates are not available because the current study will be the first to investigate an EMI for weight loss. Power analysis indicated that with 51 participants in each condition, the study is powered at $80.6 \%$ (one-tailed, $a=0.05$ ) to detect a standardized medium effect size difference (Cohen's $d=0.5$; Cohen, 1992) in BMI change between the two conditions. To account for an expected $23.5 \%$ attrition rate (derived from attrition rates in EMI studies with ten or more participants, reported in Heron \& Smyth, 2010), the aim is to recruit a total of 134 participants.

\section{Recruitment}

Recruitment started in May 2015 and takes place via advertisement (flyers) in healthcare centres and health fairs, online media (e.g., www.proefbunny.nl and www.digi-prik.nl, currently www.onderzoekmachine.nl), social media, and print media outlets. Furthermore, participants from previous studies on eating behaviour who indicated that they were interested in future study participation will be approached for participation. Interested candidates respond to advertisements via e-mail or through the study's website (www.thinkslim.nl).

\section{Randomization and procedure}

The study can be divided into five phases: 1) the phase from recruitment to randomization; 2) EMA-only Week 1, in which EMA data on eating behaviour and cognitions will be obtained; 3 ) the Intervention Phase; in which participants will actively follow a diet, either accompanied by the Think Slim intervention (immediate intervention group) or not (dietonly control group); 4) EMA-only Week 2: a second week in which EMA data on eating behaviour and cognitions will be obtained; and 5) the Follow-Up Phase: the period covered by assessment at 3 and 12 months post-intervention, and by the reception of the Think Slim intervention by the diet-only control group. In each phase, different modules of the Think Slim app will be active. The flow of the trial, measures and intervention components are graphically depicted in Figure 1. Each phase will be described in detail below. During the trial, participants are allowed to contact the research staff via e-mail and telephone to discuss any technical or personal issues that might be of influence on study participation. In addition, telephone contact is scheduled prior to the onset of each new phase. Care will be taken to avoid biasing participants during this communication, by referring to the online training and app manual whenever content-related questions arise. 


\section{Phase 1: from recruitment to randomization}

After initial response to the advertisements, interested candidates will be requested to complete an online screening questionnaire assessing in- and exclusion criteria. Eligible participants will receive further study information via e-mail and telephone. This will allow candidates to ask questions and to consider their study participation well before they agree to participate. Participants who are not eligible for participation will be informed via e-mail about the reason(s) for exclusion and will be provided with an opportunity to correspond with the research staff.

After agreeing to participate, participants will provide digital informed consent. Subsequently, participants will be contacted to plan a one-day technical training via the app shortly afterwards, and will be sent a manual for the app via postal mail and via e-mail. The purpose of the technical training is to ensure that the app is working and to minimize the influence of differences in technical aptitude.

\section{App functionality during the technical training}

During the technical training, the EMA module and Graphical Overview Feedback module of the app will be active. The EMA module of the Think Slim app will be used to obtain real-time data of eating-related variables. Items that are part of the EMA questionnaires include measures of food desire strength, specific food desires, emotions, cognitions, locations, activities, social company and food consumption. During the training, EMA will be performed at two separate instances: (1) eating events (event sampling), and (2) eight times pseudorandomly throughout the day (time-contingent sampling). Sampling of an eating event will be initiated by the participant him/herself, whereas time-contingent sampling points will be generated by the app. Note that cognitions and food consumption will only be assessed during eating event sampling. Otherwise the eating event samples and time-contingent samples are the same. The first time-contingent sample of the day will also assess sleep duration and quality. Beverage consumption will be assessed in a separate assessment, which will occur prior to bedtime.

In addition to the EMA module, graphical overview feedback will be accessible in a separate section of the app. The feedback will represent pie charts of the activities and locations assessed at eating event samples by the participant, separately for foods that are considered healthy and unhealthy. In addition, a line graph of desire strength and strength of various emotions, presented relative to time, will be available. The graphical overviews will continuously be updated, as soon as new EMA data is entered into the app. To generate the overviews, at least one eating event sample is necessary.

\section{Randomization}

After completing the technical training, all participants will be contacted by the researchers to discuss possible issues that occurred during the training. Subsequently, randomization 
will take place. Participants will be randomly assigned to either the immediate intervention group or the diet-only control group using computer generated randomization via Research Randomizer (www.randomizer.org). More specifically, randomization is pre-stratified by age group (18-30; 31 - 40; 41 - 50; 51 - 60) and gender. For each age group, separately for male and female participants, each participant will be assigned a randomly generated number. Half of the participants with the largest randomly generated numbers will be assigned to the immediate intervention group, whereas the other half will be assigned to the dietonly control group. Note that participants who indicate to be unavailable during a specific time period, due to for example holidays, will be manually allocated to the condition that matches with their availability. Blinding participants and researchers is not possible in the current setup.

\section{Phase 2: EMA-only week 1 (week 1)}

The trial starts with an EMA-only week. At the start of this week, all participants (immediate intervention group and diet-only control group) will fill out a baseline assessment consisting of several self-report questionnaires (specified below, under 'measures') about eating styles, eating disorder pathology, general psychological distress, self-esteem and dysfunctional thinking. In addition, participants will obtain an independent measure of their BMI. No further intervention will take place.

\section{App functionality during the first EMA-only week}

During the entire week, the EMA module and Graphical Overview Feedback module of the app will be active, identical to the technical training.

\section{Phase 3: Intervention Phase (week 2- 7)}

After the first EMA-only week, the Intervention Phase starts. This phase has a total duration of six weeks (week $2-7$ ).

\section{$\underline{\text { Diet }}$}

During the Intervention Phase, all participants (immediate intervention group and diet-only control group) are asked to actively follow a calorie-restrictive diet of their own choice. Supervision of the diet by a dietician or health-care professional is not allowed. Instead, all participants select a diet coach (friend or family member). Participants will also develop an exercise plan. General information about dieting and exercising is provided as part of two online sessions in the first week of the Intervention Phase. At the end of the first week, participants are asked to finalize their choice. Aside of the two online sessions, no further information about selecting a diet is provided. 


\section{Cognitive Intervention (for immediate intervention group only)}

The cognitive intervention will be aimed at changing obesity-related dysfunctional thinking and at increasing self-esteem. It consists of ten individual online sessions on a computer (CCBT), and a module in the app. The computer sessions are included because CBT requires explanation of the theoretical model and therapeutic concepts that are too comprehensive to provide via an iPhone app, due to screen size limitations that prevent a convincing digital presentation of the intervention. Therefore, the online sessions provide participants with the necessary information on the cognitive model and cognitive techniques, whereas the app is suitable to practice these skills in daily life.

Table 1. Summary of the contents of the CCBT sessions.

\begin{tabular}{|c|c|c|}
\hline$\#$ & Theme & Description \\
\hline 1 & Introduction & $\begin{array}{l}\text { - Information on weight-gain and weight-loss } \\
\text { - Introduction of the cognitive model and the concept of } \\
\text { dysfunctional thinking } \\
\text { - Information on how to select a diet, diet coach and exercise plan }{ }^{\text {a }}\end{array}$ \\
\hline 2 & Motivation & $\begin{array}{l}\text { - Personal reasons for losing weight } \\
\text { - Realistic weight loss goals* } \\
\text { - Identifying and responding to sabotaging cognitions about dieting } \\
\text { - Personal list of sabotaging cognitions about dieting }\end{array}$ \\
\hline 3 & Coping with food desire & $\begin{array}{l}\text { - Information on the difference between hunger and food desire } \\
\text { - Tips on how to deal with food desire } \\
\text { - Personal list of distraction techniques }\end{array}$ \\
\hline 4 & Identifying dysfunctional cognitions & $\begin{array}{l}\text { - Identifying dysfunctional cognitions about eating } \\
\text { - Introducing the concept of dietary temptations } \\
\text { - Instruction on how to do this in the app }\end{array}$ \\
\hline 5 & $\begin{array}{l}\text { Responding to dysfunctional } \\
\text { cognitions }\end{array}$ & $\begin{array}{l}\text { - Introduction of socratic questioning } \\
\text { - Information on how to construct functional alternative cognitions } \\
\text { - Instruction on how to do this in the app. } \\
\text { - Choosing a diet and exercise plan } \text { a }^{\text {*** }}\end{array}$ \\
\hline 6 & Thinking Errors & $\begin{array}{l}\text { - Summary of basic CBT skills } \\
\text { - Introduction of concept of thinking errors } \\
\text { - Instruction on how to do this in the app }\end{array}$ \\
\hline 7 & Interim evaluation & $\begin{array}{l}\text { - Evaluation on progress so far } \\
\text { - Increasing motivation } \\
\text { - Learning to compliment oneself }\end{array}$ \\
\hline 8 & Self-Esteem: Body & - Positive body exposure \\
\hline 9 & Self-Esteem: Person & - Designing a positive portrait of the self \\
\hline 10 & Evaluation and Relapse Prevention & $\begin{array}{l}\text { - Summary of intervention } \\
\text { - Evaluation of intervention } \\
\text { - Creating a relapse prevention plan }\end{array}$ \\
\hline \multicolumn{3}{|c|}{$\begin{array}{l}\text { Note: the content of these sessions is derived from several CBT protocols (Beck, 2007; Werrij et al., 2009; Jansen \& Mulkens, 2015). }{ }^{\text {a }}= \\
\text { component is completed by immediate intervention and diet-only control groups simultaneously; }{ }^{*}=\text { information is transferred to the } \\
\text { app for review; }{ }^{* *}=\text { information will be sent to participants by e-mail; In each session, the level of dysfunctional thinking will be assessed } \\
\text { by believability ratings (VAS-scales) of nine frequently occurring dysfunctional cognitions about eating. }\end{array}$} \\
\hline
\end{tabular}


There are a total of ten online sessions of approximately 25 minutes that will be delivered via an in-house web-environment for online therapy and questionnaire research (SOTO). To start each session, participants receive an e-mail with a link to the SOTO web-environment. The sessions are based on several CBT for weight loss protocols (Beck, 2007; Werrij et al., 2009; Jansen \& Mulkens, 2015). They are designed to be provided in addition to a standard calorie-restrictive diet. Table 1 contains a brief summary of each session's contents. In the first week of the Intervention Phase, five sessions will take place. In these sessions, participants will determine reasons for losing weight, set dietary goals, choose a diet, make an exercise plan, and make a list of techniques to distract from food craving. All information except the choice of diet and exercise plan will be accessible for review in the app. Furthermore, participants will be familiarized with the cognitive model and basic cognitive techniques. Participants will learn how to identify and evaluate dysfunctional cognitions, and how to replace these with alternative cognitions that are more helpful towards eating behavior and dieting. For the first week, all five sessions will need to be completed at the end of the week. Participants who do not complete all five sessions will be considered dropouts. Participants will be sent reminder e-mails in case they lag behind and will be contacted by the research staff in case they are close to being considered dropouts.

From the second week until the last (week 7) of the Intervention Phase, participants will complete additional cCBT sessions at the end of each week (sessions 6 - 10). In these sessions, cognitive skills are reviewed, and participants are encouraged to stay motivated. Furthermore, self-esteem is addressed in two sessions, focusing on the body and on personality characteristics. In the final session special attention is paid to evaluation and relapse prevention. All online CCBT sessions will be completed without therapist involvement. Participants are allowed to lag behind one session, and will be considered dropouts if more than one session is not completed on time. At the beginning of each session, dysfunctional thinking will be assessed via a self-report questionnaire. Participants in the diet-only control group will not receive any cognitive intervention during the Intervention Phase.

\section{App functionality during the Intervention Phase}

Participants in the diet-only control group will not be able to use the app during the Intervention Phase. For these participants, all app functionality will be blocked. Instead, the app will display the number of days remaining until the end of the waiting period. Eight days before the end of the waiting period, the app will send a notification informing participants that the end of the waiting period is near.

For participants in the immediate intervention group, app functionality in the first week of the Intervention Phase will consist of the EMA module and the Graphical Overview Feedback module, similar to the training and the first EMA-only week. In addition, the Adaptive Feedback module will be active. The Adaptive Feedback module provides participants with feedback if they are at risk for overeating. The feedback message consists of a generic warn- 
ing and a behavioural advice. Detection of a participant's risky moments is based on the answers provided by the participant on the EMA items. To estimate when the participant is likely to eat something unhealthy in the time-period directly following a completed timecontingent sample, the sample data is compared to a set of allocated pre-existing rules that indicate what combinations of states of variables (e.g., scoring high on desire + being at home + feeling bored + feeling calm) are predictive of unhealthy eating. If there is a match between the time-contingent sample and one of the rules, the participant receives adaptive feedback messages via the Think Slim app.

To provide adaptive feedback, the Think Slim app uses 65 rules that were obtained via a computational decision-tree algorithm (adapted for EMA data) used on a dataset from a previous EMA study that had an almost identical EMA protocol (Boh et al., 2016). Each of the 65 rules represents states of eating-related variables that were most frequently reported together right before a healthy or unhealthy eating event, as reported by participants of that study. More specifically, participants were divided to six groups according to their rule triggering occurrence and each group is represented by a set of rule that describes $80 \%$ of the eating behaviour of participants in the group (thus removing rules with low occurrence and keeping only those with high predictive value). For the current study, each participant will be allocated one of the six rule triggering groups based on EMA data from the first EMAonly week. At the end of the third week of the Think Slim intervention, EMA sample data from the first three weeks of the Intervention Phase will be used to update the allocation of participants to the rule triggering groups.

From the second week of the Intervention Phase until the end of the intervention, the app functionality will be further expanded. First, participants will be instructed to also assess moments of dietary temptation (event sampling) in the app. Participants will be instructed

Table 2. Cognition types included in the Think Slim app and the categories they belong to.

$\begin{array}{lll}\text { Category } & \text { Cognition type } & \text { Example } \\ \text { Neutral } & \text { Description of an eating event } & \text { "Time for breakfast!" } \\ & \text { Hunger } & \text { "I am very hungry, time to eat." } \\ & \text { Desire and taste } & \text { "I really want this chocolate bar." } \\ & \text { Energy needed } & \text { "I won't have time to eat later." } \\ \text { Functional } & \text { Healthy intention } & \text { "Apples are good for me." } \\ & \text { Successful control } & \text { "Everyone's eating cake, but l'm going for a pear." } \\ \text { Dysfunctional } & \text { Negative emotions } & \text { "I feel awful, maybe this helps." } \\ & \text { Positive emotions } & \text { "I'm trying to relax with snacks." } \\ & \text { Social activities and pressure } & \text { "Everyone's eating cake. I will join them." } \\ & \text { Reward } & \text { "I deserve this after all the hard work!" } \\ & \text { Control failure } & \text { "I just can't resist." }\end{array}$

Note: These cognition types are based on previous research (Boh et al., 2016). 
in an online CCBT session about what qualifies as a dietary temptation. The EMA items assessed during moments of dietary temptations are similar to those assessed during an eating event, except for the food consumption item: food desire strength, specific food desires, emotions, location, company, and activities. Second, the Cognition Evaluation module will be active. The purpose of this module is to identify and evaluate dysfunctional cognitions in daily life. Table 2 contains an overview of the cognitions included in the app and whether or not these are considered dysfunctional. The module designed to work in conjunction with the online CCBT sessions: the module requires knowledge of the basic cognitive skills that are provided in online sessions $1-5$.

When a dysfunctional cognition is selected by a participant in the Cognition Evaluation module of the app during an eating event or dietary temptation, the participant is encouraged to critically evaluate the selected cognition via socratic questions, which aim to guide the evaluation process. For the ease of evaluation, the cognition is presented in a conditional (if - then) format. After the evaluation, the participant is asked to aggregate the information obtained in the evaluation section and use this information to formulate a helpful and realistic response to the initial dysfunctional cognition. Subsequently, the believability of the new cognition is rated. To conclude, the participant is asked to take another look at the initial cognition and rate believability again. The idea is that after evaluation, the believability - and therewith the influence - of the initial cognition decreases. All evaluated cognitions remain accessible in the app for future reference. If a functional or neutral cognition is selected in the app by a participant, the app also provides feedback. In case of an eating event, this feedback will be a generic message (e.g., "Enjoy your meal"). In case of a dietary temptation, an appropriate cCBT-based feedback message will be provided to the participant. This feedback message will refer back to what was learned during the CCBT sessions if the selected cognition is considered dysfunctional.

Lastly, at the last day of week 7, a post-intervention assessment will take place during which participants will fill in self-report questionnaires identical to the baseline assessment. Participants are also instructed to obtain a second measurement of weight during week 8 , under identical circumstances as during the first EMA-only week.

\section{Phase 4: EMA-only week 2 (week 8)}

After the intervention phase, all participants (immediate intervention group and diet-only control group) enter a second EMA-only week. This week is identical to the first EMA-only week for all participants.

\section{Phase 5: Follow-Up Phase}

At the end of the second EMA-only week (week 8), participants in the immediate intervention group will be debriefed about the purpose of the trial. Furthermore, they will receive a monetary reward of $€ 50$,- in coupons. After that, their Follow-Up (FU) Phase starts. The FU 
Phase has a total duration of 12 months. Participants are invited to fill out questionnaires (identical to the baseline and post-intervention assessments) and to obtain independent BMI measurements at two time-points during this period: at 3 and 12 months post-intervention. They will receive an additional €20,- in coupons per completed FU measurement.

For the diet-only control group, the procedure after the second EMA-only week (week 8) is different. Participants allocated to this group will now receive the Think Slim intervention (week 9 - 14), followed by a third EMA-only week (week 15) and a debriefing. The content and procedure of the Think Slim intervention received by the diet-only control group is identical to that received by the immediate intervention group, except that information concerning the diet and exercise plan is not included. Furthermore, the third EMA-only week is identical to the other two EMA-only weeks. After that, the 12-month FU Phase starts for this group. The procedure of the FU Phase for the diet-only control group is identical to the procedure of the immediate intervention.

\section{MEASURES}

Several instruments will be used to assess the effects of the Think Slim intervention on BMI and secondary outcomes. Data are obtained by means of self-report instruments, measures taken by health-care professionals, and EMA data. Figure 1 depicts when each measure is obtained during the flow of the study.

\section{Body Mass Index}

Participants are instructed to obtain multiple measurements of body weight and one measurement of height. These measurements are used to calculate BMI. To ensure objectivity, participants are asked to obtain the measurements via a healthcare professional (e.g., a general practitioner, or a physical therapist), or at the university (Think Slim research staff). Participants are further requested to obtain all measurements under identical circumstances (i.e., the same general practitioner or physical therapist).

\section{Patterns of eating behaviour}

To gain insight in (changes in) eating patterns, EMA data obtained during the first EMA-only week and second EMA-only week will be investigated.

\section{Dysfunctional thinking}

Dysfunctional thinking will be measured by believability ratings of nine frequently occurring dysfunctional cognitions about eating (e.g., "If everyone is eating a snack, then I should participate"). Participants are instructed to read each statement carefully, and to answer the question "How much do you believe in this cognition?" by scoring believability on a VAS 
ranging from 0 - 100; anchors "not at all" and "very much so". Cognitions are selected based on previous research (Boh et al., 2016) and observations in clinical practice.

\section{Eating styles}

The Dutch Eating Behaviour Questionnaire (DEBQ; van Strien, Frijters, Bergers, \& Defares, 1986) will be used to measure eating styles and consists of 33 items across three subscales: external eating, emotional eating and restraint. Each item of the DEBQ is rated on a 5-point Likert scale ranging from 1 (seldom) to 5 (very often). Item scores for the three subscales will first be summed separately to obtain an overall score per subscale. Then, for each subscale, the overall score will be divided by the number of subscale items to obtain the final average score per subscale. A high score on one of the subscales indicates a higher tendency to display the respective eating style. The DEBQ was previously found to be internally consistent overall, $a=0.79$ [61]. The subscales emotional eating ( $a=.89$ ), external eating ( $a=.078$ ) and restrained eating ( $a=.92$ ) were also found to be internally consistent (Evers, Marijn Stok, \& de Ridder, 2010).

\section{Eating disorder pathology}

The Dutch version of the Revised Eating Disorder Examination Questionnaire (EDE-Q; Fairburn \& Beglin, 1994; Dutch translation) will be used to measure eating disorder pathology. This self-report questionnaire consists of 22 items assessing eating pathology, and six items assessing diagnostic behaviours such as use of laxatives and purging. The items are scored across four subscales: restraint, weight concerns, shape concerns, and eating concerns. The eating pathology items of the EDE-Q are each scored on a 7-point Likert scale. The diagnostic behaviour items are scored by participants indicating the number of times in the past 28 days that the behaviour occurred. The test-retest reliability of the English version of the EDE-Q was found to be between $r=0.66$ and $r=0.94$ for each of the subscales, whereas the internal consistency of the EDE-Q subscales was found to be good, ranging from $a=0.70$ to $a=0.93$ (Berg, Peterson, Frazier, \& Crow, 2012).

\section{General psychological symptomatology}

The Brief Symptom Inventory (BSI: Derogatis \& Melisaratos, 1983; Dutch translation by De Beurs, 2006) will be used to assess general psychological symptomatology, and consists of 53 items. The BSI has nine subscales assessing different forms of psychological distress. Items of the BSI are scored on Likert scales ranging from 0 (not at all) to 4 (very much so). Scores for each subscale are calculated by averaging scores on the individual items belonging to that subscale. The total score for the BSI is calculated by summing all individual item scores. The BSI subscales were found to have satisfactory internal consistency, ranging from $a=0.71$ to $a=0.88$ (Andreu et al., 2008). In addition, the test-retest reliability is high, with the lowest score reported at $r=0.68$ (Andreu et al., 2008). 


\section{Self-Esteem}

The Self-Liking and Self-Competence Scale Revised (SLSC-R; Tafarodi \& Swann, 1995; Dutch translation by Vandromme, Hermans, Spruyt, \& Eelen, 2007), will be used to assess self-liking and self-competence as dimensions of self-esteem, and consists of 16 items, 8 for each dimension. Items are rated on 5-point Likert scales ranging from 1 (strongly disagree) to 5 (strongly agree). Overall scores per dimension are obtained by summing scores of individual items belonging to each dimension, and can range between 8 and 40 . Higher scores indicate more self-competence and self-liking than lower scores. Internal consistency of the subscales of the Dutch translation of the SLSC-R was found to be good, ranging from $a=.78$ to $a=.98$ (Vandromme et al., 2007). Split-half reliability for the subscales was also found to be good, ranging from $r=.75$ to $r=.92$.

\section{Acceptability and compliance}

Acceptability of the Think Slim intervention will be measured using a questionnaire that was developed for the current study. This questionnaire includes items on user-friendliness and perceived usefulness of the Think Slim intervention. Compliance with the EMA module in the app will be measured daily during the study by calculating how many time-contingent samples were completed by participants relative to the total number of time-contingent samples that were sent by the app, and by monitoring how often the Cognition Evaluation module of the app was used.

\section{DATA ANALYSIS}

Participant's flow from screening to randomization will be mapped out. Furthermore, pretreatment characteristics of the sample will be explored, and baseline differences between conditions will be examined in terms of size and clinical importance. Distributions of outcome variables will be inspected via histograms and boxplots. After that, intervention- and study-compliance will be determined, followed by an evaluation of participants'satisfaction with the Think Slim intervention.

To test the effectiveness of the Think Slim intervention, 2 (group: immediate intervention vs. diet-only control group) $\times 2$ (time: pre vs. post) ANOVAS will be run for the set of dependent variables (i.e., BMl, dysfunctional thinking, scores for questionnaires on eating styles, eating disorder pathology, general psychological symptomatology, and self-esteem). Long-term effects of the Think Slim intervention on the set of dependent variables will be examined using one-way repeated-measures ANOVAs collapsing over group (i.e., for all 102 participants), with four levels: pre-Think Slim, post-Think Slim, 3 month follow-up and 12 month follow-up. All analyses will be performed according to the intention-to-treat principle (Newell, 1992) and the completers principle. Lastly, time-lagged multilevel network analyses 
will be conducted on data obtained during the pre- and post-Intervention Phase EMA-only weeks, to assess changes in patterns of data that result in unhealthy eating (Borsboom \& Cramer, 2013; Spanakis et al., 2015). Changes in network connectivity between the pre- and post-Intervention Phase networks will be compared between both conditions. Of particular interest are changes in network connectivity and density related to unhealthy eating, in terms of graph analysis measures such as indegree, outdegree, betweenness, and centrality of network nodes.

\section{DISCUSSION}

Whereas short-term weight loss is often achieved in existing interventions for obesity, this weight loss is almost invariably followed by weight regain. Ecological momentary intervention may help reduce weight regain because it allows indefinite treatment and can assist obese people online (i.e., at moments of weakness). This article presented the design of an RCT comparing the effects of the Think Slim ecological momentary intervention with a diet-only control condition, in terms of (1) weight loss, (2) changes in self-report measures of dysfunctional thinking, eating styles, eating disorder pathology, general psychological symptomatology and self-esteem, and (3) changes in eating patterns. The trial includes follow-up measurements of weight at 3 months and at 12 months. The current study is, to the authors' knowledge, the first to test a completely online and app-based protocol of cCBT for obesity, with no therapist involvement. With the current study, an effort is undertaken to improve understanding of long-term weight loss and the influence of cognitions and behaviour on achieving such long-term weight loss. With this we aim to contribute to the improvement of interventions for eating behavior and obesity.

\section{Trial status}

Recruiting.

\section{Abbreviations}

CBT: cognitive behavioural therapy; CCBT: computerized cognitive behavioural therapy; BMI: body mass index; RCT: randomised controlled trial; EMA: ecological momentary assessment; EMI: ecological momentary intervention; ANOVA: analysis of variance; DEBQ: Dutch eating behaviour questionnaire; EDE-Q: eating disorder examination questionnaire; BSI: brief symptom inventory; SLSC-R: self-liking and self-competence scale revised

\section{Competing interests}

The authors declare that they have no competing interests. 


\section{Acknowledgments and funding}

The authors thank Jessie Beerthuyzen, Lianne van Cleef and Dorien Blezer for help during the preparations of the study. The authors thank Michiel Vestjens and Charlie Bonnemayer for adapting the SOTO web-environment to the Think Slim intervention. This study is funded by grant 12028 from Stichting Technische Wetenschappen (STW), Nationaal Initiatief Hersenen en Cognitie (NIHC), Nederlandse Organisatie voor Wetenschappelijk Onderzoek (NWO) and Philips under the Partnership programme Healthy Lifestyle Solutions, awarded to the last author (AR).

\section{Authors' contributions}

Funding for the study was obtained by AR, GW, CN and AJ. All authors contributed to the design of the study. VK is responsible for programming the Think Slim app. BB and AR are responsible for the design of the EMA protocol and graphical overview feedback app module, in close collaboration with VK and AJ. GS is responsible for the adaptive feedback app module, in close collaboration with $B B, G W, A R, V K, C N$, and LL. LL is responsible for the online CCBT sessions, in collaboration with $B B, A R$, and $A J$. $L L$ and $B B$ are responsible for the cognition evaluation app module, in close collaboration with AR and AJ. Recruitment and data-collection will be done by BB. Data-analysis will be carried out by BB and GS, in collaboration with $A R, G W, L L$, and AJ. BB drafted the current manuscript in close collaboration with $A R$ and $L L$, and all authors approved the final version. 



\section{Chapter 5}

Randomized Controlled Trial of a

Cognitive Behavioural Therapy-

based Ecological Momentary

Intervention for Obesity

B. Boh

A. Jansen

L. H. J. M. Lemmens

C. Nederkoorn

G. Spanakis

A. Roefs

(in preparation). Randomised Controlled Trial of a

cognitive behavioural therapy-based ecological momentary intervention for obesity. 


\section{ABSTRACT}

Objective. The present study investigated the acute effectiveness of a 6-week individually tailored autonomous CBT-based ecological momentary intervention (Think Slim). It was hypothesized that (1) Think Slim would result in greater BMI reduction and eating-related cognitive and emotional improvement than a self-guided diet-only control, and (2) that participants with higher treatment adherence would perform better on the outcome measures.

Method. Overweight adult participants were randomized to Think Slim ( $n=46$ analysed) or the control condition ( $n=50$ analysed). Think Slim consisted of an iPhone app and ten web-based sessions. Outcome measures were BMI, self-reported dysfunctional cognitions and emotions / states, eating styles, eating disorder psychopathology, psychiatric symptoms, and self-esteem.

Results. Participants in both conditions had significant but not significantly different BMI reductions, increases in self-liking, and reductions in eating disorder psychopathology. Participants in Think Slim had a greater reduction in dysfunctional cognition belief and frequency, emotional eating, and external eating, than those in the control condition. Higher dietary adherence was associated with greater BMI reduction.

Conclusion. BMI reduction was statistically significant, but modest, and comparable to other CBT for obesity trials. The cognitive and emotional improvements reached by Think Slim may aid in long-term weight-loss maintenance. 
Keywords: Cognitive behavioural therapy, randomized controlled trial, ecological momentary assessment, ecological momentary intervention, weight loss, obesity

\section{INTRODUCTION}

Obesity is a pandemic that is associated with adverse health effects, such as cardiovascular disease, type-2 diabetes, and osteoarthritis (Dixon, 2010), adverse psychological effects, such as stigma (Puhl \& Heuer, 2010), and large annual medical care costs (Cawley \& Meyerhoefer, 2012). Obesity treatment may benefit from tailoring to individual experiences (Blundell et al., 2005; Brownell, 1991) and from interventions that focus on eating-related thoughts and emotions (Carter \& Jansen, 2012; Jansen, Houben \& Roefs, 2015). The present randomized controlled trial $(\mathrm{RCT})$ investigated the effectiveness of an individually tailored ecological momentary intervention (EMI), based on principles of cognitive behavioural therapy (CBT), on Body Mass Index (BMI), eating-related dysfunctional cognitions, eating styles, eating disorder psychopathology, self-esteem, and general psychiatric symptoms, in overweight and obese $\left(\mathrm{BMI}>25 \mathrm{~kg} / \mathrm{m}^{2}\right)$ participants.

There is currently no standardized effective treatment for obesity (Brownell, 2010; Carter \& Jansen, 2012). Weight loss is almost always followed by weight regain (Curioni \& Lourenço, 2005; Franz et al., 2007). To investigate how to increase the effectiveness of obesity treatment, it may prove beneficial to adopt principles of CBT for the treatment of obesity (Carter \& Jansen, 2012; Jansen et al., 2015; Karhunen et al., 2012). The CBT model of obesity postulates that dysfunctional cognitions and emotions related to eating, weight, and shape concerns, underlie a failure to control or inhibit food intake (Gade, Hjelmesæth, Rosenvinge, \& Friborg, 2014; Jansen et al., 2015; Nauta, Hospers, Jansen, \& Kok, 2000), and thus promote overeating. Overeating provides short-term beneficial effects, such as enjoyment. However, it also reinforces the belief in dysfunctional cognitions, and negatively affects belief in selfefficacy, which in turn makes subsequent overeating more likely. To break this vicious cycle, dysfunctional cognitions should be replaced by functional alternatives (e.g., "If I refuse this offer of food, I will feel happier about it in the long-term").

CBT for obesity aims to train clients to identify dysfunctional cognitions that precede overeating behaviour, to evaluate them, and replace them with functional alternative cognitions (Beck, 2007). CBT is a relatively effective and standardized treatment for eating disorders (Fairburn et al., 2009; Fairburn, Cooper, \& Shafran, 2003), binge eating (Ashton, Drerup, Windover, \& Heinberg, 2009), and dysfunctional eating behaviour in bariatric surgery patients (Gade et al., 2014). So far, most trials found that CBT for obesity resulted in moderate but statistically significant weight loss, which, contrary to control conditions, was maintained at follow-up measurements (Stahre \& Hällström, 2005; Stahre, Tärnell, Håkanson, \& Hällström, 2007; Werrij et al., 2009). However, in one trial, participants in the CBT condition did regain 
the lost weight at follow-up (Cooper et al., 2010). So, more trials are needed to explore how weight loss and weight loss maintenance success can be increased via CBT.

One potential improvement, is to help dieters better deal with food desire and temptations to eat throughout the day (Carels et al., 2001; Carels, Douglass, Cacciapaglia, \& O'Brien, 2004; McKee, Ntoumanis, \& Taylor, 2014). This can be done by intervening promptly when food temptations occur, or when it is likely that overeating will take place. Additionally, as obesity is sometimes considered a chronic condition (Bray, 2004; Perri, 1998; Rippe et al., 1998), indefinite treatment may increase long-term weight maintenance success. One way to accomplish these treatment innovations is to use the internet and mobile technology.

Obesity treatment is increasingly being provided via the internet (Andersson, 2009; Bennett \& Glasgow, 2009). Effective internet interventions include elements of CBT, selfmonitoring of (eating) behaviour and progress tracking, options for social support, and individualized feedback (Krukowski, Harvey-Berino, Ashikaga, Thomas, \& Micco, 2008; Saperstein, Atkinson, \& Gold, 2007; Sherrington et al., 2016). Mobile devices such as smartphones and personal digital assistants have also been used to provide obesity treatment in clients' daily lives, via ecological momentary intervention (EMl; Heron \& Smyth, 2010). To be able to intervene at critical moments, EMI relies on knowledge of the factors that are typically related to a client's disordered behaviour. Such knowledge is often, but not necessarily, attained through ecological momentary assessment (EMA; Shiffman, Stone, \& Hufford, 2008) whereby clients are periodically prompted with disorder or treatment-related questions. Existing EMls for obesity have provided mixed results, with some studies finding significant weight loss (Agras, Taylor, Feldman, Losch, \& Burnett, 1990; Burnett et al., 1985; Patrick et al., 2009), which was maintained at follow-up in one study (Burnett et al., 1985) but not in another (Agras et al., 1990). One EMI did not result in weight loss at all (Shapiro et al., 2012). Also, EMIs with no therapist support have been used to effectively promote healthy food intake (Atienza, King, Oliveira, Ahn, \& Gardner, 2008; Nour, Chen, \& Allman-Farinelli, 2015), and prevent weight gain in young adults (Partridge et al., 2015). So, EMI may have the potential to help individuals adjust their eating behaviour and lose weight by providing treatment promptly in daily life.

Although not explicitly investigated for weight loss, CBT is especially suitable for EMI, as it consists of a protocolized treatment program (Beck, 2011), which has been translated to self-help manuals (e.g., Beck, 2007), and is well-investigated. Studies investigating the use of C(B)T-based EMIs in the context of smoking cessation, anxiety, alcohol use, physical activity and eating disorders have resulted in significant health improvements (See Heron \& Smyth, 2010 for a review).

The present RCT investigates the effectiveness of an EMI, which is designed to intervene promptly when overweight $\left(\mathrm{BMI}>25 \mathrm{~kg} / \mathrm{m}^{2}\right)$ participants are at risk of overeating, and to teach participants how to deal with food temptations, dysfunctional cognitions, and a low self-esteem (the intervention is called Think Slim; see Boh et al. (2016) for the protocol). The 
Think Slim EMI is based on CBT for obesity (Beck, 2007; Werrij et al., 2009; Jansen, Mulkens \& Jansen, 2011; Jansen \& Mulkens, 2015), and consists of an iPhone app and web-based internet sessions.

The main aim of the present study is to investigate reduction in BMI. It is hypothesized that Think Slim participants will have a greater reduction in BMI than the self-guided diet-only control condition. For the secondary outcome measures, it is hypothesized that Think Slim participants will have a greater reduction in self-reported belief in dysfunctional cognitions about food and eating, and greater improvements on dysfunctional eating styles, eating disorder psychopathology, psychiatric symptomatology, and self-esteem, as assessed by questionnaire measures and EMA, than the control condition. In addition, as previous research found that dietary adherence is a better predictor for dieting success than the macronutrient content of a diet (Sacks et al., 2009), it is expected that weight loss and cognitive and emotional improvement, will correlate positively with treatment compliance. For the present study, the acute effects (i.e., baseline to post intervention) of the Think Slim intervention are investigated. The follow-up results will be published in a forthcoming paper.

\section{METHOD}

\section{Trial design and study overview}

The present study has been pre-registered at the Dutch Trial Register, part of the Dutch Cochrane Centre (NTR5473). Details about the design and protocol of the present study can be found in the study's protocol (Boh et al., 2016), and only a summary will be presented here. It was a randomised controlled superiority trial with a parallel group design, investigating the acute effectiveness of an EMI of CBT for weight loss (the Think Slim intervention; see Interventions) as compared to a self-guided diet-only control condition. The Think Slim condition, which consists of the Think Slim intervention and a self-guided diet, is compared to a self-guided diet-only control condition. Both interventions spanned six weeks. The trial was conducted online, via an iPhone application and treatment website. The Think Slim intervention did not include therapist support, but instead was designed to tailor to participants' individual circumstances automatically, via a decision tree algorithm (Spanakis et al., 2016). Ethical approval was obtained from the Ethics Committee of the Faculty of Psychology and Neuroscience of Maastricht University (The Netherlands). All participants provided informed consent for participation by signing a digital statement, and were aware that they could stop participating at any time.

\section{Participants and recruitment}

The flow of the trial can be found in Figure 1. Overweight and obese participants (BMI $>25 \mathrm{~kg} / \mathrm{m}^{2}$ ) between 18 and 60 years of age, with an iPhone, were recruited in the gen- 
eral population. Advertisements were placed in newspapers, on recruitment websites, and on social media. Exclusion criteria were: (1) presence of an eating disorder, (2) use of appetite-influencing medication, (3) enrolment in other obesity treatments, treatment for depression, and/or in other research on eating behaviour, (4) pregnancy, and (5) diabetes. To determine the presence of an eating disorder, participants were screened using the Eating Disorder Examination Questionnaire (EDE-Q; Fairburn \& Beglin, 1994), with a threshold of 4.27, which represents the 95th percentile of the obese population (Aardoom, Dingemans, Slof Op't Landt, \& Van Furth, 2012). The threshold was deliberately high, as CBT for obesity was previously found to result in reduction in eating disorder psychopathology (Werrij et al., 2009). Three participants scored above the threshold, and their participation was discussed

\section{Enrollment}

Assessed for eligibility $(n=300)$

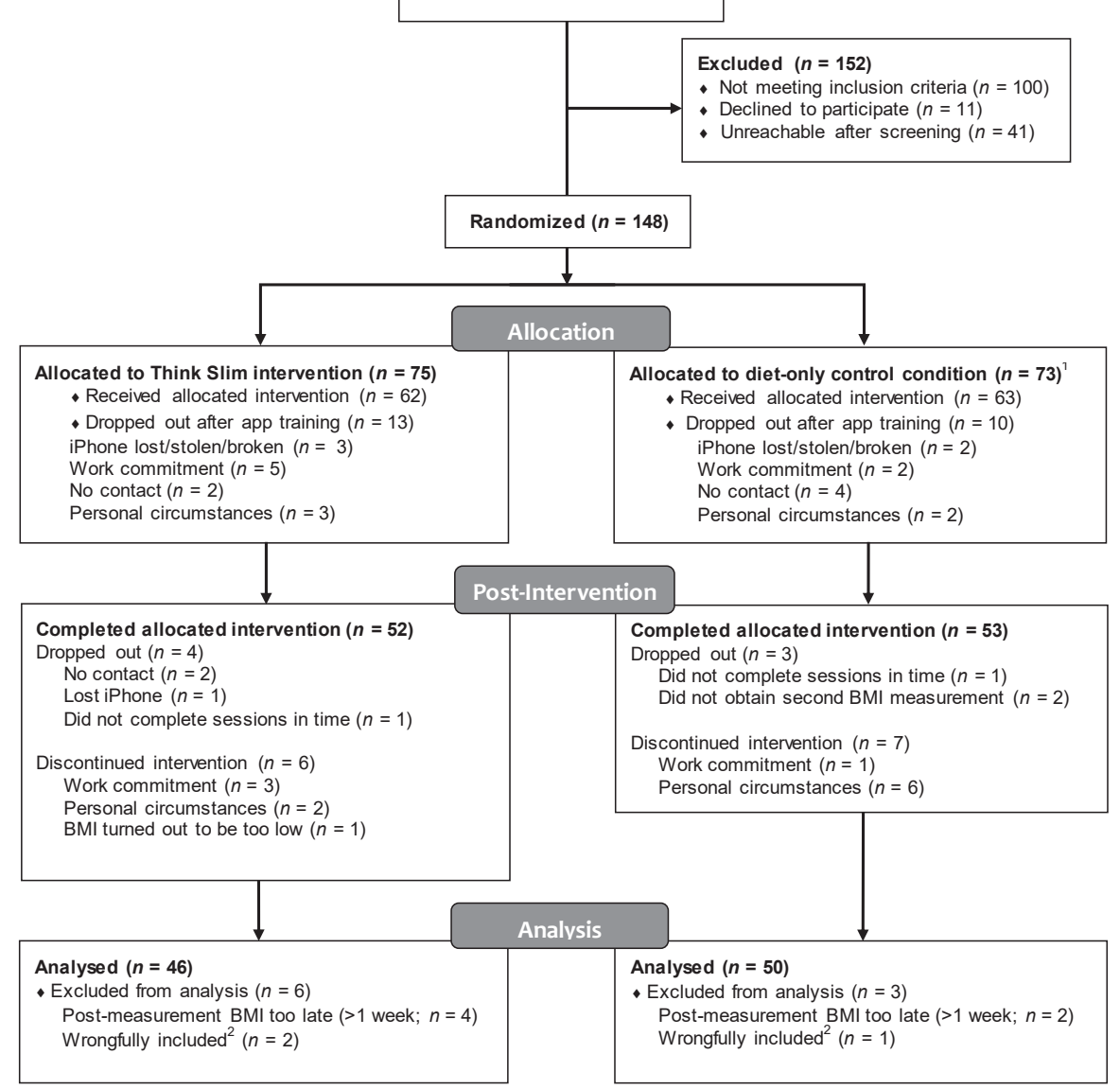

Figure 1. Participant flow from recruitment to baseline.

Note. 'Participants in the self-guided diet-only control condition were instructed to follow a diet and exercise plan of choice during the waiting period.

${ }^{2}$ These participants did not have a BMI $>25 \mathrm{~kg} / \mathrm{m}^{2}$ at the baseline supervised BMI measurement. 
by the research team (AJ, AR, BB and LL. AJ and $L L$ are also CBT therapists). After careful consideration, it was decided participating in the present study would not be harmful to these individuals, so there was no reason to exclude them from participation. An a-priori power analysis using G*Power (Faul, Erdfelder, Lang, \& Buchner, 2007) determined that 102 participants (51 per condition) would provide $80.6 \%$ power (one-tailed, $a=0.05$ ) to detect a medium effect size of Cohen's $d=0.5$ for differences between conditions (Boh et al., 2016). A total of 105 participants who were enrolled in the study completed their allocated intervention. However, before analyses, it was decided to exclude nine of them from further analyses, because (1) the second BMI measurement was received two or more weeks late $(n=6)^{2}$, or (2) they were diagnosed with an appetite-influencing medical condition (e.g. see White, 2010) during the intervention phase $(n=3)$. The final sample, therefore, consisted of 96 participants (Think Slim condition: $n=46$; self-guided diet-only control condition: $n=50$ ).

\section{Measures}

Psychometrics of the included questionnaire measures, including minimum and maximum scores, and number of items, can be found in the protocol of the present trial (Boh et al., 2016).

Primary outcome: BMI. Participants were instructed to obtain measurements of height and weight performed either by healthcare professionals or by researchers at the university, to compute BMI. The measurements were filled out on a form and signed by the healthcare professional, and then sent to the research staff by e-mail. If it was not possible to obtain an independent measurement, participants were, as an exception, allowed to provide selfreport measurements of height and weight. Eleven participants provided self-report(s) of BMI.

\section{Secondary outcomes}

All secondary outcomes were assessed at baseline and post-intervention, except belief in dysfunctional cognitions, which was also assessed at six time points during the intervention, for the Think Slim condition.

Dysfunctional cognitions. To assess the belief in eating-related dysfunctional cognitions, a questionnaire was developed, which includes nine frequently occurring dysfunctional cognitions about food and eating (Core Cognitions Questionnaire; CCQ); see Figure 2. Items were selected based on data of a previous study (Boh et al., 2016), and on the clinical exper-

2 Including these six participants in the analyses did not yield substantially different results for all outcome measures (BMI, self-report questionnaires, and EMA data). Note that, for the EMA data, three of these six participants could not be included due to not conducting EMA during the post-intervention week $(n=1)$ or not reporting any eating events during the post-intervention week $(n=2)$. 
tise of two of the authors (AJ/LL). Believability of each cognition was rated on a VAS ranging from 0 (not at all believable) to 100 (very believable). The overall questionnaire score was calculated as the average of all 9 items. In addition, all 9 items were analysed separately, to determine if there were any items that deviated from the others.

Eating Styles. To assess eating styles, the Dutch Eating Behaviour Questionnaire (DEBQ; van Strien, Frijters, Bergers, \& Defares, 1986) was used. The DEBQ assesses three eating styles: emotional eating (overeating in response to negative emotions), external eating (eating in response to environmental food-related cues), and restrained eating (attempting to control food intake). Items are rated on a 5-point Likert scale, ranging from 1 (seldom) to 5 (very often). Higher scores point to a self-reported more frequent occurrence of the measured type of eating behaviour.

Eating Disorder Psychopathology. The Eating Disorder Examination Questionnaire (EDE-Q; Fairburn \& Beglin, 1994) was used to assess eating disorder psychopathology. Items of the EDE-Q are scored on a 7-point Likert scale assessing frequency and severity over the past 28 days, ranging from 1 (not at all) to 7 (every day), and subscales represent restrained eating, eating concerns, weight concerns, and shape concerns. In addition, the EDE-Q contains six items to assess binge eating and intake compensation behaviour. An overall high score on the EDE-Q indicates more eating disorder psychopathology.

Psychiatric symptoms. The overall score on the Brief Symptom Inventory (BSI; Derogatis \& Melisaratos, 1983) was used to assess the occurrence of diverse psychiatric symptoms. Items of the BSI are scored on Likert scales ranging from 0 (not at all) to 4 (very much so). Higher scores indicate increased presence of psychiatric symptoms.

Self-liking and Self-Competence. The Self-Liking and Self-Competence (SLSC) scale assessed self-liking and self-competence as dimensions of self-esteem (Tafarodi \& Swann, 1995). Items of the SLSC are scored on Likert scales ranging from 1 (strongly disagree) to 5 (strongly agree). High scores indicate a high self-liking and/or self-competence assessment.

Ecological Momentary Assessment (EMA). The EMA procedure is described in more detail in the study protocol (Boh et al., 2016). It included both signal-contingent and event-related sampling. For the signal-contingent sampling, every waking day was divided into an average of eight 2-hour time windows during which the app prompted participants with EMA questions on food desire, emotions, states (bored / tired), locations, social company and activities. Participants were instructed to complete each sample within a timeframe of 20 minutes after receiving the prompt. For the event sampling, participants were instructed to self-initiate and complete an assessment prior to any eating event. During a specific period of the intervention (see Interventions for more details), participants of the Think Slim condition also assessed dietary temptations. At the event-samples (i.e., eating events and temptation events), participants were asked to (1) complete the signal-contingent prompt EMA questions, but also (2) select their current cognition related to eating (e.g., "When I do not stick to my diet, I may as well continue eating") from a list of 35 common eating-related 
cognitions (adapted from clinical expertise, and Boh et al., 2016), (3) score belief in the selected cognition, and (4) report their food intake via an overview of 20 icons containing common food types in the Western diet. All completed eating event and dietary temptation assessments were stored in a logbook in the app, and immediately accessible for review.

Compliance. Dietary adherence was assessed post-intervention, via a self-report item that was scored on a VAS scale ranging from 0 (no adherence) to 100 (very strong adherence). In addition, number of minutes of exercise per week during the intervention was assessed by self-report. For the Think Slim condition, EMA adherence was assessed by analysing the number of reported non-eating moments and eating events, and the number of CBT feedback messages received during the intervention.

\section{Procedure}

Five phases can be distinguished in the present study (see Fig. 1 and Boh et al., 2016). The analyses presented in this paper only focus on the acute effects of the intervention, by comparing post-intervention scores with baseline scores.

Allocation and training phase. Candidates applied by completing an on-line screening questionnaire. After obtaining informed consent, all screened applicants who met the inclusion criteria were sent a technical manual of the iPhone app, and were invited to a technical training for the app (1 day), to make sure the app was working, and was understood by participants. After completing the technical training, participants were randomised into the Think Slim condition or the self-guided diet-only condition. Randomization was stratified by age group (18-30; 31-40; 41-50; 51-60) and gender, and the researcher was blinded for the conditions during randomization.

Baseline measurements (1 week). This week was identical for both conditions. During this week, participants were instructed to obtain supervised measurements of height and weight, and to complete a battery of self-report measures (CCQ, DEBQ, EDE-Q, BSI, SLSC) on-line. In addition, EMA measurements were obtained during the entire week.

Intervention phase (6 weeks). Participants of both conditions received their allocated intervention (Think Slim, or the self-guided diet-only control; see Interventions for more details). Contact with researchers and with the research staff was minimal and was, except for a brief motivational phone call during the third week of the intervention, only related to technical difficulties with the app. So, participants in the self-guided diet-only control condition did not conduct EMA during this phase.

Post-intervention measurements (1 week). After the intervention, there was a second measurement week, which was identical to the first measurement week (baseline). After completing the post-intervention measurements, participants received a voucher of $€ 50$,for participation.

Follow-up phase. During the follow-up phase, participants in the self-guided diet-only control condition received the Think Slim intervention. Participants in the Think Slim condi- 
tion were allowed to continue dieting on their own, and could still enter eating events in the app, but no longer received signal-contingent prompts or web-based intervention sessions. Additional measurements of BMI were obtained and participants filled in the questionnaire battery at 12-weeks and 1-year post-intervention.

\section{Interventions}

\section{Self-guided diet-only control condition}

Participants in the self-guided diet-only control condition were asked to select and adhere to a calorie-restrictive diet of their choice, and to develop and follow an exercise plan. Except for one motivational phone call after the third week of the intervention phase, the diet and exercise plan was entirely self-guided. The self-guided diet-only condition received no further intervention except for two separate on-line sessions during which a diet and an exercise plan were chosen by participants. App functionality for the self-guided diet-only condition was disabled during the intervention phase.

\section{Think Slim intervention condition}

Participants of the Think Slim intervention were also asked to select and adhere to a calorierestrictive diet and exercise plan of their choice, identical to the self-guided diet-only control condition. On top of that, the Think Slim intervention consisted of web-based treatment sessions and an iPhone app, which were designed to work in conjunction: app modules were only activated after certain treatment sessions were completed. In addition, participants conducted EMA throughout the intervention, to provide input for the individually tailored intervention. The content of the app modules and treatment sessions are described in full detail in Boh et al. (2016).

Web-based treatment sessions. The web-based treatment consisted of ten sessions in which participants learned the principles of CBT. The first five sessions were provided during the first week of the intervention phase, whereas the remaining five sessions were provided at the end of every remaining intervention week. Participants were instructed to complete every session. The themes of the sessions were: (1) psycho-education and goal setting, (2) developing skills to deal with dietary temptations, (3) teaching participants to identify and challenge dysfunctional cognitions and thinking errors that would result in undesired eating behaviour and construct functional alternative cognitions, (4) tackling self-esteem and body image, and (5) creating a relapse prevention plan. Lists of dietary goals, motivation, most relevant dysfunctional cognitions, and reasons for losing weight were created by participants and available for reviewing in the app.

Treatment via the app. The app consisted of several modules. Firstly, the passive feedback module compiled EMA data into charts depicting how locations, activities and emotions / states were related to food desire and intake. So, this module provided possibilities for 
self-monitoring. Secondly, the active feedback module provided CBT-based feedback messages. This was done whenever the app detected that it was highly likely that a high-caloric eating event would occur afterwards. The app relied on a decision tree algorithm to detect for each participant when to provide these messages (for more detail, see: Boh et al., 2016; Spanakis et al., 2016). Finally, from the second week until the end of the intervention phase, the thought evaluation module facilitated participants to identify and evaluate dysfunctional cognitions, and formulate functional alternative cognitions. Participants were first presented with a series of open-ended socratic questions. Such questions stimulate a process by which the client explores different facets of a dysfunctional cognition on his/her own, while being steered in the right direction (e.g., "Will this thought help me achieve my weight loss goals?"; Overholser, 2010). Both the dysfunctional cognition and the functional alternative were then scored on believability using VAS items. Evaluated thoughts were stored for later review in the app. Additionally, to expand and increase the relevance of the daily life intervention prompts, participants were now also asked to report dietary temptations, and would receive CBT-based feedback whenever such a dietary temptation was reported.

\section{Data analysis}

First, potential baseline demographic differences between conditions were tested. Then, compliance with the diet and exercise plan and EMA protocol was examined. Foods from the 20-icon overview used in the EMA protocol were divided into either high-caloric, or healthier option foods, based on a classification used in an earlier study (Boh et al., 2016).

Next, the hypotheses about the effectiveness of the Think Slim intervention were investigated. Scores for the main (BMI) and secondary (CCQ, DEBQ, EDE-Q, BSI and SLSC) outcome measures were entered in 2 (time: baseline vs. post-intervention) $\times 2$ (condition: think slim vs control) ANOVAs. In addition, for the Think Slim condition, the CCQ was scored at eight time points throughout the trial (at baseline, as part of the web-based CBT sessions, and post-intervention), and each question of the CCQ was entered separately in repeatedmeasures ANOVAs, to test the effect of time.

To test whether treatment compliance was related to treatment success, change scores of $\mathrm{BMI}$, and of other outcome measures for which a significant time $\times$ condition interaction was found, were correlated with three measures of study compliance (percentage of completed signal-contingent prompts, number of event-related samples, self-report measures of diet and exercise plan adherence). In addition to BM change scores, it was opted to only include outcome measures with significant time $\times$ condition interaction, as this was most closely in line with the goal to focus on differences between Think Slim and the control condition. For these analyses, post-intervention minus baseline change scores were calculated for BMI, and all other outcome measures with a significant interaction effect.

For the EMA data analyses, seven participants were excluded due to not conducting EMA during the baseline measurement week, post-intervention measurement week, or both 
(Think Slim: $n=3$, self-guided diet-only control condition: $n=4$ ). So, a total of 89 participants were included in the analyses. Note that only signal-contingent prompts and eating events were analysed, as there were very few reported dietary temptations (see Table 1). Identical to an earlier study (Boh et al., 2016), EMA-obtained cognitions were divided into: neutral (dinnertime, energy), dysfunctional (emotional, social, control, practical, incorrect reasoning) and healthy intention. Frequency of occurrence of cognitions for each of the categories during the baseline and post-intervention weeks was summed per participant, separately for high-caloric and healthier option eating events. The resulting frequency scores were entered in 2 (time) $\times 2$ (condition) $\times 2$ (assessment: high-caloric eating events, healthier option eating events) ANOVAs. Greenhouse-Geisser corrections were performed where applicable. Emotions obtained via EMA during the baseline and post-intervention measurement weeks were divided into negative (sad / gloomy, tense / stressed, angry / annoyed and anxious / scared), and positive (calm / relaxed and cheerful / happy). In addition, effects on the states of boredom and being tired were analysed. Emotion and state ratings were averaged separately per category and per assessment type (i.e., signal-contingent, high-caloric eating event, healthier eating event) for each participant. A score of zero was considered to reflect a lack of that emotion or state, and zeros were therefore also included in the average. The resulting average ratings were then entered in 2 (time: baseline, post-intervention) $\times 2$ (condition: Think Slim, control) $\times 3$ (assessment: high-caloric eating events, healthier option eating events, non-eating moments) ANOVAs. All analyses were performed in SPSS v. 23.0 (SPSS Inc., USA). Reported results follow the CONSORT guidelines for trial reporting (Moher et al., 2010).

\section{RESULTS}

\section{Sample and baseline characteristics}

Demographic characteristics of the sample: Figure 1 represents the participant flow from recruitment to analysis. In total 300 participants were screened, of which 148 were allowed to participate. Of those 148, 105 finished the intervention. A further 9 participants were excluded from the analyses (see Participants and recruitment), so the final sample consisted of 96 participants. Table 1 contains demographic statistics per condition. There were no differences between conditions for any of the included statistics.

Baseline values of the outcome measures: See Table 3 for means and standard deviations of the baseline values. There were no significant differences in baseline BMI between the Think Slim intervention condition and the self-guided diet-only control condition, $t(94)=1.19$, $p=0.24,95 \% \mathrm{Cl}=(-0.73,2.92)$. At baseline, participants in the Think Slim intervention condition scored significantly higher on the EDE-Q weight concern subscale, $t(94)=2.00, p=.048$, $95 \% \mathrm{Cl}=(0.0004,0.90)$, and significantly higher on the SLSC self-liking subscale, $t(94)=2.25$, 
Table 1. Demographics of the Think Slim and control conditions.

\begin{tabular}{|c|c|c|c|c|}
\hline \multirow[b]{2}{*}{ Demographic } & \multirow{2}{*}{$\frac{\text { Think Slim }}{n}$} & \multirow{2}{*}{$\frac{\text { Diet-only control }}{n}$} & \multicolumn{2}{|c|}{ Comparison } \\
\hline & & & $x^{2}$ & $\mathbf{p}$ \\
\hline Gender & & & $<.0001$ & .99 \\
\hline Male & 10 & 9 & & \\
\hline Female & 36 & 41 & & \\
\hline Employment ${ }^{1}$ & & & 0.87 & .65 \\
\hline Unemployed & 8 & 6 & & \\
\hline Student & 3 & 2 & & \\
\hline Working & 35 & 41 & & \\
\hline Education ${ }^{1}$ & & & $<.0001$ & .99 \\
\hline No degree & 0 & 0 & & \\
\hline Lower & 9 & 9 & & \\
\hline Intermediate & 14 & 14 & & \\
\hline Higher & 17 & 18 & & \\
\hline University & 6 & 7 & & \\
\hline Nationality & & & 3.11 & .21 \\
\hline Dutch & 44 & 49 & & \\
\hline Belgian & 2 & 0 & & \\
\hline \multirow[t]{2}{*}{ German } & 0 & 1 & & \\
\hline & $M(S D)$ & $M(S D)$ & $t(94)$ & $p$ \\
\hline Age & $44.7(11.26)$ & $44.5(10.5)$ & 0.07 & .95 \\
\hline
\end{tabular}

$p=.03,95 \% \mathrm{Cl}=(0.29,4.71)$. There were no significant baseline differences between conditions for any of the other outcome measures, all $t(94)<1.59$, all $p>.12$.

\section{Compliance}

Table 2 contains statistics of compliance, including comparisons between conditions, for post-intervention self-reported diet and exercise adherence, EMA signal-contingent prompt compliance, and eating events reported at baseline and post-intervention. In addition, for Think Slim, EMA signal-contingent prompts, eating events, and number of CBT feedback messages received during the intervention are included. Overall, participants were moderately compliant with completing signal-contingent prompts at baseline (Think Slim participants completed $53.95 \%$ of signal-contingent assessments; control condition participants completed $59.81 \%$ ), and the frequency of reported eating events was similar to previous EMA studies (Boh et al., 2016; Tomiyama, Mann, \& Comer, 2009). Note that, from baseline to post-intervention, there was an overall reduction in percentage of completed signal- 


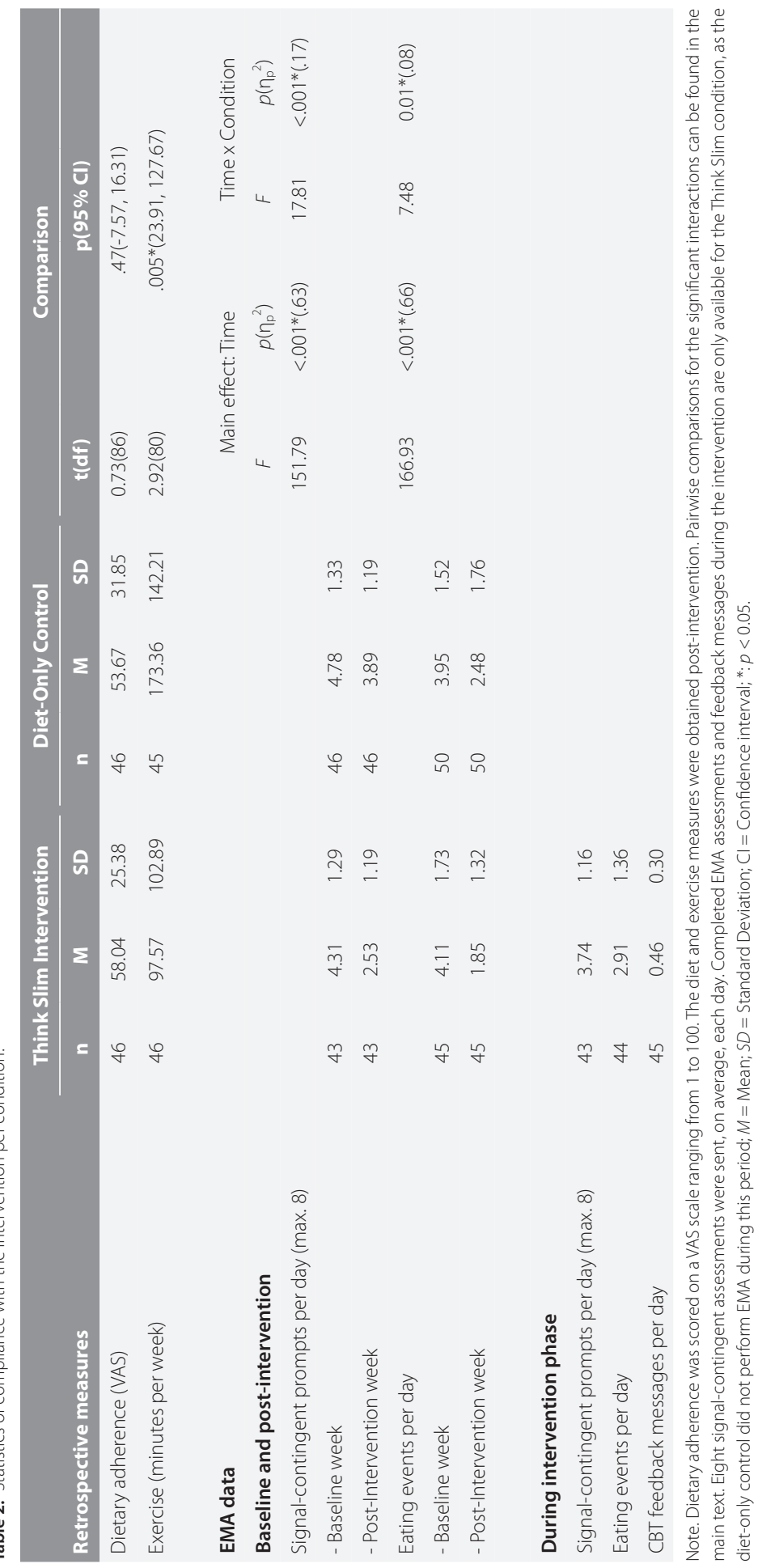


contingent prompts, $t(88)=11.17, p<.0001,95 \% \mathrm{Cl}=(13.49,19.33)$, and an overall reduction in reported eating events, $t(88)=12.39, p<.0001,95 \% \mathrm{Cl}=(10.90,15.06)$. Furthermore, for the signal-contingent prompts and eating events, there were significant time $\times$ condition interaction effects. Further inspection showed that the control condition completed a (trend-)significantly higher percentage of signal-contingent prompts, both at baseline, $t(87)=1.78, p=0.08,95 \% \mathrm{Cl}=(-12.96,0.71)$, and post-intervention, $t(87)=5.75, p<.0001$, $95 \% \mathrm{Cl}=(11.45,23.55)$, which implies that the difference between conditions was larger post-intervention than at baseline. The control condition also reported trend-significantly more eating events post-intervention, $t(93)=1.88, p=.06,95 \% \mathrm{Cl}=(-8.99,0.24)$, but not at baseline, $t(93)=0.48, p=0.63,95 \% \mathrm{Cl}=(-3.64,5.96)$. The post-intervention obtained measures for dietary adherence and exercise per week during the intervention phase indicated that participants in the control condition reported significantly more exercise minutes, but there was no significant difference between conditions for dietary adherence.

\section{Outcomes of the intervention}

Table 3 contains statistics for BMI and the self-report questionnaire measures.

BMI. Participants in both conditions lost significant weight, as is apparent from the main effect of time. However, contrary to expectations, the weight loss was not significantly larger in the Think Slim condition than in the control condition, as is apparent from the nonsignificant time $\times$ condition interaction.

CCQ. As predicted, there was a significant time $\times$ condition interaction. For the Think Slim intervention condition, but not for the self-guided diet-only control condition, belief in dysfunctional cognitions decreased significantly from baseline to post-intervention. In addition, it was shown in a repeated measures analysis of the Think Slim intervention condition that participants scored significantly lower over time on each of the 9 separate questions included in the CCQ, all $F(86)>8.27$, all $p<.001$. See Figure 2 for results and an overview of when the CCQ was administered for the Think Slim condition.

$D E B Q$. In line with the hypothesis, there were significant time $\times$ condition interactions for all three of the DEBQ subscales. Further exploration indicated that scores of participants in the Think Slim intervention condition decreased significantly on the Emotional Eating and External Eating subscales, and increased significantly on the Restrained Eating subscale, whereas participants in the self-guided diet-only control condition showed a trendsignificant increase on the Restrained Eating subscale only, but no significant changes on the other two subscales. 


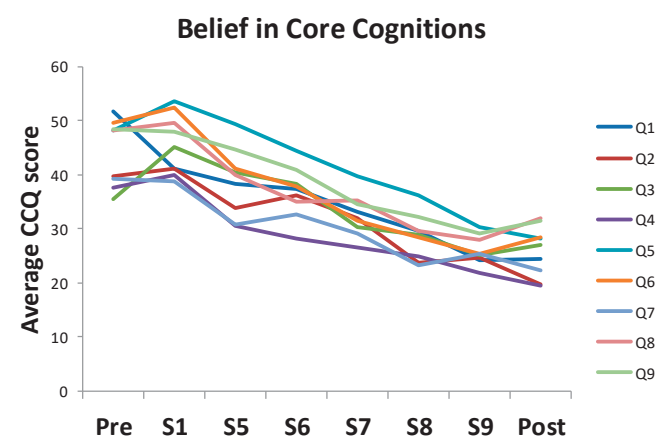

Figure 2. Belief in core cognitions for all assessment occasions of the Think Slim condition.

Note. The $y$-axis represents VAS scores of the CCQ questionnaire (ranging from 0 , no belief, to 100 , very strong belief). The $x$-axis represents the moment of assessment; baseline ('pre'), sessions 1, 5, 6, 7, 8, and 9, and post-intervention ('post'). Each line represents a separate question of the CCQ (from Q1 to Q9). These are:

Q1: When I've earned it, I can reward myself with nice food.

Q2: When I feel bad or tense, eating something I like is a good way to feel better.

Q3: When I'm bored, then eating something I like is a good way to get rid of the boredom.

Q4: When I've eaten more than I planned to, my day is ruined and I might as well continue eating.

Q5: When I start snacking, I cannot stop.

Q6: When everyone is eating something nice, I have to join them.

Q7: When I am offered something nice, it is rude to turn down the offer; I have to eat it.

Q8: When I have food I like at home, I have to eat it.

Q9: When I desire something I like, I have to eat it.

EDE-Q. There were significant main effects of time for all of subscales. Contrary to expectations, none of the time $\times$ condition interaction effects were significant. So, both conditions improved equally in self-reported eating disorder psychopathology.

SLSC. Contrary to expectations, both conditions improved in self-reported self-liking from baseline to post-intervention. There were no significant time $\times$ condition interaction effects. For self-competence, none of the effects reached statistical significance.

$B S I$. None of the main effects or interaction effects were significant, indicating participants did not significantly change in levels of self-reported psychiatric symptoms.

\section{EMA Data}

Descriptives for the EMA data can be found in Table 4a, whereas comparisons between Think Slim and the self-guided diet-only control can be found in Table $4 \mathrm{~b}$.

Cognitions. There was a trend-significant time $\times$ condition $\times$ assessment type interaction for dysfunctional cognitions. To investigate this effect, further time $\times$ condition ANOVAs were conducted separately for each assessment type. In line with expectations, there was a trend-significant time $\times$ condition interaction for high-caloric eating events, $F(87)=3.91$, $p=0.051, \eta_{p}^{2}=.04$, indicating that the improvement was larger in Think Slim than in the control condition. Pairwise comparisons between baseline and post-intervention measurement at high-caloric eating events indicated a significant reduction in dysfunctional cognitions for both Think slim, $t(42)=5.22, p<0.001,95 \% \mathrm{Cl}=(1.31,2.97)$ and the control 


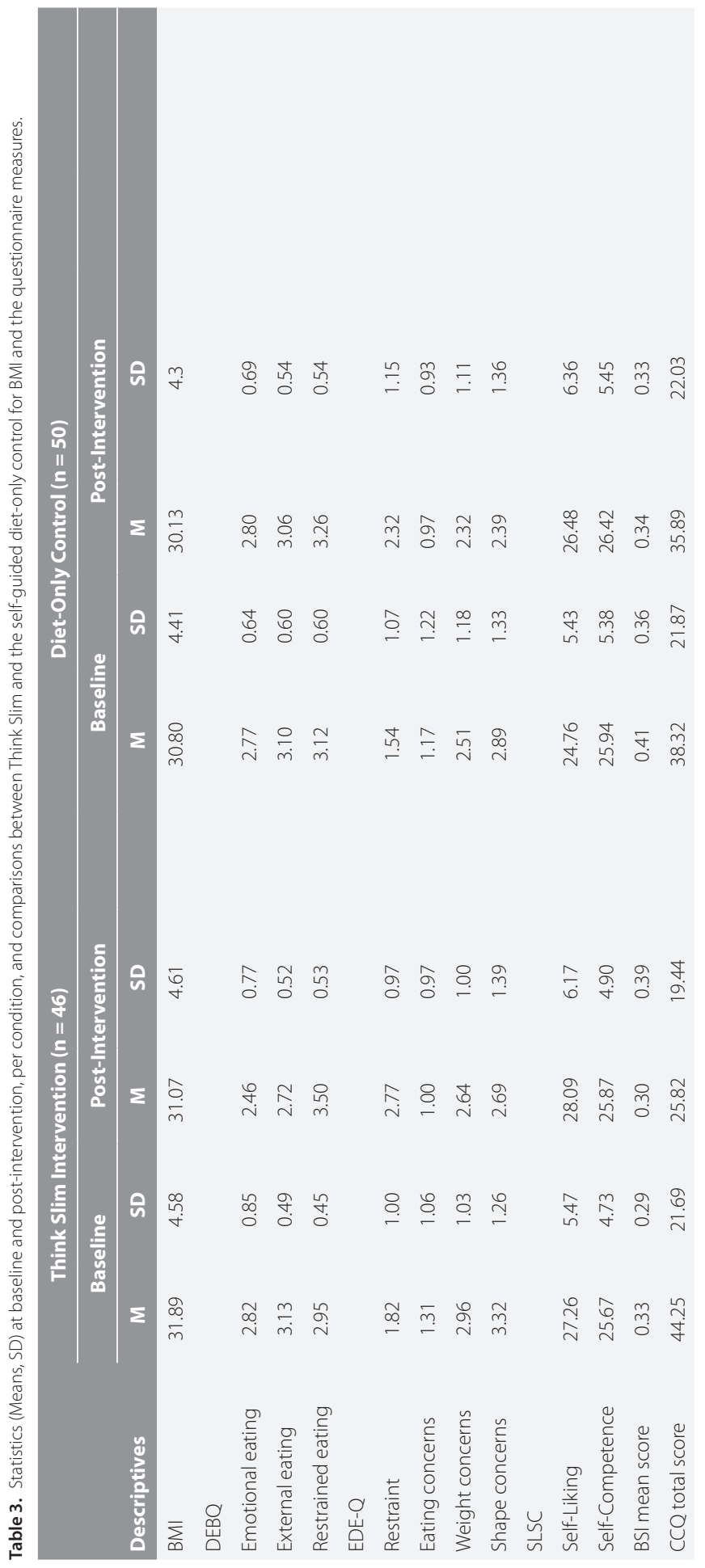




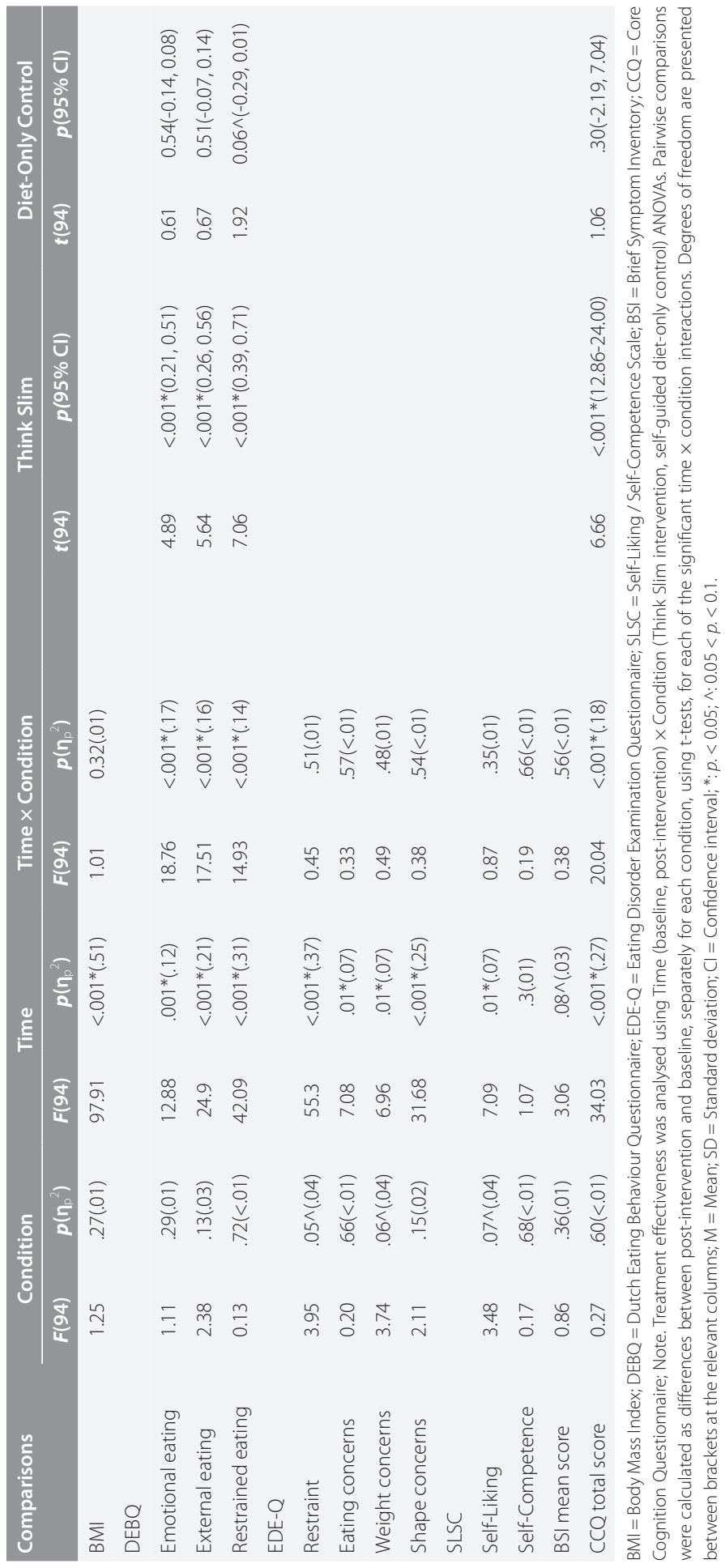


Table 4a. Descriptives (Means, SDs) presented at baseline and post-intervention, per condition.

\begin{tabular}{|c|c|c|c|c|c|c|c|c|c|c|c|c|}
\hline \multirow[b]{4}{*}{ Type of sample } & \multicolumn{12}{|c|}{ Think Slim ( $n=43)$} \\
\hline & \multicolumn{4}{|c|}{ Healthier Option } & \multicolumn{4}{|c|}{ High-Caloric } & \multicolumn{4}{|c|}{ Signal-Contingent } \\
\hline & \multicolumn{2}{|c|}{ Baseline } & \multicolumn{2}{|c|}{$\begin{array}{c}\text { Post- } \\
\text { Intervention }\end{array}$} & \multicolumn{2}{|c|}{ Baseline } & \multicolumn{2}{|c|}{$\begin{array}{c}\text { Post- } \\
\text { Intervention }\end{array}$} & \multicolumn{2}{|c|}{ Baseline } & \multicolumn{2}{|c|}{$\begin{array}{c}\text { Post- } \\
\text { Intervention } \\
\end{array}$} \\
\hline & $\mathbf{M}$ & SD & M & SD & M & SD & M & SD & $\mathbf{M}$ & SD & M & SD \\
\hline \multicolumn{13}{|l|}{ Cognitions } \\
\hline - Neutral & 16.88 & 6.46 & 9.53 & 6.27 & 3.19 & 2.72 & 1.12 & 1.79 & & & & \\
\hline - Dysfunctional & 0.35 & 0.69 & 0.02 & 0.15 & 2.23 & 2.83 & 0.09 & 0.36 & & & & \\
\hline - Healthy Intention & 2.26 & 2.88 & 0.65 & 0.20 & 0.21 & 0.71 & 0.02 & 0.15 & & & & \\
\hline \multicolumn{13}{|l|}{ Emotions and states } \\
\hline - Negative & 0.19 & 0.22 & 0.22 & 0.37 & 0.25 & 0.34 & 0.16 & 0.47 & 0.23 & 0.23 & 0.28 & 0.43 \\
\hline - Positive & 3.91 & 2.13 & 3.53 & 2.67 & 3.68 & 2.54 & 2.21 & 2.77 & 3.70 & 2.15 & 3.40 & 2.59 \\
\hline - Bored & 0.15 & 0.31 & 0.02 & 0.13 & 0.30 & 0.69 & 0.21 & 1.31 & 0.27 & 0.45 & 0.07 & 0.17 \\
\hline - Tired & 1.75 & 1.27 & 1.02 & 1.11 & 1.79 & 1.52 & 0.95 & 2.15 & 2.15 & 1.38 & 1.45 & 1.30 \\
\hline \multirow[t]{4}{*}{ Number of eating events } & 22.74 & 9.50 & 11.74 & 8.37 & 6.07 & 4.27 & 1.23 & 1.84 & & & & \\
\hline & \multicolumn{12}{|c|}{ Self-guided diet-only control $(n=46)$} \\
\hline & \multicolumn{4}{|c|}{ Healthier Option } & \multicolumn{4}{|c|}{ High-Caloric } & \multicolumn{4}{|c|}{ Signal-Contingent } \\
\hline & \multicolumn{2}{|c|}{ Baseline } & \multicolumn{2}{|c|}{$\begin{array}{c}\text { Post- } \\
\text { Intervention }\end{array}$} & \multicolumn{2}{|c|}{ Baseline } & \multicolumn{2}{|c|}{$\begin{array}{c}\text { Post- } \\
\text { Intervention }\end{array}$} & \multicolumn{2}{|c|}{ Baseline } & \multicolumn{2}{|c|}{$\begin{array}{c}\text { Post- } \\
\text { Intervention }\end{array}$} \\
\hline Type of sample & $M$ & SD & $M$ & SD & M & SD & $M$ & SD & $M$ & SD & $M$ & SD \\
\hline \multicolumn{13}{|l|}{ Cognitions } \\
\hline - Neutral & 16.46 & 5.72 & 10.76 & 6.69 & 4.09 & 3.87 & 2.20 & 2.38 & & & & \\
\hline - Dysfunctional & 0.43 & 0.93 & 0.11 & 0.43 & 2.04 & 2.29 & 0.85 & 1.35 & & & & \\
\hline - Healthy Intention & 1.46 & 1.78 & 0.74 & 1.18 & 0.17 & 0.49 & 0.15 & 0.36 & & & & \\
\hline \multicolumn{13}{|l|}{ Emotions and states } \\
\hline - Negative & 0.26 & 0.38 & 0.34 & 0.57 & 0.26 & 0.43 & 0.25 & 0.57 & 0.26 & 0.36 & 0.32 & 0.54 \\
\hline - Positive & 3.63 & 1.66 & 3.11 & 1.96 & 3.60 & 2.15 & 2.50 & 2.08 & 3.43 & 1.58 & 3.24 & 1.85 \\
\hline - Bored & 0.19 & 0.40 & 0.16 & 0.47 & 0.29 & 0.56 & 0.31 & 0.60 & 0.25 & 0.48 & 0.18 & 0.38 \\
\hline - Tired & 1.72 & 1.49 & 1.58 & 1.75 & 1.94 & 2.18 & 1.43 & 1.89 & 2.11 & 1.51 & 2.00 & 1.75 \\
\hline Number of eating events & 20.93 & 8.43 & 13.98 & 9.91 & 6.72 & 4.64 & 3.37 & 3.03 & & & & \\
\hline
\end{tabular}

Note. Eating events are presented per week. Emotions and states are presented as average ratings (provided on VAS items ranging from 0 to 10) with scores of value zero also included. Cognitions are presented as frequencies; $M=$ Mean; SD = Standard Deviation.

condition, $t(45)=4.64, p<0.001,95 \% \mathrm{Cl}=(0.68,1.71)$. For the healthier option eating events, no time $\times$ condition interaction was observed, $F(87)<0.001, p>0.99$.

Emotions and states. Contrary to expectations, there were no significant time $\times$ condition $\times$ assessment interactions, indicating that the Think Slim intervention did not lead to a larger reduction in emotions or states reported immediately before high-caloric eating than the control condition. 
Table 4b. Comparisons between Think Slim and the diet-only control, for the EMA data on cognitions, emotions and states, and eating events.

\begin{tabular}{|c|c|c|c|c|c|c|c|c|}
\hline \multirow[b]{2}{*}{ Type of sample } & \multicolumn{2}{|c|}{ Time } & \multicolumn{2}{|c|}{ Time $\times$ Assessment } & \multicolumn{2}{|c|}{ Time $\times$ Condition } & \multicolumn{2}{|c|}{$\begin{array}{c}\text { Time } \times \text { Condition } \\
\times \text { Assessment }\end{array}$} \\
\hline & $\mathbf{F}$ & $p\left(n_{p}{ }^{2}\right)$ & $\mathbf{F}$ & $p\left(n_{p}^{2}\right)$ & $\mathbf{F}$ & $p\left(n_{p}^{2}\right)$ & $\mathbf{F}$ & $p\left(n_{p}^{2}\right)$ \\
\hline \multicolumn{9}{|l|}{ Cognitions } \\
\hline - Neutral & 93.76 & $<.0001^{*}(.52)$ & 33.96 & $<.0001 *(.28)$ & 1.09 & $.30(.01)$ & 0.90 & $.35(.01)$ \\
\hline - Dysfunctional & 57.32 & $<.0001^{*}(.40)$ & 29.27 & $<.0001^{*}(.25)$ & 3.21 & $.08 \wedge(.04)$ & 3.63 & $.06 \wedge(.04)$ \\
\hline - Healthy Intention & 25.26 & $<.0001^{*}(.23)$ & 22.98 & $<.0001^{*}(.21)$ & 4.36 & $.04 *(.05)$ & 2.69 & $.11(.03)$ \\
\hline \multicolumn{9}{|c|}{ Emotions and states } \\
\hline - Negative & 0.22 & $.64(<.01)$ & 2.99 & $.06 \wedge(.03)$ & 0.48 & $.49(.01)$ & 0.31 & $.73(<.01)$ \\
\hline - Positive & 16.88 & $<.0001(.16)$ & 11.54 & $<.0001(.12)$ & 0.12 & $.73(<.01)$ & 0.62 & $.47(<.01)$ \\
\hline - Bored & 4.36 & $.04 *(.05)$ & 0.77 & $.41(<.01)$ & 2.05 & $.16(.02)$ & 0.03 & $.91(<.01)$ \\
\hline - Tired & 16.07 & $<.0001^{*}(.16)$ & 0.89 & $.39(.01)$ & 3.96 & $.05^{\wedge}(.04)$ & 0.24 & $.74(<.01)$ \\
\hline Eating Events & 166.93 & $<.0001^{*}(.66)$ & 24.89 & $<.0001^{*}(.22)$ & 7.48 & $.01^{*}(.08)$ & 1.70 & $.20(.02)$ \\
\hline
\end{tabular}

Note. $n=89$. EMA data were analysed using Time (baseline, post-intervention) $\times$ Condition (Think Slim intervention, self-guided dietonly control) $\times$ Assessment ANOVAs. Assessment had two levels for cognitions: high-caloric eating events and healthier-option eating events, whereas for emotions one additional level was included: non-eating moments. Eating events represent sums of icon selections in the app, comparing baseline and post-intervention. Multiple icon selections were possible for each eating event EMA assessment. Cognitions are presented as sums of frequency of occurrence, averaged separately for high-caloric and healthier option eating events, and per condition. So, the sum of eating events differs from the sum of the reported cognitions, as the latter were reported once per EMA assessment.

$*: p<0.05$

$\wedge: 0.1>p \geq 0.05$

Correlations of several outcome measures with treatment compliance. See Table 5 for an overview of relevant statistics. In line with expectations, higher levels of self-reported dietary adherence were significantly associated with a greater reduction in BMI, from baseline to post-intervention. In addition, higher scores on self-reported dietary adherence were significantly associated with a greater decrease in belief in dysfunctional cognitions (CCQ) and increase in restrained eating (DEBQ), and trend-significantly associated with a greater decrease in emotional eating (DEBQ), from baseline to post-intervention. Furthermore, for the Think Slim condition, participants who reported more eating events also believed significantly less in dysfunctional cognitions (CCQ) and scored lower on emotional eating (DEBQ), from baseline to post-intervention.

\section{DISCUSSION}

The present randomized controlled trial (RCT) investigated whether a 6-week ecological momentary intervention (EMI) based on cognitive behavioral therapy (CBT) for weight loss (Think Slim) with no therapist support resulted in more weight loss, greater reduction in 
Table 5. Correlations (Pearson's $r$ ) between measures of compliance and change scores (post-intervention minus baseline) for the outcome measures.

\begin{tabular}{|c|c|c|c|c|c|c|}
\hline Both conditions combined & $=$ & 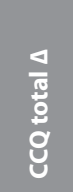 & 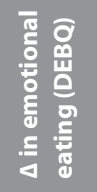 & 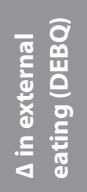 & 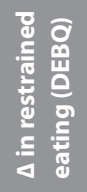 & $\stackrel{\unlhd}{\sum_{m}}$ \\
\hline Reported dietary adherence & 92 & $-.35^{*}$ & $-.19 \wedge$ & -.12 & $.21^{*}$ & $-.21 *$ \\
\hline Minutes of exercise per week & 91 & 0.17 & 0.11 & $0.22^{*}$ & $-0.26^{*}$ & 0.02 \\
\hline \multicolumn{7}{|l|}{$\begin{array}{l}\text { Think Slim condition } \\
\text { (during intervention) }\end{array}$} \\
\hline CBT feedback messages & 45 & .16 & -.04 & -.19 & .09 & .04 \\
\hline$\%$ Completed signal-contingent prompts & 43 & -.05 & -.22 & -.06 & -.10 & .01 \\
\hline Total reported eating events & 44 & $-.43^{*}$ & $-.60^{*}$ & -.15 & .21 & -.08 \\
\hline
\end{tabular}

$C C Q=$ Core Cognition Questionnaire; DEBQ = Dutch Eating Behaviour Questionnaire; BMl = Body Mass Index; Note. Only outcome measures with a significant interaction were included in these analyses. Scores for dietary adherence and minutes of exercise per week were obtained post-intervention and pertain to the intervention phase. For Think Slim, performance on three EMA measures during the intervention was also included. Change scores were calculated as post-intervention minus baseline scores, and averaged per condition. A negative change-score was expected for all measures except for DEBQ restrained eating. So, a negative correlation can generally be interpreted as more improvement with more adherence.

*: $p<0.05$

$\wedge: 0.1<p \geq 0.05$

belief in dysfunctional cognitions, and greater improvements on dysfunctional eating styles, eating disorder psychopathology, diverse psychiatric symptoms, and self-esteem than a selfguided diet-only control. Participants in both conditions showed a statistically significant, but modest, reduction in BMI. Unexpectedly, this reduction was not significantly larger for the Think Slim condition. Similarly, participants in both conditions reported a similar decline in eating disorder psychopathology and self-liking. Interestingly, only participants in the Think Slim condition improved on the measures of dysfunctional cognitions and dysfunctional eating styles. These are factors that are directly targeted in CBT. Some corroborating evidence was found in the EMA data, in that the reduction of dysfunctional cognitions for high-caloric eating events was larger for the Think Slim condition than the control condition. However, contrary to the results of the DEBQ emotional eating subscale, the EMA data indicated that Think Slim participants did not become less emotionally negative prior to high-caloric eating events than participants in the control condition. Also of note is that improvement on several of the outcome measures (i.e., BMl, dysfunctional cognitions, emotional eating and restrained eating) was positively associated with treatment compliance.

The BMI reduction achieved in the present study is roughly comparable to other CBT for weight loss trials (Cooper et al., 2010; Stahre et al., 2005; Werrij et al., 2009). Other weight loss trials also found that the included treatment conditions lost comparable weight in the acute phase (Werrij et al., 2009; Cooper et al., 2010). However, two trials did find a selective weight reduction for the CBT condition (Stahre et al., 2005; 2007). It can be argued that the clinical 
relevance of these weight losses (0.82 BMI for Think Slim; $0.67 \mathrm{BMI}$ for the control) is relatively low. However, the treatment period was quite short ( 6 weeks). Possibly, providing treatment for longer periods would lead to more weight loss and to weight loss maintenance (Franz et al., 2007; Middleton et al., 2012).

An important outcome measure was the belief in eating-related dysfunctional cognitions, which is central to CBT. Teaching overweight and obese individuals how to deal with thoughts and emotions was suggested to help long-term weight loss maintenance (Byrne, 2002; Carter \& Jansen, 2012; Hill, Wyatt, Phelan, \& Wing, 2005; Karhunen et al., 2012). In line with expectations, from baseline to post-intervention, participants in the Think Slim condition were less likely to believe in eating-related dysfunctional cognitions, than those in the control condition. This result differs from Werrij et al. (2009), in which both conditions reported reduced beliefs in dysfunctional cognitions from baseline to post-intervention. Possibly, the relatively more intensive control condition of that trial, which included nutrition education, food diaries and cooking classes, was sufficient to reduce belief in dysfunctional cognitions, whereas the present trial's self-guided diet-only control was not. Note that in Werrij et al. (2009) only the CBT condition showed weight-loss maintenance though. It does give hope for the long-term results of the present trial.

In addition, Think Slim, but not the control condition, led to significantly less self-reported emotional and external eating. These results are in line with another study in which CBT for obesity resulted in a reduction of dysfunctional eating styles (Gade et al., 2014). Contrary to expectations, both conditions improved on self-reported eating psychopathology (EDE$Q)$, self-liking (SLSC), and increased in restrained eating $(D E B Q)$, although the increase in restrained eating was greater for the Think Slim condition. The increase in restrained eating suggests that participants indeed started to try to control their food intake, especially those in the Think Slim condition. Participants did not decrease in general psychiatric symptoms (BSI), but they already scored relatively low at baseline, so a decrease during the intervention was unlikely to occur.

The EMA data of the present trial present a nuanced view of the influence of cognitions, emotions, and states on eating behaviour. The cognition EMA data corroborated the questionnaire data, as only participants in the Think Slim condition reported fewer dysfunctional cognitions post-intervention. However, the frequency of reported dysfunctional cognitions in the present study was very low. So, contrary to expectations, these results do not imply that dysfunctional cognitions play a large role in high-caloric eating behaviour of overweight individuals. It might be that these cognitions were not reported by participants, or were not properly identified by participants despite the (relatively short) CBT training.

It is also important to note that the emotional eating findings in the present study were not corroborated by the EMA data. At all assessment occasions, the highest rated and most often occurring emotions were positive. Most importantly, however, contrary to the reduction in self-reported emotional eating specifically for the Think Slim condition, the EMA data 
did not indicate that participants in the Think Slim condition reported fewer negative emotions prior to high-caloric eating events than those in the control condition. Recent studies investigating self-reported emotional eating have concluded that questionnaire scores may reflect participants' beliefs in the importance of the association between emotions and eating, and may not be specifically related to eating behaviour (Adriaanse, de Ridder, \& Evers, 2011; Boh et al., 2016; Bongers, de Graaff, \& Jansen, 2016; Evers, de Ridder, \& Adriaanse, 2009). The discrepancy between the EMA signal-contingent assessments (which can be obtained on a 2-hourly basis) and the retrospective self-report measures (which ask participants to recall events from several weeks past) could be influenced by memory biases. For example, emotionally salient events are disproportionately often recalled (Schwarz, 2007).

An important limitation in the present study is the overall reduction in EMA compliance, especially in the Think Slim condition. This may have obscured potential differences between conditions, as it is likely that compliance would have been lowest for emotionally intensive moments, during which conducting an EMA assessment would be most intrusive. This issue of treatment compliance may be a more general problem of obesity treatment, as it also occurs for face-to-face obesity treatment (Moroshko, Brennan \& O’Brien, 2011).

Interestingly, two measures of treatment compliance in particular strongly influenced the results. First, analyses of BMI revealed that participants who scored higher on self-reported dietary adherence showed a larger reduction in BMI. This is in line with previous findings that indicate dietary adherence is a crucial factor for achieving weight loss (Sacks et al., 2009). The present study expands those findings from Sacks et al. (2009), as participants who reported higher dietary adherence also demonstrated more improvements on self-reported belief in dysfunctional cognitions (CCQ), scored trend-significantly lower for emotional eating (DEBQ) and significantly higher for restrained eating (DEBQ). So, dietary adherence is not only important for weight loss, but also for eating-related cognitive and emotional improvement. Second, participants in the Think Slim condition who reported more eating events during the intervention phase also improved more on belief in dysfunctional cognitions (CCQ) and emotional eating (DEBQ). Dysfunctional cognitions are specifically identified and evaluated in the app during these EMA-reported eating events. So, this finding illustrates that the thought evaluation module of Think Slim was effective at reducing the belief in such cognitions, relative to how compliant Think Slim participants were with the EMI.

In sum, the present trial investigated a CBT-based EMI to lose weight, and compared it to a self-guided diet-only control condition. Both conditions resulted in statistically significant, but modest BMI reductions, for which the best predictor was dietary adherence. Only the Think Slim condition resulted in a reduction of belief in eating-related dysfunctional cognitions, and reductions in emotional and external eating. The EMA data also indicated fewer dysfunctional cognitions reported post-intervention, but did not corroborate the results of the DEBQ emotional eating scale. However, there was a large drop in EMA compliance, which may have influenced these results. The current weight loss results were modest, but 
comparable to several other CBT for weight loss trials (Cooper et al., 2010; Stahre et al., 2005; 2007; Werrij et al., 2009). As cognitive and behavioural changes were previously suggested to be crucial for long-term weight loss maintenance (Carter \& Jansen, 2012; Hill et al., 2005; Karhunen et al., 2012), and CBT resulted in both cognitive and behavioural improvement and long-term weight loss maintenance in one trial (Werrij et al., 2009), this gives hope for the present trial's long-term results.

\section{ACKNOWLEDGMENTS}

This research was supported by grant 12028 from Technology Foundation STW, National Initiative Brain and Cognition (NIHC), Netherlands Organisation for Scientific Research (NWO) and Philips under the Partnership programme Healthy Lifestyle Solutions to the last author. The authors are grateful to Vincent Kerkhofs for programming the app and website used in the present study, to Michiel Vestjens and Charlie Bonnemayer for programming and maintaining the web-based CBT platform, and to Richard Benning for providing input on the visual design of the app and web-based platform. Also, the authors would like to thank Dorien Blezer and Lianne van Cleef for help with recruitment efforts, participant contact and participant administration. Lastly, the authors thank Jettie Hoonhout for valuable input on the study's design. 


\section{Chapter 6}

General Discussion 

For the present thesis, the goal was to develop an e-Health intervention for weight loss (Think Slim). This intervention was based on the principles of cognitive behavioral therapy (CBT; Beck, 2011) and relied on mobile technology and the internet to deliver CBT in daily life. CBT for obesity assumes that overeating is caused by dysfunctional cognitions that emphasize food intake rather than restraint (Beck, 2007; Jansen \& Mulkens, 2015; Werrij, 2005). The goal of CBT for obesity is to break the vicious cycle of dysfunctional cognitions alternated with overeating through cognitive restructuring and behavior adjustment (Jansen \& Mulkens, 2015; Werrij, 2005). To enable the provision of CBT for obesity at moments when overeating is likely, it was first essential to determine how cognitions, emotions, and high-caloric food desires are related to (over)eating in daily life, and if overweight participants could indeed be distinguished from healthy-weight participants on these factors. To do this, ecological momentary assessment (EMA) was used. For Think Slim, we were mainly interested in CBTrelated variables, so the EMA focused primarily on cognitions, emotions, food desire, and food intake.

\section{RELATING COGNITIONS, EMOTIONS AND FOOD DESIRES WITH FOOD INTAKE VIA EMA}

In the first paper about the EMA study on cognitions, emotions, food desires and food intake, (Chapter 2), the focus was on cognitions and emotions, assessed prior to high-caloric eating, non-high-caloric eating, and randomly throughout the waking day. For cognitions, to briefly reiterate, the CBT model for obesity suggests that dysfunctional cognitions (i.e., thoughts that promote maladaptive behavior, such as overeating) should occur more often for overweight, than for healthy-weight individuals (Beck, 2007; Jansen \& Mulkens, 2015; Werrij, 2005). Previous laboratory research showed that dysfunctional cognitions concerning eating, weight, and shape, played a role in overweight participants' eating behavior (Nauta, Hospers, Jansen, et al., 2000; O'Connor \& Dowrick, 1987). So, it was hypothesized that, in daily life, dysfunctional cognitions would occur more often, and would be more believable, for overweight participants than for healthy-weight participants, at high-caloric eating events. Contrary to expectations, dysfunctional cognitions were reported only sparingly, and overweight participants did not report more dysfunctional cognitions prior to (high-caloric) food intake than healthy-weight participants. In fact, most reported cognitions were related to food desires and the taste of food. The only category that differed between overweight and healthy-weight participants was the 'unrelated' category, with thoughts that were not directly related to food intake (e.g. "I am going to the supermarket", "I don't think anything"). The main conclusion is that, contrary to what the CBT model for obesity suggests, dysfunctional cognitions might not play a large role in overweight individuals' eating behavior in everyday life. However, some caution is warranted. Eating 
behavior is a process of many individual decisions (Wansink \& Cashman, 2007), and so it might be that dysfunctional cognitions play a more prominent role at an earlier stage in the decision process (e.g., when about to buy food, or when deciding to go to a party). In addition, participants in the present EMA study did not receive any training in how to identify dysfunctional cognitions, which is a weakness, because such training is a central component of CBT (Beck, 2011).

The CBT model for obesity also suggests that negative emotions result from dysfunctional cognitions, and influence dieting effectiveness by reducing self-efficacy (Werrij, 2005). A few studies so far have reported a stronger association between negative emotions and eating high-caloric foods for overweight participants than for healthy-weight participants (Appelhans, Whited, Schneider, Oleski \& Pagoto, 2011; Schneider, Appelhans, Whited, Oleski \& Pagoto, 2010). It was therefore hypothesized that in daily life, overweight participants would also report more negative emotions when they were about to eat a high-caloric food than healthy-weight participants. Contrary to what was expected, overweight participants, as compared to healthy-weight participants, had stronger associations between negative emotions and high-caloric eating events, non-high-caloric eating events, and non-eating moments. So, it seems overweight participants were more negative in general, which corroborates previous research (e.g., Faith, Matz, \& Jorge, 2002; Kasen, Cohen, Chen, \& Must, 2008). Note that by far, the most reported emotions were positive, regardless of whether participants were eating or not, and regardless of participant group.

A secondary aim of the emotion assessment of the study presented in Chapter 2 was to investigate whether scores on emotional eating questionnaires were positively associated with negative emotions specifically preceding high-caloric eating events. According to theories of emotional eating (van Strien, Frijters, Bergers, \& Defares, 1986), there should be a stronger association between negative emotions and high-caloric eating, than between negative emotions and non-high-caloric eating or at non-eating moments, for those scoring high on emotional eating questionnaires. However, it has been shown that emotional eating questionnaire scores represent participants' beliefs that emotions and eating behavior are associated, rather than actual associations (Adriaanse, de Ridder, \& Evers, 2011; Bongers, de Graaff, \& Jansen, 2016; Evers, de Ridder, \& Adriaanse, 2009). Therefore, in the present thesis, it was expected that there would not only be a positive association between negative emotions and high-caloric eating, but also between negative emotions and other eating events, and even non-eating moments. This would indicate participants scoring high on emotional eating related questionnaires had a generally elevated negative mood. In line with expectations, it was found that participants scoring high on emotional eating questionnaires reported more negative emotions at any type of assessment. This implies that beliefs about the associations between factors such as emotions and eating may be influenced by memory recall biases such as the recency effect (Schwarz, 2007). 
In a separate paper (Chapter 3), based on the same EMA study, the hypothesis was tested that overweight participants had stronger and more frequent (high-caloric) food desires than healthy-weight participants. It was also tested whether food desires and food intake were more varied for overweight participants than for healthy-weight participants. To expand on previous findings indicating overweight participants had more frequent food desires than healthy-weight participants (Chao et al., 2014), eating events and non-eating moments were investigated separately. It was found that overweight participants indeed had more high-caloric food desires than normal weight participants, but this difference was only found at non-eating moments of assessment. Directly before eating a high caloric food, both conditions desired equally much. Healthy-weight participants, on the other hand, had more food desires for staple foods (i.e., foods primarily eaten during breakfast, lunch and dinner, such as bread or rice), and these desires occurred specifically at eating events. These results are in line with findings that indicate that overweight participants are more susceptible to the rewarding properties of high-caloric food (Davis et al., 2007).

Interestingly, and in line with expectations and prior research findings, it was also found that high-caloric food desires, but not non-high-caloric food desires, were more varied for overweight than for healthy-weight participants, regardless of whether they were about to eat or not. However, surprisingly, and contrary to expectations, this difference was not present for food intake variety: In the present study, overweight and healthy-weight participants reported an equal number of eating events, and reported an equal variety of food consumption. It should be noted that, in the present study, food intake was often not accompanied by a specific food desire. That is, specific food desires occurred only for about $40 \%$ of the eating events, which may have reduced the likelihood of finding group differences. These findings on food intake variety are contrary to some previous research (e.g., Raynor et al., 2005), where overweight participants were found to consume more varied foods than healthy-weight participants.

When interpreting the findings related to food desire and intake, some caution is warranted. It is possible that due to the set-up of the study, in which participant were asked to self-report food desire and intake by selecting foods from an overview of icons representing the most common foods in the Western diet, participants only selected food icons representing the most healthy foods. Due to the nature of the study (assessing food intake in an app, which would be analyzed by researchers), participants may have wanted to create a socially desirable image. In addition, food intake quantity was not measured. So, whereas eating events and food intake variety did not differ, it might be that portion size differences are more important predictors of obesity (Ledikwe, Ello-Martin, \& Rolls, 2005; Rosenheck, 2008). 


\section{CBT FOR OBESITY IN DAILY LIFE: TESTING THE THINK SLIM EMI IN AN RCT}

The Think Slim intervention itself was tested in a randomized controlled trial (RCT), comparing it to a self-guided diet-only control condition. The Think Slim intervention consisted of an iPhone app and a web-based platform. The web-based platform provided CBT sessions to participants, and the app was designed as a tool to facilitate the therapeutic process, and to provide therapy in daily life. For the RCT, it was hypothesized that the Think Slim intervention would result in (1) more weight loss, (2) less belief in, and fewer reported, dysfunctional eating-related cognitions, (3) less general- and eating-related psychopathology, (4) more self-esteem, and (5) less strong positive associations of (negative) emotions with eating behavior, than the diet-only control.

Contrary to expectations, both conditions lost a statistically significant, but clinically modest, amount of weight, with no difference between conditions at the end of the intervention. In addition, both conditions resulted in a reduction in eating disorder psychopathology and an increase in self-liking. Interestingly, and in line with expectations, only participants in the Think Slim intervention, and not those in the diet-only control, believed less in dysfunctional cognitions (e.g., "When I feel bad or tense, eating something I like is a good way to feel better") at the end of the intervention than at baseline. Also, only participants in the Think Slim condition scored lower on questionnaire measures of emotional and external eating, and higher on a questionnaire measure of restrained eating post-intervention compared to baseline. So, whereas BMI reductions were comparable between both conditions, only Think Slim resulted in some CBT-related improvements.

These specific reductions of belief in eating-related dysfunctional cognitions and emotional and external eating behavior were partly corroborated by the EMA data. Participants in the Think Slim condition, but not in the control condition, showed a reduction in dysfunctional cognitions prior to high-caloric eating events. However, unexpectedly, for emotions, no significant differences between Think Slim and the diet-only control condition were found when comparing post-intervention with baseline. So, again, the results with a retrospective questionnaire measure of emotional eating are not in line with those of a measure that arguably assesses emotions and eating behavior more directly and promptly (Adriaanse et al., 2011; Bongers et al., 2016; Evers et al., 2009). Importantly, however, the EMA results should be interpreted with caution, as there was a large reduction in participant compliance from baseline to post-intervention. Moreover, this reduction was larger for the Think Slim condition than for the diet-only control, which was most likely due to being enrolled in the EMA protocol for the duration of the entire intervention (whereas the diet-only condition only performed EMA during the baseline and post-intervention weeks). Possibly, participants were least compliant with the EMA procedure when they were in emotionally intensive situations, as having to complete the EMA questionnaire during such times would be most intrusive. 
Various measures of compliance were also correlated with weight loss, belief in dysfunctional cognitions, and emotional / external eating. For both conditions, dietary adherence and number of minutes of exercise per week were analyzed. Additionally, for the Think Slim condition, percentage of completed EMA non-eating moment assessments, and number of reported eating events were analyzed. It was hypothesized that participants who adhered more to their diet would lose more weight and show greater improvements on self-assessed dysfunctional cognitions, emotional eating, external eating, and restrained eating. In line with previous research (Sacks et al., 2009), self-reported dietary adherence was positively associated with weight loss all participants taken together. Also, dietary adherence was positively associated with reduction in belief in dysfunctional cognitions and increased restrained eating, for both conditions. So, these results expand the findings of Sacks et al (2009), by showing that dietary adherence is not only associated with dietary effectiveness, but also with reductions in belief in dysfunctional cognitions and emotional / external eating.

The acute results for weight loss in the present trial are modest. However, it has previously been argued that targeting cognitions and other antecedents of eating behavior is essential for achieving long-term weight loss maintenance (Carter \& Jansen, 2012; Karhunen et al., 2012). This was also shown in various trials, which found that participants in the CBT for weight loss condition were able to maintain their weight loss at a one-year follow-up measurement, whereas the control conditions regained the weight (Sbrocco, Nedegaard, Stone, \& Lewis, 1999; Stahre \& Hällström, 2005; Stahre, Tärnell, Håkanson, \& Hällström, 2007; Werrij et al., 2009), although not all studies found weight maintenance for the CBT condition (Zafra Cooper et al., 2010). So, for the present trial, it is hypothesized that, at the one-year follow-up measurement (which has not yet taken place at the time of writing), participants in the Think Slim condition will have retained their weight loss, whereas participants in the control condition will have regained their lost weight.

\section{Main conclusions}

1. No support was found for the hypothesis that dysfunctional cognitions occur more often in overweight than healthy-weight participants. In the first study (Chapter 2), dysfunctional cognitions occurred with a low frequency, whereas most reported cognitions were neutral, and related to food desire and food taste. This was corroborated by the second study (Chapter 5), in which also relatively few dysfunctional cognitions were reported by overweight participants. It is likely that dysfunctional cognitions, as assessed frequently throughout the day via EMA, do not play a prominent role in eating behavior. Another possibility, which has not been tested in the present thesis, is that dysfunctional cognitions play an important role at an earlier stage, that precede highcaloric food intake, such as when purchasing the food, when about to go to a party, or when experiencing a dietary temptation. 
2. The CBT model for obesity suggests that negative emotions play an important role in eating behavior, by reducing the effectiveness of dieting. However, no support for this idea was found in the present thesis, as overweight participants were more emotionally negative regardless of whether they were eating or not, compared to healthy-weight participants. So, it is more likely that overweight people are generally more negative than healthy-weight people. This is in line with previous research that found that overweight participants have generally elevated depressive symptomatology (Faith, Fontaine, Baskin, \& Allison, 2007; Kasen, Cohen, Chen, \& Must, 2008).

3. Emotional eating, which is defined as eating high-caloric food in response to negative emotions (van Strien et al., 1986) was suggested as an influential eating style that would explain overeating behavior. In the present thesis (Chapters 2 and 5), however, no support for this idea was found either. Instead, participants scoring high on a questionnaire on emotional eating were also more negative at two other types of assessment moments (i.e., non-high-caloric eating events and non-eating moments). Interestingly, in the second study of the present thesis (Chapter 5), for emotional eating, the EMA data did not corroborate the emotional eating questionnaire data. Whereas participants in the Think Slim condition scored lower on emotional eating post-intervention relative to baseline, the EMA data did not show any reduction in negative emotions prior to high-caloric eating events, which may have been due to differences between these two methods of investigation.

4. In line with prior research (Chao, Grilo, White, \& Sinha, 2014; Delahanty, Meigs, Hayden, Williamson, \& Nathan, 2002; Franken \& Muris, 2005; Ouwehand \& Papies, 2010), it was found that high-caloric food desires occurred more often in overweight participants, with the present research finding that this difference between overweight and healthyweight participants for high-caloric food desires occurred only at pseudorandom assessments (when participants were not eating). In addition, such food desires were more varied than those of healthy-weight participants. So, overweight individuals' high-caloric food desires seem to be more closely tied to non-eating related factors (e.g., more abstract cues in the environment) than those of healthy-weight individuals. CBT for obesity should teach overweight individuals to deal effectively with cognitions and emotions surrounding food desires in the everyday environment that occur at non-eating moments (Carels et al., 2001; Carels, Douglass, Cacciapaglia, \& O'Brien, 2004; McKee, Ntoumanis, \& Taylor, 2014).

5. The Think Slim intervention did not result in more weight loss than a diet-only control. In retrospect, this result might have been due to the relatively short period of the intervention ( 6 weeks). However, Think Slim did achieve (1) a reduction of belief in dysfunctional cognitions, (2) a reduction in the frequency of dysfunctional cognitions reported prior to high-caloric eating, (3) a reduction in self-assessed emotional and external eating, and (4) an increase in self-assessed restrained eating. For the diet-only control condi- 
tion, post-intervention and baseline scores did not differ significantly for any of these variables. Improvements in terms of belief in dysfunctional cognitions and emotional / external eating may be crucial for long-term weight loss maintenance (Karhunen et al., 2012). So, it is hypothesized that the follow-up results of this RCT will show weight maintenance for the Think Slim condition, but not the control condition.

\section{Future research}

The findings described in Chapter 2 suggest that, contrary to expectations, dysfunctional cognitions immediately prior to eating should not be a main target for obesity interventions. Eating-related dysfunctional cognitions might play a more prominent role in overweight people's overeating behavior at earlier stages when deciding to eat or experiencing a dietary temptation (e.g., when deciding to buy snacks, or when about to go to a restaurant). Future research on eating-related cognitions could investigate whether dysfunctional cognitions occur more for overweight participants than for healthy-weight participants these earlier food-related decisions.

It might be, however, that dysfunctional cognitions in general simply do not greatly influence eating behavior. Aspects such as eating habits, which may not have a strong cognitive component, may be a more important focus (Epstein, Temple, Roemmich, \& Bouton, 2009). Therefore, insights into how different factors, such as locations, activities, social circumstances, food desires and emotions affect eating behavior might be more crucial. To accomplish this, the focus of statistical analyses could be on the trajectory from a certain critical time point (such as a dietary temptation) to the time of food intake. By relying on methods such as network analysis (Borsboom \& Cramer, 2013; Spanakis, Weiss, Boh, \& Roefs, 2016), insights can be obtained into how food-related decisions translate into eating behavior, even if such eating behavior is several hours in the future. For example, feeling bad due to a negative life event may negatively influence social activities in the subsequent time period, which may be positively associated with boredom and food desire, leading to a high-caloric eating event. By knowing which variable states precede high-caloric eating, prophylactic treatment can be offered (in the context of daily life).

Next, the compliance of EMA and EMI protocols is always an issue. In Think Slim, participants mostly reported that it was too much hassle to keep completing the EMA questionnaires. Improving the compliance with the EMA protocol should lead to significantly more instances of dietary temptations, food-related decisions, and risky circumstances being assessed. These assessments could then be targeted by the intervention. To do this, future EMls for obesity should consider implementing automated sensor technology, through context-sensitive EMA (csEMA; Intille, 2007). csEMA represents the online analysis of EMA data about participants' movements, physical locations, and internal physiological states, to intervene at critical moments in time, without the input of the participant. So, the quality of csEMA depends on the validity of the instruments that are used to assess the contextual 
factors of interest. For example, by including a GPS sensor into an EMI for obesity, participants no longer have to be asked about their location at certain times throughout the day. Instead, location can automatically be associated with food-related decisions, and dietary temptations. This method is already being used in several EMA systems (Intille, Rondoni, Kukla, Ancona, \& Bao, 2003; Ramanathan et al., 2012), and is easy to implement on mobile devices. Using GPS allows CBT-based feedback messages to warn participants about risky circumstances whenever they enter locations that have a high chance of leading to overeating. Another interesting option is the automatic analysis of facial features to recognize emotional states. However, this method is still under development (Kołakowska, Landowska, Szwoch, Szwoch, \& Wróbel, 2013). In line with this development, the most important consideration for other sensor applications, such as those for heart rate, heart rate variability and skin conductance, is whether such measures reflect food desire / food consumption, or unrelated factors, such as stress and physical activity. Research should therefore focus on increasing the predictive validity of these measures.

\section{In Closing}

It can be concluded that dysfunctional cognitions and negative emotions at high-caloric eating events do not seem to be essential targets of obesity treatment. Most cognitions assessed in daily life were not dysfunctional and did not occur more often in overweight participants. In addition, overweight participants were more negative regardless of whether they were eating or not. The CBT-based EMI for weight loss used in the present thesis did result in a reduction of questionnaire-assessed belief in dysfunctional cognitions and emotional / external eating. These changes may prove useful for weight-loss maintenance at follow-up.

Future research may target earlier eating-related decisions, to elucidate which variables determine when and if one buys high-caloric foods, and when and if one will experience a moment of temptation, to enable intervention at these earlier moments. Also, future research should examine the validity and usefulness of sensor-data for understanding eating-behavior and interventions for weight loss, to alleviate the burden of data-input on participants. 
English Summary 

The central goal of the present thesis was to examine the effectiveness of CBT for obesity in the context of daily life, by means of an EMI, via an iPhone app and a web-based platform. First, the role of cognitions, emotions, food desire and food intake was examined in daily life, via ecological momentary assessment (EMA) and compared between overweight and healthy-weight participants. Second, the EMI for obesity ('Think Slim') was compared to a diet-only control. The main findings of the thesis will be summarized.

Chapter 1 provides an overview of the purpose of the present thesis from a theoretical viewpoint. The majority of overweight people regularly diet, and there are many interventions for weight loss. However, the success rates of dieting and weight loss are generally low, especially in the long term (e.g., Franz et al., 2007; Mann et al., 2007). Most individuals do not achieve the weight losses they hoped for, and eventually regain the lost weight (Zafra Cooper et al., 2010). The cognitive behavioral therapy (CBT) model for obesity postulates that dieting and long-term weight loss may be more effective by targeting dysfunctional thoughts (cognitions) that may cause people to overeat in spite of the negative long-term consequence of weight gain (Carter \& Jansen, 2012; Jansen \& Mulkens, 2015; Jansen, Nederkoorn, Roefs, Martijn, \& Havermans, 2009; Karhunen et al., 2012). Such dysfunctional cognitions (e.g. "When I finish my work, I can reward myself with a snack.") perpetuate a vicious cycle of inadequate weight loss attempts, as they increase negative mood, decrease self-efficacy, self-esteem, and increase concerns about eating, shape and weight, leading to high-caloric eating events. To break this cycle, dysfunctional cognitions are identified, evaluated, and replaced by functional alternative cognitions (e.g. "When I finish my work, I will reward myself by taking a walk in the park").

So far, trials testing CBT for obesity generally show statistically significant but modest weight loss and long-term weight loss maintenance results (Sbrocco, Nedegaard, Stone, \& Lewis, 1999; Stahre \& Hällström, 2005; Stahre, Tärnell, Håkanson, \& Hällström, 2007; Werrij et al., 2009, but see Cooper et al., 2010). However, the influence of cognitions and emotions on eating behavior is still poorly understood: most previous research relied on questionnaires to assess cognitions (Nauta, Hospers, Kok, \& Jansen, 2000; O'Connor \& Dowrick, 1987), or manipulated emotions in the lab (see Cardi, Leppanen, \& Treasure, 2015 for an overview). So, more research is needed to further elucidate their role from the perspective of daily life. Obesity treatment may also benefit from being provided indefinitely, as it is sometimes considered a chronic condition (Bray, 2004; Perri, 1998; Rippe et al., 1998), and from treatment directly when food desires are experienced, or when dietary temptation occurs. To deal with these considerations, CBT for obesity treatment could benefit from a relatively low-cost, automated form of intervention that has the capacity to intervene in daily life, called ecological momentary intervention (EMI).

Chapter $\mathbf{2}$ contains an investigation of eating-related cognitions and emotions in daily life in overweight and healthy-weight participants. Three hypotheses were tested: (1) The CBT model for obesity suggests that dysfunctional cognitions are important antecedents 
of high-caloric food intake, and should therefore occur more often prior to high-caloric food intake for overweight than for healthy-weight participants. (2) For emotions, previous studies have found that negative emotions play a role in promoting overweight individuals high-caloric food intake (Cardi et al., 2015), and so negative emotions should also occur more often specifically when about to eat high-caloric food for overweight than for healthyweight participants. (3) A secondary aim was to investigate an eating style called 'emotional eating' (Arnow, Kenardy, \& Agras, 1995; van Strien et al., 1986). Scores on emotional eating questionnaires may reflect beliefs about eating behavior, rather than the actual eating behavior (Adriaanse et al., 2011; Bongers et al., 2016; Evers et al., 2009). It was therefore hypothesized that such scores would be positively associated with the occurrence of negative emotions prior to any assessment type: high-caloric eating events, non-high-caloric eating events, and non-eating moments.

To investigate these hypotheses, an iPhone app was used that prompted participants to complete brief questionnaires (assessments / reports) throughout the day. In addition, participants were instructed to complete a questionnaire immediately prior to eating. For the present thesis, the questions assessing eating-related cognitions, emotions, food desires and food intake were included in the analyses. Results showed that, contrary to expectations based on the CBT model of obesity, dysfunctional cognitions only occurred very infrequently, and did not occur more often for overweight relative to healthy-weight participants. So, the most likely conclusion is that dysfunctional cognitions right before eating do not play a major role in obesity and overeating.

For emotions, the main findings were that overweight participants were more emotionally negative than healthy-weight participants, not only when about to eat, but also randomly throughout the day. So, again contrary to expectations from the CBT model and previous research, negative emotions were not more strongly associated specifically with high-caloric eating events for overweight than for healthy-weight participants. Similarly, for participants who scored high on emotional eating questionnaires, negative emotions did not occur more often specifically prior to high-caloric eating events than those who scored low. Concluding, these findings imply that eating-related thoughts and emotions, as assessed by EMA, do not differentiate overweight and healthy-weight individuals well.

Chapter $\mathbf{3}$ is based on the same EMA study as Chapter 2, but presents the data on food desire and food intake. Previous research showed that overweight participants may be more susceptible to the rewarding value of food, leading to more frequent food desires. In addition, overweight participants were previously found to consume more varied highcaloric foods. In Chapter 3, the hypothesis was tested that overweight participants have more frequent (high-caloric) food desires, more food intake, and more varied (high-caloric) food desires and food intake than healthy-weight participants. Results showed that, indeed, overweight participants reported more high-caloric food desires than healthy-weight participants, specifically at non-eating moments. Healthy-weight participants, on the other 
hand, reported more desires for staple foods (food products that are eaten routinely, such as rice and bread), than overweight participants, specifically prior to eating events. It was also found that high-caloric food desires of overweight participants were more varied than those of healthy-weight participants, both at eating events and at non-eating moments. However, intake variety did not differ between participant groups. This discrepancy might occur because food intake was only accompanied by a specific food desire in around $40 \%$ of the eating events, making it less likely to find differences between participant groups for food intake. In sum, it may be important for obesity interventions to specifically target high-caloric food desires that are experienced when not eating, and to aim for a reduction in the variety of high-caloric food desire.

Chapter 4 presents the design and protocol of an RCT comparing a CBT-based EMI for weight loss (Think Slim) with a self-guided diet-only control condition. The RCT lasted six weeks, and was preceded and followed by a measurement week, during which participants obtained a measurement of BMI, completed a battery of questionnaires (a questionnaire assessing belief in dysfunctional cognitions, the Dutch Eating Behavior Questionnaire, the Eating Disorder Examination Questionnaire, the Self-Liking and Self-Competence scale, and the Brief Symptom Inventory), and performed EMA (with no intervention taking place). There were also two follow-ups of the outcome measures (at 3 months and 12 months post-intervention).

Participants in the Think Slim condition used an iPhone app, which provided treatment promptly, and were provided CBT sessions in a web-based environment, with no therapist involvement. The CBT sessions were themed around teaching participants how to distinguish food desires from hunger, how to formulate concrete weight loss goals, how to identify and evaluate dysfunctional cognitions, how to increase weight- and shape-related self-esteem, and how to prevent weight regain.

The app was intended as a practical tool for self-monitoring (via EMA) and consisted of three CBT-related treatment modules. (1) The passive feedback module compiled graphs and charts of the EMA data, which showed how food desire was related to emotions, and how high-caloric and non-high-caloric food intake were related to activities and locations. (2) The adaptive feedback module was a self-learning algorithm (Spanakis et al., 2016) that estimated, using previously entered EMA data, when participants were likely to report high-caloric eating events. At non-eating assessments, participants received a warning and CBT-related instruction whenever the app detected it was likely that(s)he would overeat in a subsequent time period (of, on average, two hours). (3) Using the thought evaluation module, participants could evaluate dysfunctional cognitions using self-guiding questions, and formulate functional alternative cognitions, and could review their dietary goals and evaluated cognitions.

Chapter 5 contains the results of the RCT comparing Think Slim with a self-guided dietonly control. For BMI, questionnaires assessing belief in dysfunctional cognitions, emotional 
/ external / restrained eating, eating disorder symptomatology, general psychopathology, and self-esteem, and EMA data on cognitions and emotions, it was hypothesized that Think Slim would outperform the self-guided diet-only control, and therefore lead to more weight loss, and more improvements on the other outcome measures. Additionally, dietary adherence was investigated, and it was hypothesized that diet adherence would be positively associated with weight loss and improvements on self-assessed dysfunctional cognitions, emotional eating, external eating, and restrained eating.

Contrary to expectations, Think Slim did not result in more weight loss than the diet-only control condition. Weight loss in both conditions was statistically significant at post measurement, but modest. In line with the hypotheses, only participants in the Think Slim condition reported less belief in dysfunctional cognitions, less emotional and external eating, and more restrained eating, when comparing post-intervention scores with baseline. These results were partly corroborated by the EMA data. Participants of the Think Slim condition, but not of the self-guided diet-only control, reported fewer dysfunctional cognitions, when comparing post-intervention with baseline. However, contrary to expectations, participants in the Think Slim condition did not report less negative emotions prior to high-caloric eating post-intervention, compared to baseline. Furthermore, both conditions reduced significantly in reported eating disorder symptomatology. The modest weight loss results of the present RCT were comparable to previous studies investigating CBT for weight loss (Sbrocco et al., 1999; Stahre \& Hällström, 2005; Stahre et al., 2007; Werrij et al., 2009), which resulted in long-term weight loss maintenance. So, this is an indication that the follow-up results of the present RCT could also yield weight loss maintenance for the Think Slim, but not for the self-guided diet-only control condition.

Chapter 6 presents a discussion of the studies and outcomes of the present thesis. Main conclusions include: (1) Contrary to what the CBT model suggests, there was no evidence for a strong role of eating-related dysfunctional cognitions and (negative) emotions in the eating behavior of overweight individuals. (2) Relatedly, emotional eating did not specifically reflect a strong association between negative emotions and high-caloric eating, so it is suggested to instead reflect a belief about this association. (3) High-caloric dietary temptations should be targeted by obesity interventions specifically when overweight participants are not eating, and obesity interventions should focus on reducing variety of high-caloric food desires. (4) Using a CBT-based EMI for weight loss did not result in more weight loss than a diet-only control, but did result in a reduction in belief in dysfunctional cognitions, emotional eating and external eating, which was not achieved by the diet-only control. These results are hopeful for weight maintenance of the EMI condition.

Lastly, several suggestions for future research are made. (1) Rather than target eatingrelated dysfunctional cognitions immediately prior to eating events, future research could focus on eating-related cognitions that occur at an earlier moment (e.g., in a supermarket). 
(2) It is also useful to investigate methods for maintaining a high EMA compliance. (3) Lastly, future EMA / EMI research could be improved by incorporating GPS sensor technology. 

Nederlandse Samenvatting 

Het doel van dit proefschrift was om de effectiviteit van CBT voor obesitas in de context van het dagelijks leven te onderzoeken. De CBT voor obesitas werd gegeven als EMI, via een iPhone applicatie en een online platform. Als eerste was de rol van cognities, emoties, trek en voedselinname onderzocht in het dagelijks leven, via EMA, en vergeleken tussen deelnemers met en zonder overgewicht. Als tweede was de EMI voor obesitas ('Think Slim') vergeleken met een alleen-dieet controle groep. De hoofdbevindingen zullen hier worden samengevat.

Hoofdstuk 1 bevat een overzicht van het doel van dit proefschrift vanuit een theoretisch oogpunt. Het merendeel mensen met overgewicht is regelmatig aan het diëten, en er zijn veel interventies voor gewichtsverlies. Het succes van diëten en gewichtsverlies is echter in algemene zin laag, vooral op lange termijn (e.g. Franz et al., 2007; Mann et al., 2007). De meeste mensen bereiken niet het gewichtsverlies waarop ze gehoopt hadden, en komen uiteindelijk weer aan (Cooper et al., 2010). Het model van cognitieve gedragstherapie (CBT) voor obesitas zegt dat diëten en gewichtsverlies op de lange termijn effectiever kunnen zijn door dysfunctionele gedachten (cognities) aan te pakken, die ervoor kunnen zorgen dat mensen teveel blijven eten ondanks de negatieve lange termijn consequenties van gewichtstoename (Carter \& Jansen, 2012; Jansen \& Mulkens, 2013; Jansen, Nederkoorn, Roefs, Martijn, \& Havermans, 2009; Karhunen et al., 2012). Zulke dysfunctionele cognities (e.g., "Als ik klaar ben met werken, kan ik mezelf belonen met een snack") houden een vicieuze cirkel van inadequate pogingen tot gewichtsverlies in stand, doordat negatieve emoties worden versterkt, zelfwerkzaamheid en zelfvertrouwen worden verlaagd, en zorgen over eetgedrag, lichaamsvormen en gewicht worden versterkt. Dit leidt tot hoogcalorische eetmomenten. Om deze vicieuze cirkel te verbreken, dienen dysfunctionele cognities te worden geïdentificeerd, geëvalueerd en vervangen door functionele alternatieve cognities (e.g. "Als ik klaar ben met mijn werk, beloon ik mezelf door in het park te gaan wandelen").

Tot nu toe tonen behandelstudies aan dat CBT voor obesitas over het algemeen leidt tot statistisch significant, maar middelmatig gewichtsverlies en behoud van gewichtsverlies op de lange termijn (Sbrocco, Nedegaard, Stone, \& Lewis, 1999; Stahre \& Hällström, 2005; Stahre, Tärnell, Håkanson, \& Hällström, 2007; Werrij et al., 2009, maar zie Cooper et al., 2010). Echter, de invloed van cognities en emoties op eetgedrag is nog steeds slecht begrepen: het meeste voorafgaande onderzoek gebruikte vragenlijsten om cognities te meten (Nauta, Hospers, Kok, \& Jansen, 2000; O'Connor \& Dowrick, 1987), of manipuleerde emoties in het lab (zie Cardi, Leppanen, \&Treasure, 2015 voor een overzicht). Dus, meer onderzoek is nodig om de rol van cognities te benadrukken vanuit het perspectief van het dagelijks leven. Obesitas behandelingen kunnen daarnaast verbeterd worden door de behandeling onbeperkt (zonder einde) te geven, aangezien obesitas soms als chronische aandoening wordt gezien (Bray, 2004; Perri, 1998; Rippe et al., 1998), en van behandelingen die plaatsvinden zo gauw er trek in voedsel optreedt. Om aan deze suggesties te voldoen, zou CBT voor obesitas 
kunnen worden verbeterd via een relatief kostenefficiënte manier van interveniëren in het dagelijks leven, genaamt ecologische momentane interventie (EMI).

Hoofdstuk 2 bevat een onderzoek naar eet-gerelateerde cognities en emoties in het dagelijks leven, van deelnemers met en zonder overgewicht. Drie hypothesen werden onderzocht: (1) Het CBT model voor obesitas suggereert dat dysfunctionele cognities belangrijke antecedenten zijn van hoog-calorische voedselinname. Dus, zulke cognities zouden vaker moeten voorkomen vlak voor inname van hoog-calorisch voedsel door deelnemers met overgewicht, in vergelijking met deelnemers zonder overgewicht. (2) Voor emoties lieten vorige studies zien dat negatieve emoties een rol speelden in het bevorderen van hoogcalorische voedselinname van mensen met overgewicht (Cardi et al., 2015). Daarom zouden negatieve emoties vaker moeten voorkomen specifiek wanneer er hoog-calorisch gegeten gaat worden door deelnemers met overgewicht dan door deelnemers zonder overgewicht. (3) Een secundair doel was het onderzoeken van een eetstijl genaamd 'emotioneel eten' (Arnow, Kenardy, \& Agras, 1995; van Strien et al., 1986). Het is gesuggereerd dat scores op vragenlijsten die emotioneel eten meten meer zeggen over het geloof dat mensen hebbben omtrent dingen die samenhangen met hun eetgedrag, in plaats van over daadwerkelijk eetgedrag (Adriaanse et al., 2011; Bongers et al., 2016; Evers et al., 2009). De hypothese hierbij was daarom dat zulke vragenlijstscores positief geassocieerd zijn met het voorkomen van negatieve emoties vlak voor wat voor meetmoment dan ook: hoog-calorische eetmomenten, niet-hoog-calorische eetmomenten, en niet-eetmomenten.

Om deze hypothesen te onderzoeken was er een iPhone app ontwikkeld en gebruikt, die deelnemers in staat stelde korte vragenlijsten (rapportages) in te vullen gedurende de dag. Daarnaast waren deelnemers geïnstrueerd om de rapportages in te vullen vlak voordat ze iets gingen eten. De huidige these richt zich hierbij op de vragen over eet-gerelateerde cognities, emoties, trek, en voedselinname. Resultaten laten zien dat, in tegenstelling tot de verwachtingen vanuit het CBT model voor obesitas, dysfunctionele cognities slechts sporadisch voorkwamen, en niet vaker voor mensen met overgewicht dan voor mensen zonder overgewicht. Dus, de meest waarschijnlijke conclusie is dat dysfunctionele cognities vlak voor een eetmoment geen grote rol spelen in obesitas en overeten.

Wat betreft emoties lieten de bevindingen zien dat deelnemers met overgewicht meer emotioneel negatief waren dan deelnemers zonder overgewicht; niet alleen wanneer ze op het punt stonden iets te eten, maar ook willekeurig gedurende de dag. Dus, wederom in tegenstelling tot de verwachtingen vanuit eht CBT model en eerder onderzoek, negatieve emoties waren niet sterker geassocieerd met hoog-calorische voedselinname bij mensen met overgewicht dan bij mensen zonder overgewicht. Daarnaast was het zo, dat deelnemers die hoog scoorden op emotioneel eten vragenlijsten niet vaker negatieve emoties rapporteerden vlak voor hoog-calorische eetmomenten dan deelnemers die laag scoorden. Dus, deze bevindingen laten zien dat eet-gerelateerde cognities en emoties, zoals gemeten 
door EMA, niet goed differentiëren tussen mensen met overgewicht en mensen zonder overgewicht.

Hoofdstuk $\mathbf{3}$ is gebaseerd op hetzelfde EMA onderzoek als Hoofdstuk 2, maar betreft data over trek en voedselinname. Voorgaand onderzoek liet zien dat mensen met overgewicht vatbaarder zijn voor de belonende waarde van voedsel, waardoor ze vaker trek hebben. Daarnaast werd aangetoond dat mensen met overgewicht gevarieerder hoog-calorisch voedsel tot zich nemen dan deelnemers zonder overgewicht. De hypothese die in Hoofdstuk 3 was getest, stelde dat deelnemers met overgewicht vaker (hoog-calorische) trek hebben, meer voedselinname hebben, en gevarieerdere trek en voedselinname hebben dan deelnemers zonder overgewicht. Resultaten lieten zien dat deelnemers met overgewicht inderdaad meer trek in hoog-calorisch voedsel hadden dan deelnemers zonder overgewicht, specifiek wanneer ze niet op het punt stonden iets te eten. Daarnaast rapporteerden deelnemers zonder overgewicht vaker trek in voedsel dat vaak gegeten wordt bij het ontbijt, de lunch, of het avondeten (zoals rijst en brood), dan deelnemers met overgewicht, specifiek wanneer ze op het punt stonden iets te eten. Ook hadden deelnemers met overgewicht gevarieerdere hoog-calorische trek dan deelnemers zonder overgewicht, zowel op eetmomenten als op niet-eetmomenten. Echter, variatie in voedselinname verschilde niet tussen de groepen. Deze discrepantie zou kunnen zijn ontstaan doordat voedselinname slechts in ongeveer 40\% van de gevallen samen ging met specifieke trek, waardoor het vinden van verschillen tussen de groepen minder waarschijnlijk was. In het kort, het is wellicht belangrijk voor obesitas interventies om specifiek te richten op hoog-calorische trek wanneer er niet gegeten wordt, en om een reductie tot stand te brengen in de variatie in hoog-calorische trek.

Hoofdstuk 4 vertegenwoordigt het ontwerp en protocol van een RCT waarin een op CBT gebaseerde EMI voor gewichtsverlies (Think Slim) wordt vergeleken met een alleen-dieet groep. De RCT duurde zes weken en was vooraf gegaan en gevolgd door een meetweek, waarin deelnemers zich lieten wegen en meten, en een aantal vragenlijsten invulden (een vragenlijst die geloof in dysfunctionele cognities meet, de Nederlandse Vragenlijst voor Eetgedrag, de Eating Disorder Examination Questionnaire, de Self-Liking and Self-Competence schaal, en de Brief Symptom Inventory), en aan EMA deden (zonder dat er interventie plaatsvond). Er waren daarnaast twee vervolgmetingen (op 3 maanden en 12 maanden na de interventie).

Deelnemers in de Think Slim groep gebruikten een iPhone applicatie die behandelde op het moment dat het nodig was, en volgden daarnaast CBT sessies in een webomgeving, zonder dat daarbij een therapeut betrokken was. De CBT sessies richtten zich op het aanleren van deelnemers om trek van honger te onderscheiden, het formuleren van concrete doelen voor gewichtsverlies, het identificeren en evalueren van dysfunctionele cognities, het vergroten van zelfvertrouwen omtrent gewicht en vorm, en het voorkomen van gewichtstoename na het afvallen. 
De app was bedoeld als praktisch handvat om zelf-monitoring te bevorderen (via EMA) en bestond uit drie CBT-gerelateerde modules. (1) De passieve feedback module compileerde grafische overzichten en tabellen van de EMA data, waardoor deelnemers inzicht kregen in de relatie tussen trek en emoties, en hoe hoog-calorische en niet-hoog-calorische voedselinname gerelateerd waren met activiteiten en locaties. (2) De adaptieve feedback module bestond uit een zelf-lerend algoritme (Spanakis et al., 2016) dat inschatte, via vooraf ingevoerde EMA data, wanneer deelnemers waarschijnlijk hoog-calorische eetmomenten zouden rapporteren. Op niet-eetmomenten ontvingen deelnemers waarschuwingen en CBT-gerelateerde adviezen wanneer de app detecteerde dat het waarschijnlijk was dat ze zouden gaan overeten in de daaropvolgende tijdperiode (van gemiddeld twee uur). (3) Via de gedachten evaluatie module konden deelnemers dysfunctionele cognities evalueren middels open vragen, en functionele alternatieve cognities formuleren, en hun dieet doelen en geëvalueerde cognities opnieuw oproepen en bekijken.

Hoofdstuk 5 bevat de resultaten van de RCT waarin de Think Slim interventie werd vergeleken met een alleen-dieet groep. De belangrijkste hypothese bij deze vergelijking, was dat Think Slim het beter zou doen dan de alleen-dieet groep, als het gaat om gewichtsverlies, vermindering van geloof in dysfunctionele cognities, emotioneel / extern / lijngericht eten, eetstoornis psychopathologie, algemene psychopathologie en een verbetering in zelfvertrouwen. Daarnaast was aanhankelijkheid tot het volgen van het dieet onderzocht. Hierbij was de hypothese dat dieet-aanhankelijkheid positief geassocieerd is met gewichtsverlies en verbeteringen in geloof in dysfunctionele cognities, emotioneel eten, extern eten en lijngericht eten.

In tegenstelling tot de verwachtingen leidde Think Slim niet in meer gewichtsverlies dan de alleen-dieet groep. Gewichtsverlies in beide groeps was statistisch significant op de nameting, maar middelmatig. Wel in lijn met de hypotheses was gevonden dat alleen deelnemers in de Think Slim groep op de nameting (in vergelijking met de voormeting) minder geloof in dysfunctionele cognities rapporteerden, minder emotioneel en extern eten, en meer lijngericht eten, dan de alleen-dieet groep. Deze resultaten waren deels ondersteund door de EMA data. Deelnemers van de Think Slim groep, maar niet die van de alleen-dieet groep, rapporteerden minder dysfunctionele cognities na de interventie dan vooraf. Echter, in tegenstelling tot de verwachtingen, rapporteerden deelnemers in de Think Slim groep niet minder negatieve emoties vooraf aan hoog-calorische eetmomenten na de interventie in vergelijking met vooraf, dan de deelnemers in de alleen-dieet groep. Daarnaast reduceerden beide groepen significant in eetstoornis symptomatologie. Het middelmatige gewichtsverlies van deze huidige RCT is vergelijkbaar met voorgaand onderzoek naar CBT voor gewichtsverlies (Sbrocco et al., 1999; Stahre \& Hällström, 2005; Stahre et al., 2007; Werrij et al., 2009), waarin het lange-termijn behoud van het verloren gewicht ook werd gevonden. Dit is een indicatie dat de lange-termijn resultaten van de huidige RCT ook zouden kunnen leiden tot gewichtsbehoud voor Think Slim, maar niet voor de alleen-dieet groep. 
Hoofdstuk 6 is een discussie van de studies en resultaten van de huidige these. De hoofdconclusies luiden: (1) In tegenstelling tot wat het CBT model voor obesitas suggereert, was er geen bewijs voor een sterke rol van eet-gerelateerde dysfunctionele cognities en (negatieve) emoties als verklaring voor het eetgedrag van mensen met overgewicht. (2) Hieraan gerelateerd, emotioneel eten reflecteerde niet specifiek een sterke associatie tussen negatieve emoties en hoog-calorische eetmomenten. Daarom kan worden gesuggereerd dat emotioneel eten in plaats daarvan gaat over het geloof dat mensen hebben in deze associatie tussen negatieve emoties en eetgedrag. (3) Hoog-calorische trek zou een pijler moeten vormen van obesitas interventies, juist wanneer mensen met overgewicht niet aan het eten zijn, en interventies voor obesitas zouden zich moeten richten op het reduceren van de variatie in hoog-calorische trek. (4) Een op CBT gebaseerde EMI voor gewichtsverlies leidde niet tot meer gewichtsverlies dan het volgen van een dieet, maar leidde wel tot een reductie in geloof in dysfunctionele cognities, emotioneel eten, en extern eten, terwijl de groep die alleen een dieet volgde deze reducties niet bereikte. Deze resultaten zijn daarom hoopvol als het gaat om het succesvol behouden van het (nieuwe) gewicht voor deelnemers in de EMI groep.

Als laatste worden er enkele suggesties voor toekomstig onderzoek gedaan. (1) In plaats van het richten op eet-gerelateerde cognities vlak voor eetmomenten, zou toekomstig onderzoek zich kunnen richten op eet-gerelateerde cognities die zich voordoen op eerdere momenten (e.g., in een supermarkt). (2) Het is ook nuttig om methoden te onderzoeken die een hoge navolging van EMA bevorderen. (3) Als laatste zou toekomstig EMA onderzoek kunnen worden verbeterd door het toevoegen van GPS sensor technologie. 



\section{Valorisation}





\section{RELEVANCE}

Obesity (Body Mass Index; BMI > 25kg/m²) is considered a pandemic (Swinburn et al., 2011), as its worldwide prevalence is now at over $37 \%$ of the population ( $\mathrm{Ng}$ et al., 2014). This is problematic, as obesity has a negative impact on physiological and psychological health. In addition, obesity is associated with social stigma (Puhl \& Heuer, 2010). Obesity-related conditions, such as type-2 diabetes and cardiovascular disease (Dixon, 2010) are a significant healthcare burden. The US obesity-related annual medical healthcare costs is about $20.6 \%$, at 209.7 billion (Cawley \& Meyerhoefer, 2012).

These detrimental effects make obesity an undesired state, and as a result many obese people resort to dieting. A Dutch study found that more people (around 63\%) are currently dieting than there are obese people in the Dutch population (De Ridder, Adriaanse, Evers, \& Verhoeven, 2014), which implies that many people are concerned about what they eat. However, the majority of dieting attempts are unsuccessful in the long run, and weight loss interventions in general do not result in effective, sustained weight loss (e.g., Franz et al., 2007). In fact, obesity is sometimes considered a chronic condition (Bray, 2004; Rippe, Crossley, \& Ringer, 1998). It is, however, clear that, given the detrimental effects of obesity on health and the economy, research on effective obesity interventions and antecedents and consequences of (over)eating behavior should continue (Brownell, 2010).

The studies conducted in the present thesis primarily contribute to the fields of eating behavior research and research on effectiveness of obesity interventions. The first goal of the present thesis was to investigate cognitive behavior therapy (CBT)-related aspects of eating behavior in people's daily lives. CBT is a prominent treatment with application to many different psychological disorders (e.g., Beck, 2005). For obesity, so far, some trials have shown that CBT might lead to moderate long term weight loss maintenance (Stahre \& Hällström, 2005; Stahre, Tärnell, Håkanson, \& Hällström, 2007; Werrij et al., 2009).

A central component of CBT is the identification and evaluation of dysfunctional cognitions, and the influence these cognitions have on the occurrence of (negative) emotions (Beck, 2011). However, not much is known about the kinds of eating-related cognitions people have, and about whether cognitions of overweight and healthy-weight people differ. The results presented in Chapter 2 illustrated that cognitions and emotions right before eating may not be effective targets for obesity interventions, as most cognitions were not dysfunctional, and instead were about food desire and taste. In addition, there were hardly any differences between overweight and healthy-weight participants, and negative emotions occurred just as often prior to eating events than to non-eating moments.

The second, and central goal of the thesis, was to investigate possibilities for improving obesity treatment, by designing and testing a CBT-based weight loss treatment (Think Slim) that relied entirely on mobile technology and the internet, provided in addition to a regular calorie restrictive diet, with no therapist involvement. Proving treatment this way has sev- 
eral advantages that are of general relevance. First, it is possible to provide (psychological) obesity treatment without active, in-person consultation with an expert throughout the treatment, which is relatively cost-efficient (Agras, Taylor, Feldman, Losch, \& Burnett, 1990). This treatment can be tailored to individually relevant moments (e.g., moments of dietary weakness). Second, the barrier to starting with treatment is lowered, as there is no longer a need to physically visit clinics. Lastly, another economic advantage is that treatment provision can be indefinite, so there is no longer a need to prepare a client to apply the learned therapeutic techniques independently. This is especially relevant for obesity, as it is sometimes considered a chronic condition (Bray, 2004; Fujioka, 2002). The app, as a therapeutic tool, will always be available, and treatment sessions can be reviewed at any time.

The Think Slim treatment was more effective at reducing belief in dysfunctional cognitions, and emotional / external eating than a diet-only control condition. However, both conditions lost similar (modest) weight. So, there is still need for future research on CBT for obesity and / or on treatment of obesity in daily life before a general recommendation can be provided. Such future research should focus on disentangling what aspects of treatment could be improved. For example, the duration of the Think Slim treatment was relatively short ( 6 weeks). It is possible that differences between the Think Slim treatment and the diet-only control only become apparent after a longer treatment period. Also, as compliance with the Think Slim treatment reduced greatly over time, it is essential that methods for avoiding this reduction in compliance are investigated. For CBT itself, it is important to investigate cognitions not only when about to eat, but also at different relevant moments (e.g., in the supermarket). Most importantly, however, as the emphasis for effective weight loss treatment is on the follow-up period results (i.e., weight loss maintenance), it is still possible that participants in the Think Slim treatment will be able to maintain their weight loss, whereas participants in the control condition will regain the lost weight. So, it is important to wait until the follow-up results of the Think Slim trial are available, before any firm conclusions can be drawn.

\section{RELEVANCE TO SPECIFIC TARGET GROUPS}

Aside of general relevance, the findings of the present thesis are relevant to three target groups. (1) Overweight population: Both studies in the present thesis included relatively large and heterogeneous samples of overweight (and, for the first study, healthy-weight) participants. Included participants varied widely in terms of age, socioeconomic status, education level, and BMI. So, the presented findings can be generalized to the entire (Dutch) overweight/obese population. (2) Healthcare professionals: Although there was no therapist contact in either of the presented studies, investigating eating behavior in daily life is relevant to clinical settings. By mixing therapist contact with reliance on smartphone 
and/or internet tools, face-to-face therapy can be enhanced. This so-called blended therapy (e.g., van der Vaart et al., 2014), has the advantages of being able to obtain insights into clients' eating behavior promptly in daily life (e.g., by having clients conduct EMA). However, therapeutic sessions and intervention can be provided in a face-to-face manner. This also provides new opportunities for obesity intervention research, as not much is known about blended therapy for obesity that makes use of EMA. (3) Health insurance companies: A previous review of obesity interventions concluded that including obesity treatment in the standard healthcare package could not be recommended, as the effectiveness of such treatment was not established (Mann et al., 2007). Since then, there has not been much improvement in terms of effectiveness (Brownell, 2010) So, in this case, the cost of treatment would not outweigh the outcome benefits on health. However, by conducting treatment via a smartphone app and the internet, treatment costs are reduced (once a treatment platform is developed), which makes this form of treatment provision especially interesting as an alternative to traditional face-to-face therapy.

\section{TRANSLATION INTO INNOVATIVE ACTIVITIES AND PRODUCTS}

There are two distinct directions of development that are relevant in this respect. First, in the RCT presented in Chapter 5, computational algorithms were used to construct 'eating profiles' for each participant (See: Spanakis, Boh, Weiss \& Roefs, 2016). Such profiles relied on knowledge of participants' previous (over)eating behavior to estimate when they were at risk for new (over)eating events. So, the app continuously tailored to individually relevant moments. Briefly, to be able to predict risk factors for overeating, a novel computational algorithm was developed, which relied on decision tree modeling to cluster overweight participants into separate groups, based on how similar they were in terms of antecedents of overeating behavior. Treatment was then provided in the form of a warning and CBTbased feedback message. This algorithm can be applied to future research that uses EMA data to estimate what combinations of states of variables may lead to undesirable behavior, and can help prevent overeating altogether.

Secondly, the Think Slim app could be expanded by incorporating sensor technology. Increasingly often, different sorts of sensors are included in mobile devices. Using such sensors has the benefit of reducing the self-report aspect of EMA (relying on people to make judgments about their own behavior, thoughts, and emotions), and instead using objective measures of physiological states and geographical locations. In addition, people will spend less time completing the EMA questionnaire (as less self-report questions will need to be included). Sensors that are useful for eating behavior could be related to external factors (e.g., GPS or accelerometry), or internal states (e.g., by measuring heart rate). Some of these sensor technologies are more thoroughly investigated than others. In general, it is essential 
that the predictive quality of a sensor is not impeded by noisy measurements (e.g., making sure that a certain signal is relevant to an overeating event). For example, although automatic (unobtrusive) facial recognition of emotions might be interesting, as it is a potentially objective measure of emotions, it is currently not reliable enough to be implemented in clinical settings, with one study reporting accurate recognition in 55 to $76 \%$ of cases (Asselbergs, Ruwaard, Ejdys, Schrader, Sijbrandij, \& Riper, 2016). However, on the other hand, GPS and accelerometry are already very accurate, and fairly easy to implement, as most mobile devices can track physical locations (e.g., using Google Maps) and can count steps / physical exertion. GPS and accelerometry have already been successfully applied with high accuracy in several devices and mobile systems (e.g., Doherty, Lemieux \& Canally, 2014; Gaggioli et al., 2011).

A separate development that resulted from the work conducted in the present thesis is fostering and enhancing inter-faculty and inter-university knowledge exchange and cooperation, and increasing contact between universities and companies. To develop the Think Slim app, extensive collaboration occurred between the Maastricht University Faculty of Psychology and Neuroscience, and the Faculty of Humanities and Sciences (specifically the Department of Data Science \& Knowledge Engineering). This has resulted in open and future-oriented scientific collaborations.

\section{IMPLEMENTATION}

The Maastricht University Instrumentation department is currently in the process of expanding the functionality of the Think Slim app to suit more general research needs. This update of the Think Slim app will allow researchers to script their own research designs (e.g., what sorts of EMA questions to ask, and how to score the questions). A major advantage of having such an app available within the university is that future EMA research projects do not have to rely on, and invest in, commercially available alternatives. Also, support staff is available immediately to fix any technical issues that may arise during development and during a study. One potential risk that can be identified in relation to EMA app development is that smartphone usage might decline when different forms of technology become popular. 
Reference list 

Aardoom, J. J., Dingemans, A. E., Slof Op't Landt, M. C. T., \& Van Furth, E. F. (2012). Norms and discriminative validity of the Eating Disorder Examination Questionnaire (EDE-Q). Eating Behaviors, 13, 305-309.

Abramson, E. E., \& Stinson, S. G. (1977). Boredom and eating in obese and non-obese individuals. Addictive Behaviors, 2, 181-185.

Adriaanse, M. A., de Ridder, D. T. D., \& Evers, C. (2011). Emotional eating: eating when emotional or emotional about eating? Psychology \& Health, 26, 23-39.

Agras, W. S., Taylor, C. B., Feldman, D. E., Losch, M., \& Burnett, K. F. (1990). Developing computer-assisted therapy for the treatment of obesity. Behavior Therapy, 21, 99-109.

van den Akker, K., Havermans, R. C., \& Jansen, A. (2015). Effects of occasional reinforced trials during extinction on the reacquisition of conditioned responses to food cues. Journal of Behavior Therapy and Experimental Psychiatry, 48, 50-58.

van den Akker, K., Jansen, A., Frentz, F., \& Havermans, R. C. (2013). Impulsivity makes more susceptible to overeating after contextual appetitive conditioning. Appetite, 70, 73-80.

Amir, N., \& Taylor, C. T. (2012). Combining computerized home-based treatments for generalized anxiety disorder: an attention modification program and cognitive behavioral therapy. Behavior Therapy, 43 , 546-59.

Anderson, J. W., Konz, E. C., Frederich, R. C., \& Wood, C. L. (2001). Long-term weight-loss maintenance: a metaanalysis of US studies. American Journal of Clinical Nutrition, 74, 579-84.

Andreu, Y., Galdón, M. J., Dura, E., Ferrando, M., Murgui, S., García, A., \& Ibáñez, E. (2008). Psychometric properties of the brief symptoms inventory-18 (BSI-18) in a Spanish sample of outpatients with psychiatric disorders. Psicothema, 20, 844-850.

Andrews, G., Cuijpers, P., Craske, M. G., McEvoy, P., \& Titov, N. (2010). Computer therapy for the anxiety and depressive disorders is effective, acceptable and practical health care: a meta-analysis. Plos One, 5, e13196.

Arnow, B., Kenardy, J., \& Agras, W. S. (1995). The emotional eating scale: The development of a measure to assess coping with negative affect by eating. International Journal of Eating Disorders, 18, 79-90.

Ashton, K., Drerup, M., Windover, A., \& Heinberg, L. (2009). Brief, four-session group CBT reduces binge eating behaviors among bariatric surgery candidates. Surgery for Obesity and Related Diseases, 5, 257-262.

Atallah, R., Filion, K. B., Wakil, S. M., Genest, J., Joseph, L., Poirier, P., ... Eisenberg, M. J. (2014). Long-term effects of 4 popular diets on weight loss and cardiovascular risk factors: A systematic review of randomized controlled trials. Circulation: Cardiovascular Quality and Outcomes, 7, 815-827.

Atienza, A. A., King, A. C., Oliveira, B. M., Ahn, D. K., \& Gardner, C. D. (2008). Using hand-held computer technologies to improve dietary intake. American Journal of Preventive Medicine, 34, 514-8.

Atkinson, N. L., Gold, R. S., \& Ballard, G. (2002). The Promise and Challenge of eHealth Interventions. American Journal of Health Behavior, 26, 494-503.

Beck, J. S. (2007). The Beck Diet Solution: Train your Brain to Think Like a Thin Person. Birmingham, Alabama: Oxmoor House, Inc.

Beck, J. S. (2008). Beck's dieetoplossing: Train je brein, denk slank. (M. van der Horst, Trans.). Amsterdam: Uitgeverij Nieuwezijds. (Original work published in 2007).

Beck, J. S. (2011). Cognitive Behavior Therapy: Basics and beyond. London, NY: Guildford Press.

Bennett, G. G., \& Glasgow, R. E. (2009). The delivery of public health interventions via the Internet: actualizing their potential. Annual Review of Public Health, 30, 273-92.

Berg, K. C., Peterson, C. B., Frazier, P., \& Crow, S. J. (2012). Psychometric evaluation of the eating disorder examination and eating disorder examination-questionnaire: A systematic review of the literature. International Journal of Eating Disorders, 45, 428-438. 
Berkman, E. T., Giuliani, N. R., \& Pruitt, A. K. (2014). Comparison of text messaging and paper-and-pencil for ecological momentary assessment of food craving and intake. Appetite, 81, 131-7.

de Beurs, E. (2006). Brief Symptom Inventory: Handleiding. Leiden: PITS.

Blakemore, A. I. F., \& Froguel, P. (2008). Is obesity our genetic legacy? Journal of Clinical Endocrinology and Metabolism, 93, S51-6.

Blundell, J. E., Stubbs, R. J., Golding, C., Croden, F., Alam, R., Whybrow, S., ... Lawton, C. L. (2005). Resistance and susceptibility to weight gain: individual variability in response to a high-fat diet. Physiology \& Behavior, $86,614-22$.

Boh, B., Lemmens, L. H. J. M., Jansen, A., Nederkoorn, C., Kerkhofs, V., Spanakis, G., .. Roefs, A. (2016). An Ecological Momentary Intervention for weight loss and healthy eating via smartphone and Internet: study protocol for a randomised controlled trial. Trials, 17, 154.

Boh, B., Jansen, A., Clijsters, I., Lemmens, L. H. J. M., Nederkoorn, C., Spanakis, G., \& Roefs, A. (2016). Indulgent thinking? Ecological Momentary Assessment of Overweight and Healthy-weight Participants' Cognitions and Emotions. Behaviour Research and Therapy, 87, 196-206.

Bongers, P., de Graaff, A., \& Jansen, A. (2016). "Emotional" does not even start to cover it: Generalization of overeating in emotional eaters. Appetite, 96, 611-616.

Bongers, P., Jansen, A., Havermans, R., Roefs, A., \& Nederkoorn, C. (2013). Happy eating: the underestimated role of overeating in a positive mood. Appetite, 67, 74-80.

Bongers, P., Jansen, A., Houben, K., \& Roefs, A. (2013). Happy eating: The single target implicit association test predicts overeating after positive emotions. Eating Behaviors, 14, 348-355.

Borsboom, D., \& Cramer, A. O. J. (2013). Network analysis: an integrative approach to the structure of psychopathology. Annual Review of Clinical Psychology, 9, 91-121.

Bradburn, N. M., Rips, L. J., \& Shevell, S. K. (1987). Answering Autobiographical Questions: The Impact of Memory and Inference on Surveys. Science, 236, 157-161.

Bray, G. A. (2004). Obesity is a chronic, relapsing neurochemical disease. International Journal of Obesity, 28, 34-38.

Bringmann, L. F., Vissers, N., Wichers, M., Geschwind, N., Kuppens, P., Peeters, F., ... Tuerlinckx, F. (2013). A network approach to psychopathology: new insights into clinical longitudinal data. Plos One, 8, e60188.

Brownell, K. D. (1991). The Heterogeneity of Obesity: Fitting Treatments To Individuals. Behavior Therapy, 22, 153-177.

Brownell, K. D. (2010). The humbling experience of treating obesity: Should we persist or desist? Behaviour Research and Therapy, 48, 717-9.

Butler, A., Chapman, J., Forman, E., \& Beck, A. (2006). The empirical status of cognitive-behavioral therapy: A review of meta-analyses. Clinical Psychology Review, 26, 17-31.

Butryn, M. L., Webb, V., \& Wadden, T. A. (2011). Behavioral Treatment of Obesity. Psychiatric Clinics of North America, 34, 841-859.

Burnett, K. F., Taylor, C. B., \& Agras, W. S. (1985). Ambulatory computer-assisted therapy for obesity: a new frontier for behavior therapy. Journal of Consulting and Clinical Psychology, 53, 698-703.

Burnett, K. F., Taylor, C. B., \& Agras, W. S. (1992). Ambulatory computer-assisted behavior therapy for obesity: An empirical model for examining behavioral correlates of treatment outcome. Computers in Human Behavior, 8, 239-248.

Byrne, S. M. (2002). Psychological aspects of weight maintenance and relapse in obesity. Journal of Psychosomatic Research, 53, 1029-36.

Cardi, V., Leppanen, J., \& Treasure, J. (2015). The effects of negative and positive mood induction on eating behaviour: a meta-analysis of laboratory studies in the healthy population and eating and weight disorders. Neuroscience \& Biobehavioral Reviews, 57, 299-309. 
Carels, R. A., Hoffman, J., Collins, A., Raber, A. C., Cacciapaglia, H., \& O'Brien, W. H. (2001). Ecological momentary assessment of temptation and lapse in dieting. Eating Behaviors, 2, 307-21.

Carels, R. A., Douglass, O. M., Cacciapaglia, H. M., \& O'Brien, W. H. (2004). An ecological momentary assessment of relapse crises in dieting. Journal of Consulting and Clinical Psychology, 72, 341-8.

Carter, F. A, \& Jansen, A. (2012). Improving psychological treatment for obesity. Which eating behaviours should we target? Appetite, 58, 1063-9.

Carter, R., Mouralidarane, A., Ray, S., Soeda, J., \& Oben, J. (2012). Recent advancements in drug treatment of obesity. Clinical Medicine, 12, 456-60.

Cawley, J., \& Meyerhoefer, C. (2012). The medical care costs of obesity: an instrumental variables approach. Journal of Health Economics, 31, 219-30.

Chao, A., Grilo, C. M., White, M. a., \& Sinha, R. (2014). Food cravings, food intake, and weight status in a community-based sample. Eating Behaviors, 15, 478-482.

Chang, S.-H., Stoll, C. R. T., Song, J., Varela, J. E., Eagon, C. J., \& Colditz, G. A. (2014). The effectiveness and risks of bariatric surgery. JAMA Surgery, 149, 275.

Chua, J. L., Touyz, S., \& Hill, A. J. (2004). Negative mood-induced overeating in obese binge eaters: an experimental study. International Journal of Obesity, 28, 606-610.

Cohen, E. A., Gelfand, D. M., Dodd, D. K., Jensen, J., \& Turner, C. (1980). Self-control practices associated with weight loss maintenance in children and adolescents. Behavior Therapy, 11, 26-37.

Cohen, J. (1992). Statistical Power Analysis. Current Directions in Psychological Science, 1, 98-101.

Cohen, D., \& Farley, T. A. (2008). Eating as an automatic behavior. Preventing Chronic Disease, 5, A23.

Cooper, M. J., \& Fairburn, C. G. (1992). Thoughts about eating, weight and shape in anorexia nervosa and bulimia nervosa. Behaviour Research and Therapy, 30, 501-511.

Cooper, Z., \& Fairburn, C. G. (2001). A new cognitive behavioural approach to the treatment of obesity. Behaviour Research and Therapy, 39, 499-511.

Cooper, Z., Doll, H. A., Hawker, D. M., Byrne, S., Bonner, G., Eeley, E., .. Fairburn, C. G. (2010). Testing a new cognitive behavioural treatment for obesity: A randomized controlled trial with three-year follow-up. Behaviour Research and Therapy, 48, 706-13.

Cruise, C. E., Broderick, J., Porter, L., Kaell, A., \& Stone, A. A. (1996). Reactive effects of diary self-assessment in chronic pain patients. Pain, 67, 253-8.

Curioni, C. C., \& Lourenço, P. M. (2005). Long-term weight loss after diet and exercise: a systematic review. International Journal of Obesity, 29, 1168-74.

Dansinger, M. L., Tatsioni, A., Wong, J. B., Chung, M., \& Balk, E. M. (2007). Meta-Analysis: The Effect of Dietary Counseling for Weight Loss. Annals of Internal Medicine, 147, 41-50.

Davis, C., Patte, K., Levitan, R., Reid, C., Tweed, S., \& Curtis, C. (2007). From motivation to behaviour: a model of reward sensitivity, overeating, and food preferences in the risk profile for obesity. Appetite, 48, 12-9.

Davis, C., Strachan, S., \& Berkson, M. (2004). Sensitivity to reward: Implications for overeating and overweight. Appetite, 42, 131-138.

Delahanty, L. M., Meigs, J. B., Hayden, D., Williamson, D. A., \& Nathan, D. M. (2002). Psychological and behavioral correlates of baseline BMI in the Diabetes Prevention Program (DPP). Diabetes Care, 25, 1992-1998.

Derogatis, L. D., Melisaratos, N. (1983). The Brief Symptom Inventory: An introductory report. Psychological Medicine, 13, 595-605.

Dixon, J. B. (2010). The effect of obesity on health outcomes. Molecular and Cellular Endocrinology, 316(2), 104-108.

Douketis, J. D., Macie, C., Thabane, L., \& Williamson, D. F. (2005). Systematic review of long-term weight loss studies in obese adults: clinical significance and applicability to clinical practice. International Journal of Obesity, 29, 1153-67. 
Eisenberg, D., Downs, M. F., Golberstein, E., \& Zivin, K. (2009). Stigma and Help Seeking College Students. Medical Care Research And Review, 66, 522-541.

Egger, G., \& Swinburn, B. (1997). An "Ecological" Approach to the Obesity Pandemic. British Medical Journal, $315,477-480$

Engel, S. G., Wonderlich, S. A., Crosy, R. D., Mitchell, J. E., Crow, S., Peterson, C. B., .. Gordon, K. H. (2013). The Role of affect in the maintenance of anorexia nervosa: evidence from a naturalistic assessment of momentary behaviors and emotion. Journal of Abnormal Psychology, 122, 709-719.

Epstein, L. H., Temple, J. L., Roemmich, J. N., \& Bouton, M. E. (2009). Habituation as a determinant of human food intake. Psychological Review, 116, 384-407.

Evers, C., Adriaanse, M., de Ridder, D. T. D., \& de Witt Huberts, J. C. (2013). Good Mood Food. Positive emotion as a neglected trigger for food intake. Appetite, 68, 1-7.

Evers, C., Marijn Stok, F., \& de Ridder, D. T. D. (2010). Feeding your feelings: emotion regulation strategies and emotional eating. Personality \& Social Psychology Bulletin, 36, 792-804.

Evers, C., de Ridder, D. T. D., \& Adriaanse, M. A. (2009). Assessing yourself as an emotional eater: mission impossible? Health Psychology, 28, 717-25.

Fairburn, C. G., \& Beglin, S. J. (1994). Assessment of eating disorders: interview or self-report questionnaire? International Journal of Eating Disorders, 16, 363-70.

Fairburn, C. G., Cooper, Z., \& Shafran, R. (2003). Cognitive behaviour therapy for eating disorders: a "transdiagnostic" theory and treatment. Behaviour Research and Therapy, 41, 509-528.

Faith, M. S., Fontaine, K. R., Baskin, M. L., \& Allison, D. B. (2007). Toward the reduction of population obesity: macrolevel environmental approaches to the problems of food, eating, and obesity. Psychological Bulletin, 133, 205-26.

Faul, F., Erdfelder, E., Lang, A. G., \& Buchner, A. (2007). G*Power 3: A flexible statistical power analysis program for the social, behavioral, and biomedical sciences. Behavior Research Methods, 39, 175-191.

Flegal, K. M., Carroll, M. D., Kit, B. K., \& Ogden, C. L. (2012). Prevalence of obesity and trends in the distribution of body mass index among US adults, 1999-2010. Journal of the American Medical Association, 307, 491-7.

Flegal, K. M., Carroll, M. D., Ogden, C. L., \& Curtin, L. R. (2010). Prevalence and trends in obesity among US adults, 1999-2008. Journal of the American Medical Association, 303, 235-41.

Foreyt, J. P., Carlos, W. S., Poston, I., \& John, P. (1998). What Is the Role of Cognitive-Behavior Therapy in Patient Management? Obesity Research, 6 Suppl 1, 18S-22S.

Foroushani, P., Schneider, J., \& Assareh, N. (2011). Meta-review of the effectiveness of computerised CBT in treating depression. BMC Psychiatry, 11, 131.

Franken, I. H. A., \& Muris, P. (2005). Individual differences in reward sensitivity are related to food craving and relative body weight in healthy women. Appetite, 45, 198-201.

Fredrickson, B. L. (2000). Extracting meaning from past affective experiences: The importance of peaks, ends , and specific emotions. Cognition and Emotion, 14, 577-607.

Franz, M. J., VanWormer, J. J., Crain, A. L., Boucher, J. L., Histon, T., Caplan, W., ... Pronk, N. P. (2007). Weight-loss outcomes: a systematic review and meta-analysis of weight-loss clinical trials with a minimum 1-year follow-up. Journal of the American Dietetic Association, 107, 1755-67.

Franz, M. J., Boucher, J. L., Rutten-Ramos, S., \& VanWormer, J. J. (2015). Lifestyle Weight-Loss Intervention Outcomes in Overweight and Obese Adults with Type 2 Diabetes: A Systematic Review and MetaAnalysis of Randomized Clinical Trials. Journal of the Academy of Nutrition and Dietetics, 115, 1447-1463

Fujioka, K. (2002). Management of obesity as a chronic disease: nonpharmacologic, pharmacologic, and surgical options. Obesity Research, 10 Suppl 2, 116S-123S. 
Fuller-Tyszkiewicz, M., Skouteris, H., Richardson, B., Blore, J., Holmes, M., \& Mills, J. (2013). Does the burden of the experience sampling method undermine data quality in state body image research? Body Image, 10,607-613.

Gade, H., Hjelmesæth, J., Rosenvinge, J. H., \& Friborg, O. (2014). Effectiveness of a Cognitive Behavioral Therapy for Dysfunctional Eating among Patients Admitted for Bariatric Surgery: A Randomized Controlled Trial. Journal of Obesity, 2014, 1-6.

Gerhards, S. A. H., de Graaf, L. E., Jacobs, L. E., Severens, J. L., Huibers, M. J. H., Arntz, a, ... Evers, S. M. A. A. (2010). Economic evaluation of online computerised cognitive-behavioural therapy without support for depression in primary care: randomised trial. British Journal of Psychiatry, 196, 310-8.

de Graaf, L. E., Huibers, M. J. H., Riper, H., Gerhards, S. A. H., \& Arntz, A. (2009). Use and acceptability of unsupported online computerized cognitive behavioral therapy for depression and associations with clinical outcome. Journal of Affective Disorders, 116, 227-31.

de Ridder, D., Adriaanse, M., Evers, C., \& Verhoeven, A. (2014). Who diets? Most people and especially when they worry about food. Appetite, 80, 103-108.

de Zwaan, M. (2001). Binge eating disorder and obesity. International Journal of Obesity, 25, S51-S55.

Goldschmidt, A. B., Crosby, R. D., Cao, L., Engel, S. G., Durkin, N., Beach, H. M., .. Peterson, C. B. (2014). Ecological momentary assessment of eating episodes in obese adults. Psychosomatic Medicine, 76, 747-752.

Goldschmidt, A. B., Crosby, R. D., Engel, S. G., Crow, S. J., Cao, L., Peterson, C. B., \& Durkin, N. (2014). Affect and eating behavior in obese adults with and without elevated depression symptoms. International Journal of Eating Disorders, 47, 281-6.

Goldstein, D. J. (1992). Beneficial health effects of modest weight loss. International Journal of Obesity and Related Metabolic Disorders, 16, 397-415.

Grenard, J. L., Stacy, A. W., Shiffman, S., Baraldi, A. N., MacKinnon, D. P., Lockhart, G., ... Reynolds, K. D. (2013). Sweetened drink and snacking cues in adolescents: a study using ecological momentary assessment. Appetite, 67, 61-73.

Gudzune, K. A., Doshi, R. S., Mehta, A. K., Chaudhry, Z. W., Jacobs, D. K., Vakil, R. M., ... Clark, J. M. (2015). Efficacy of Commercial Weight-Loss Programs. Annals of Internal Medicine, 162, 501.

Haddock, C. K., Poston, W. S. C., Dill, P. L., Foreyt, J. P., \& Ericsson, M. (2002). Pharmacotherapy for obesity: a quantitative analysis of four decades of published randomized clinical trials. International Journal of Obesity and Related Metabolic Disorders, 26, 262-273.

Harvey-Berino, J., Pintauro, S., Buzzell, P., \& Gold, E. C. (2004). Effect of internet support on the long-term maintenance of weight loss. Obesity Research, 12, 320-9.

Hays, N. P., \& Roberts, S. B. (2008). Aspects of eating behaviors "disinhibition" and "restraint" are related to weight gain and BMl in women. Obesity, 16, 52-8.

Heerstrass, D. W., Ocké, M. C., Bueno-De-Mesquita, H. B., Peeters, P. H. M., \& Seidell, J. C. (1998). Underreporting of energy protein and potassium intake in relation to body mass index. International Journal of Epidemiology, 27, 186-193.

Hektner, J. M., Schmidt, J. A., \& Csikszentmihalyi, M. (2006). Experience sampling method: Measuring the quality of everyday life. Thousand Oaks, CA: Sage.

Herman, C. P., \& Mack, D. (1975). Restrained and unrestrained eating. Journal of Personality, 43, 647-60.

Heron, K. E., \& Smyth, J. M. (2013). Is intensive measurement of body image reactive? A two-study evaluation using Ecological Momentary Assessment suggests not. Body Image, 10, 35-44.

Hilbert, A., \& Tuschen-Caffier, B. (2007). Maintenance of Binge Eating through Negative Mood: A Naturalistic Comparison of Binge Eating Disorder and Bulimia Nervosa. International Journal of Eating Disorders, $40,521-530$.

Hill, J. O., \& Peters, J. C. (1998). Environmental Contributions to the Obesity Epidemic. Science, 280, 1371-1374. 
Hill, J. O., Wyatt, H., Phelan, S., \& Wing, R. (2005). The National Weight Control Registry: is it useful in helping deal with our obesity epidemic? Journal of Nutrition Education and Behavior, 37, 206-10.

Hill, A. J. (2007). The psychology of food craving. The Proceedings of the Nutrition Society, 66, 277-285.

Hofmann, W., Adriaanse, M., Vohs, K. D., \& Baumeister, R. F. (2013). Dieting and the self-control of eating in everyday environments: An experience sampling study. British Journal of Health Psychology, 1-17.

Hofmann, W., Friese, M., \& Roefs, A. (2009). Three ways to resist temptation: The independent contributions of executive attention, inhibitory control, and affect regulation to the impulse control of eating behavior. Journal of Experimental Social Psychology, 45, 431-435.

Hollon, S. D., \& Beck, A. T. (1994). Cognitive and cognitive-behavioural therapies. In A. E. Bergin, \& S. L. Garfield (Eds.), Handbook of psychotherapy and behavior change, (4th ed.), 428-466.

Holm, S. (1979). A Simple Sequentially Rejective Multiple Test Procedure. Scandinavian Journal of Statistics, 6, $65-70$.

Houben, K. (2011). Overcoming the urge to splurge: influencing eating behavior by manipulating inhibitory control. Journal of Behavior Therapy and Experimental Psychiatry, 42, 384-8.

Ilies, R., Dimotakis, N., \& Watson, D. (2010). Mood, blood pressure, and heart rate at work: an experiencesampling study. Journal of Occupational Health Psychology, 15, 120-130.

Ikeda, J., Amy, N. K., Ernsberger, P., Gaesser, G. A., Berg, F. M., Clark, C. A., .. Peters, P. (2005). The National Weight Control Registry: a critique. Journal of Nutrition Education and Behavior, 37, 203-5.

Jansen, A., Houben, K., \& Roefs, A. (2015). A Cognitive Profile of Obesity and Its Translation into New Interventions. Frontiers in Psychology, 6(Suppl.), 282.

Jansen, A., \& Mulkens, S. (2015). Leren om niet te eten. Een programma voor behandelaars van mensen met overgewicht. Maastricht: Mediview.

Jansen, A., Nederkoorn, C., Roefs, A., Bongers, P., Teugels, T., \& Havermans, R. (2011). The proof of the pudding is in the eating: is the DEBQ-external eating scale a valid measure of external eating? International Journal of Eating Disorders, 44, 164-8.

Jansen, A., Vanreyten, A., van Balveren, T., Roefs, A., Nederkoorn, C., \& Havermans, R. (2008). Negative affect and cue-induced overeating in non-eating disordered obesity. Appetite, 51, 556-62.

Jansen, E., Mulkens, S. \& Jansen, A. (2011). Tackling childhood overweight: treating parents exclusively is effective. Journal of Obesity, 35, 501 - 509.

Jeffery, R. W., Drewnowski, A., Stunkard, A. J., Epstein, L. H., Wilson, G. T., Wing, R. R., \& Hill, D. R. (2000). LongTerm Maintenance of Weight Loss: Current Status. Health Psychology, 19 (Suppl.), 5-16.

Jeffery, R. W., \& Levy, R. L. (2010). Overgeneralization from limited data: A commentary on Cooper et al., 2010. Behaviour Research and Therapy, 48, 714-6.

Jia, H., \& Lubetkin, E. I. (2005). The impact of obesity on health-related quality-of-life in the general adult US population. Journal of Public Health, 27, 156-164.

Johnston, B. C., Kanters, S., Bandayrel, K., Wu, P., Naji, F., Siemieniuk, R. A., ... Mills, E. J. (2014). Comparison of Weight Loss Among Named Diet Programs in Overweight and Obese Adults. Journal of the American Medical Association, 312, 923.

Jones, M., Luce, K. H., Osborne, M. I., Taylor, K., Cunning, D., Doyle, A. C., ... Taylor, C. B. (2008). Randomized, controlled trial of an internet-facilitated intervention for reducing binge eating and overweight in adolescents. Pediatrics, 121, 453-62.

Kaltenthaler, E., Sutcliffe, P., Parry, G., Beverley, C., Rees, A., \& Ferriter, M. (2008). The acceptability to patients of computerized cognitive behaviour therapy for depression: a systematic review. Psychological Medicine, 38, 1521-30. 
Karhunen, L., Lyly, M., Lapveteläinen, A., Kolehmainen, M., Laaksonen, D. E., Lähteenmäki, L., Poutanen, K. (2012). Psychobehavioural factors are more strongly associated with successful weight management than predetermined satiety effect or other characteristics of diet. Journal of Obesity, 2012.

Kasen, S., Cohen, P., Chen, H., \& Must, a. (2008). Obesity and psychopathology in women: a three decade prospective study. International Journal of Obesity, 32, 558-566.

Kenardy, J., Butler, A., Carter, C., \& Moor, S. (2003). Eating, mood, and gender in a noneating disorder population. Eating Behaviors, 4, 149-158.

Koball, A. M., Meers, M. R., Storfer-Isser, A., Domoff, S. E., \& Musher-Eizenman, D. R. (2012). Eating when bored: Revision of the Emotional Eating Scale with a focus on boredom. Health Psychology, 31, 521-524.

Kołakowska, A., Landowska, A., Szwoch, M., Szwoch, W., \& Wróbel, M. R. (2013). Emotion Recognition and its Application in Software Engineering. In 6th International Conference on Human System Interaction (pp. 532-539).

Kramer, I., Simons, C. J. P., Hartmann, J. A., Menne-Lothmann, C., Viechtbauer, W., Peeters, F., ... Wichers, M. (2014). A therapeutic application of the experience sampling method in the treatment of depression : a randomized controlled trial. World Psychiatry, 13, 68-77.

Kretsch, M. J., Fong, A. K. H., \& Green, M. W. (1999). Behavioral and body size correlates of energy intake underreporting by obese and normal-weight women. Journal of the American Dietetic Association, 99, 300-306.

Krukowski, R. A., Harvey-Berino, J., Ashikaga, T., Thomas, C. S., \& Micco, N. (2008). Internet-based weight control: the relationship between web features and weight loss. Telemedicine Journal and E-Health, 14, 775-82.

Kubiak, T., Vögele, C., Siering, M., Schiel, R., \& Weber, H. (2008). Daily hassles and emotional eating in obese adolescents under restricted dietary conditions--the role of ruminative thinking. Appetite, 51, 206-9.

Lang, A., \& Froelicher, E. S. (2006). Management of overweight and obesity in adults: behavioral intervention for long-term weight loss and maintenance. European Journal of Cardiovascular Nursing, 5, 102-14.

Larsen, J. K., van Strien, T., Eisinga, R., \& Engels, R. C. M. E. (2006). Gender differences in the association between alexithymia and emotional eating in obese individuals. Journal of Psychosomatic Research, 60, 237-243.

Leahey, T. M., Crowther, J. H., \& Ciesla, J. A. (2011). An Ecological Momentary Assessment of the Effects of Weight and Shape Social Comparisons on Women With Eating Pathology, High Body Dissatisfaction, and Low Body Dissatisfaction. Behavior Therapy, 42, 197-210.

Ledikwe, J. H., Ello-Martin, J. A., \& Rolls, B. J. (2005). Symposium : Modifying the Food Environment: Energy Density , Food Costs, and Portion Size Food Choices and Diet Costs: an Economic Analysis. The Journal of Nutrition, 900-904.

Lluch, A., Herbeth, B., Méjean, L., \& Siest, G. (2000). Dietary intakes, eating style and overweight in the Stanislas Family Study. International Journal of Obesity and Related Metabolic Disorders, 24, 1493-1499.

Lowe, M. R., \& Butryn, M. L. (2007). Hedonic hunger: a new dimension of appetite? Physiology \& Behavior, 91 , 432-9.

Loxton, N. J., Dawe, S., \& Cahill, A. (2011). Does negative mood drive the urge to eat? The contribution of negative mood, exposure to food cues and eating style. Appetite, 56, 368-374.

Macht, M., Haupt, C., \& Salewsky, A. (2004). Emotions and eating in everyday life: application of the experiencesampling method. Ecology of Food and Nutrition, 43, 11-21.

Martin, C. K., O'Neil, P. M., Tollefson, G., Greenway, F. L., \& White, M. A. (2008). The association between food cravings and consumption of specific foods in a laboratory taste test. Appetite, 51, 324-326.

Mehl, M. R., \& Conner, T. S. (Eds.). (2012). Handbook of Research Methods for Studying Daily Life. New York, NY: The Guildford Press. 
McCrory, M. A., Fuss, P. J., McCallum, J. E., Yao, M., Vinken, A. G., Hays, N. P., \& Roberts, S. B. (1999). Dietary variety within food groups: Association with energy intake and body fatness in men and women. American Journal of Clinical Nutrition, 69, 440-447.

McNally, R. J. (2001). On the scientific status of cognitive appraisal models of anxiety disorder. Behaviour Research and Therapy, 39, 513-521.

McKee, H. C., Ntoumanis, N., \& Taylor, I. M. (2014). An Ecological Momentary Assessment of Lapse Occurrences in Dieters. Annals of Behavioral Medicine.

McKinsey Global Institute. (2014). Overcoming obesity: An initial economic analysis.

Mertens, I. L., \& Van Gaal, L. F. (2000). Overweight, obesity, and blood pressure: the effects of modest weight reduction. Obesity Research, 8, 270-278.

Middleton, K. M. R., Patidar, S. M., \& Perri, M. G. (2012). The impact of extended care on the long-term maintenance of weight loss: a systematic review and meta-analysis. Obesity Reviews, 13, 509-17.

Mietus-Snyder, M. L., \& Lustig, R. H. (2008). Childhood obesity: adrift in the "limbic triangle". Annual Review of Medicine, 59, 147-62.

Moher, D., Hopewell, S., Schulz, K. F., Montori, V., Gotzsche, P. C., Deveraux, P. J., Elbourne, D., Egger, M., \& Altman, D. G. (2010). Research methods and reporting: CONSORT 2010 explanation and elaboration: updated guidelines for reporting parallel group randomised trials. British Medical Journal (Clinical Research Edition), 340, c869.

Moroshko, I., Brennan, L., \& O'Brien, P. (2011). Predictors of dropout in weight loss interventions: A systematic review of the literature. Obesity Reviews, 12, 912-934.

Mozaffarian, D., Hao, T., Rimm, E. B., Willett, W. C., Hu, F. B., \& Ph, D. (2011). Changes in Diet and Lifestyle and Long- Term Weight Gain in Women and Men. New England Journal of Medicine, 364, 2392-2404f.

Munsch, S., Michael, T., \& Meyer, H. (2008). Randomized Controlled Comparison of Two Cognitive Behavioral Therapies for Obese Children: Mother versus Mother-Child Cognitive Behavioral Therapy. Psychotherapy and Psychosomatics, 77, 235-246.

Nauta, H., Hospers, H. J., Jansen, A., \& Kok, G. (2000). Cognitions in Obese Binge Eaters and Obese Non-Binge Eaters. Cognitive Therapy and Research, 24, 521-531.

Neve, M., Morgan, P. J., Jones, P. R., \& Collins, C. E. (2010). Effectiveness of web-based interventions in achieving weight loss and weight loss maintenance in overweight and obese adults: a systematic review with meta-analysis. Obesity Reviews, 11, 306-21.

Newell, D. J. (1992). Intention-to-treat analysis: implications for quantitative and qualitative research. International Journal of Epidemiology, 21, 837-841.

Ng, M., Fleming, T., Robinson, M., Thomson, B., Graetz, N., Margono, C., ... Gakidou, E. (2014). Global, regional, and national prevalence of overweight and obesity in children and adults during 1980-2013: A systematic analysis for the Global Burden of Disease Study 2013. The Lancet, 384, 766-781.

Nour, M. M., Chen, J., \& Allman-Farinelli, M. (2015). Efficacy and External Validity of Electronic and Mobile Phone-Based Interventions Promoting Vegetable Intake in Young Adults: A Systematic Review Protocol. Journal of Medical Internet Research, 4, e92.

O'Connor, D. B., \& O'Connor, R. C. (2004). Perceived changes in food intake in response to stress: The role of conscientiousness. Stress and Health, 20, 279-291.

O'Connor, D. B., Jones, F., Conner, M., Mcmillan, B., \& Ferguson, E. (2008). Effects of Daily Hassles and Eating Style on Eating Behavior. Health Psychology, 27, 20-31.

O'Connor, J., \& Dowrick, P. W. (1987). Cognitions in normal weight, overweight, and previously overweight adults. Cognitive Therapy and Research, 11, 315-326. 
Ogden, L. G., Stroebele, N., Wyatt, H. R., Catenacci, V. A., Peters, J. C., Stuht, J., ... Hill, J. O. (2012). Cluster analysis of the national weight control registry to identify distinct subgroups maintaining successful weight loss. Obesity, 20, 2039-47.

Oliver, G., Wardle, J., \& Gibson, E. L. (2000). Stress and Food Choice: A Laboratory Study. Psychosomatic Medicine, 62, 853-865.

Ouwehand, C., \& Papies, E. K. (2010). Eat it or beat it. The differential effects of food temptations on overweight and normal-weight restrained eaters. Appetite, 55(1), 56-60.

Overholser, J. C. (2010). Psychotherapy According to the Socratic Method: Integrating Ancient Philosophy With Contemporary Cognitive Therapy. Journal of Cognitive Psychotherapy, 24, 354-363.

Papies, E. K., Stroebe, W., \& Aarts, H. (2009). Understanding dieting: A social cognitive analysis of hedonic processes in self-regulation. European Review of Social Psychology, 19, 339-383.

Partridge, S. R., McGeechan, K., Hebden, L., Balestracci, K., Wong, A. T., Denney-Wilson, E., .. Allman-Farinelli, M. (2015). Effectiveness of a mHealth Lifestyle Program With Telephone Support (TXT2BFiT) to Prevent Unhealthy Weight Gain in Young Adults: Randomized Controlled Trial. Journal of Medical Internet Research, 3, e66.

Patel, K. A., \& Schlundt, D. G. (2001). Impact of moods and social context on eating behavior. Appetite, 36, $111-8$.

Patrick, K., Raab, F., Adams, M. A., Dillon, L., Zabinski, M., Rock, C. L., ... Norman, G. J. (2009). A text messagebased intervention for weight loss: Randomized controlled trial. Journal of Medical Internet Research, $11,1-9$.

Pelchat, M. L. (2002). Of human bondage: food craving, obsession, compulsion, and addiction. Physiology \& Behavior, 76, 347-52.

Perri, M. G., Sears, S. F., \& Clark, J. E. (1993). Strategies for Improving Maintenance of Weight Loss: Toward a continuous care model of obesity. Diabetes Care, 16, 200-210.

Perri, M. C. (1998). The Maintenance of Treatment Effects in the Long-Term Management of Obesity. Clinical Psychology: Science and Practice, 5, 526-543.

Perri, M. G., Sears, S. F., \& Clark, J. E. (1993). Strategies for Improving Maintenance of Weight Loss: Toward a continuous care model of obesity. Diabetes Care, 16, 200-210.

Pretlow, R. A. (2011). Addiction to highly pleasurable food as a cause of the childhood obesity epidemic: a qualitative Internet study. Eating Disorders, 19, 295-307.

Puhl, R. M., \& Heuer, C. A. (2010). Obesity stigma: important considerations for public health. American Journal of Public Health, 100, 1019-28.

Puhl, R. M., \& Brownell, K. D. (2003). Psychosocial origins of obesity stigma: toward changing a powerful and pervasive bias. Obesity Reviews, 4, 213-27.

Ramanathan, N., Alquaddoomi, F., Falaki, H., George, D., Hsieh, C., Jenkins, J., ... Estrin, D. (2012). Ohmage: An open Mobile System for Activity and Experience Sampling. In 6th International Conference on Pervasive Computing Technologies for Healthcare (Pervasive Health) (pp. 203-204).

Rancourt, D., Leahey, T. M., Larose, J. G., \& Crowther, J. H. (2015). Effects of weight-focused social comparisons on diet and activity outcomes in overweight and obese young women. Obesity, 23, 85-89.

Raynor, H. A., Jeffery, R. W., Phelan, S., Hill, J. O., Wing, R. R., Hollie, A., \& Rena, R. (2005). Amount of food group variety consumed in the diet and long-term weight loss maintenance. Obesity Research, 13, 883-90.

Raynor, H. A., \& Epstein, L. H. (2001). Dietary variety, energy regulation, and obesity. Psychological Bulletin, 127, 325-341.

Rippe, J. M., Crossley, S., \& Ringer, R. (1998). Obesity as a chronic disease. Journal of the American Dietetic Association, 98 Suppl 2, S9-S15. 
Rosenheck, R. (2008). Fast food consumption and increased caloric intake: A systematic review of a trajectory towards weight gain and obesity risk. Obesity Reviews, 9, 535-547.

Rozin, P., Fischler, C., Imada, S., Sarubin, A., \& Wrzesniewski, A. (1999). Attitudes to Food and the Role of Food in Life in the U. S. A ., Japan, Flemish Belgium and France: Possible Implications for the Diet-Health Debate. Appetite, 33, 163-180.

Saelens, B. E., \& Epstein, L. H. (1996). Reinforcing value of food in obese and non-obese women. Appetite, 27, $41-50$.

Sacks, F. M., Bray, G. A., Carey, V. J., Smith, S. R., Ryan, D. H., Anton, S. D., ... Williamson, D. A. (2009). Comparison of weight-loss diets with different compositions of fat, protein and carbohydrates. New England Journal of Medicine, 360, 859-873.

Saperstein, S. L., Atkinson, N. L., \& Gold, R. S. (2007). The impact of Internet use for weight loss. Obesity Reviews, 8, 459-65.

Sbrocco, T., Nedegaard, R. C., Stone, J. M., \& Lewis, E. L. (1999). Behavioral choice treatment promotes continuing weight loss: preliminary results of a cognitive-behavioral decision-based treatment for obesity. Journal of Consulting and Clinical Psychology, 67, 260-6.

Schwarz, N. (2007). Retrospective and Concurrent Self-Reports: The Rationale for Real-Time Data Capture. In A. Stone, S. Schiffman, A. Atienza, \& L. Nebeling (Eds.), The Science of Real-time Data Capture (pp. 11-26). Oxford University Press: New York.

Shapiro, J. R., Koro, T., Doran, N., Thompson, S., Sallis, J. F., Calfas, K., \& Patrick, K. (2012). Text4Diet: A randomized controlled study using text messaging for weight loss behaviors. Preventive Medicine, 55, 412-417.

Shaw, K., O'Rourke, P., Del Mar, C., \& Kenardy, J. (2005). Psychological interventions for overweight or obesity. Cochrane Database of Systematic Reviews, Issue 2.

Sherrington, A., Newham, J. J., Bell, R., Adamson, A., McColl, E., \& Araujo-Soares, V. (2016). Systematic review and meta-analysis of internet-delivered interventions providing personalized feedback for weight loss in overweight and obese adults. Obesity Reviews, 2016.

Shiffman, S. (2014). Conceptualizing analyses of ecological momentary assessment data. Nicotine \& Tobacco Research, 16 Suppl 2, S76-87.

Shiffman, S., Stone, A. A., \& Hufford, M. R. (2008). Ecological Momentary Assessment. Annual Review of Clinical Psychology, 4, 1-32

Spanakis, G., Weiss, G., Boh, B., \& Roefs, A. (2016). Network Analysis of Ecological Momentary Assessment Data for Monitoring and Understanding Eating Behavior. In Smart Health (Vol. 9545, pp. 43-54).

Speakman, J. R., \& Mitchell, S. E. (2011). Caloric restriction. Molecular Aspects of Medicine, 32, 159-221.

Stahre, L., \& Hällström, T. (2005). A short-term cognitive group treatment program gives substantial weight reduction up to 18 months from the end of treatment. A randomized controlled trial. Eating and Weight Disorders, 10, 51-58.

Stahre, L., Tärnell, B., Håkanson, C.-E., \& Hällström, T. (2007). A randomized controlled trial of two weightreducing short-term group treatment programs for obesity with an 18-month follow-up. International Journal of Behavioral Medicine, 14, 48-55.

Stone, A. A., Broderick, J. E., Schwartz, J. E., Shiffman, S., Litcher-Kelly, L., \& Calvanese, P. (2003). Intensive momentary reporting of pain with an electronic diary: reactivity, compliance, and patient satisfaction. Pain, 104, 343-351.

Swinburn, B. A., Sacks, G., Hall, K. D., McPherson, K., Finegood, D. T., Moodie, M. L., \& Gortmaker, S. L. (2011). The global obesity pandemic: shaped by global drivers and local environments. Lancet, 378, 804-14.

Swinburn, B., Egger, G., \& Raza, F. (1999). Dissecting obesogenic environments: the development and application of a framework for identifying and prioritizing environmental interventions for obesity. Preventive Medicine, 29, 563-70. 
van Strien, T., Frijters, J. E. R., Bergers, G. P. A., \& Defares, P. B. (1986). The Dutch Eating Behavior Questionnaire (DEBQ) for Assessment of Restrained, Emotional, and External Eating Behavior. International Journal of Eating Disorders, 5, 295-315.

van Strien, T., Herman, C. P., Anschutz, D. J., Engels, R. C. M. E., \& de Weerth, C. (2012). Moderation of distressinduced eating by emotional eating scores. Appetite, 58, 277-84.

Tafarodi, R. W., \& Swann, W. B. (1995). Self-Liking and Self-Competence as Dimensions of Global Self-Esteem. Journal of Personality Assessment, 65, 322-342.

Tate, D. F., Jackvony, E. H., \& Wing, R. R. (2006). A randomized trial comparing human e-mail counseling, computer-automated tailored counseling, and no counseling in an Internet weight loss program. Archives of Internal Medicine, 166, 1620-5.

Tate, D. F., Wing, R. R., \& Winett, R. A. (2001). Using Internet technology to deliver a behavioral weight loss program. Journal of the American Medical Association, 285, 1172-7.

Tiggemann, M. (2000). Dieting and cognitive style: the role of current and past dieting behaviour and cognitions. Journal of Health Psychology, 5, 17-24.

Tomiyama, A. J., Mann, T., \& Comer, L. (2009). Triggers of eating in everyday life. Appetite, 52, 72-82.

Vanderlinden, J., Adriaensen, A., Vancampfort, D., Pieters, G., Probst, M., \& Vansteelandt, K. (2012). A cognitive- behavioral therapeutic program for patients with obesity and binge eating disorder: short- and long- term follow-up data of a prospective study. Behavior Modification, 36, 670-86.

Vandromme, H., Hermans, D., Spruyt, A., \&Eelen, P. (2007). Dutch translation of the Self-Liking/Self-Competence Scale - Revised: A confirmatory factor analysis of the two-factor structure. Personality and Individual Differences, 42, 157-167.

Vincent, N., Walsh, K., \& Lewycky, S. (2010). Sleep locus of control and computerized cognitive-behavioral therapy (cCBT). Behaviour Research and Therapy, 48, 779-83.

Vreugdenburg, L., Bryan, J., \& Kemps, E. (2003). The effect of self-initiated weight-loss dieting on working memory: the role of preoccupying cognitions. Appetite, 41, 291-300.

Wang, Y. C., McPherson, K., Marsh, T., Gortmaker, S. L., \& Brown, M. (2011). Health and economic burden of the projected obesity trends in the USA and the UK. Lancet, 378, 815-25.

Wansink, B., \& Cashman, M. (2007). Mindless eating. Environment and Behavior, 39, 106-123.

Watts, S., Mackenzie, A., Thomas, C., Griskaitis, A., Mewton, L., Williams, A., \& Andrews, G. (2013). CBT for depression: a pilot RCT comparing mobile phone vs. computer. BMC Psychiatry, 13, 49.

Weingarten, H. P., \& Elston, D. (1991). Food cravings in a college population. Appetite, 17, 167-175.

Weinstein, P. K. (2006). A review of weight loss programs delivered via the Internet. The Journal of Cardiovascular Nursing, 21, 251-8-60.

Weiss, E. C., Galuska, D. a, Khan, L. K., \& Serdula, M. K. (2006). Weight-control practices among U.S. adults, 2001-2002. American Journal of Preventive Medicine, 31, 18-24.

Werrij, M. Q. (2005). Weighty Thoughts: A cognitive approach to the treatment of obesity (doctoral dissertation). Maastricht University, Maastricht.

Werrij, M. Q., Jansen, A., Mulkens, S., Elgersma, H. J., Ament, A. J. H. A., \& Hospers, H. J. (2009). Adding cognitive therapy to dietetic treatment is associated with less relapse in obesity. Journal of Psychosomatic Research, 67, 315-24.

Werthmann, J., Renner, F., Roefs, A., Huibers, M. J. H., Plumanns, L., Krott, N., \& Jansen, A. (2014). Looking at food in sad mood: Do attention biases lead emotional eaters into overeating after a negative mood induction? Eating Behaviors, 15, 230-236.

van Wier, M. F., Ariëns, G. A. M., Dekkers, J. C., Hendriksen, I. J. M., Smid, T., \& van Mechelen, W. (2009). Phone and e-mail counselling are effective for weight management in an overweight working population: a randomized controlled trial. BMC Public Health, 9, 6. 
White, M. A., Whisenhunt, B. L., Williamson, D. A., Greenway, F. L., \& Netemeyer, R. G. (2002). Development and validation of the food-craving inventory. Obesity Research, 10, 107-114.

White, R. (2010). Drugs and nutrition: how side effects can influence nutritional intake. The Proceedings of the Nutrition Society, 69, 558-64.

Wichers, M. (2013). The dynamic nature of depression: a new micro-level perspective of mental disorder that meets current challenges. Psychological Medicine, 1-12.

Wing, R. R., \& Hill, J. O. (2001). Successful Weight Loss Maintenance. Annual Review of Nutrition, 21, 323-41.

Wing, R. R., \& Phelan, S. (2005). Long-term weight loss maintenance. The American Journal of Clinical Nutrition, $82,222 \mathrm{~S}-225 \mathrm{~S}$.

Woolford, S. J., Barr, K. L. C., Derry, H. A., Jepson, C. M., Clark, S. J., Strecher, V. J., \& Resnicow, K. (2011). OMG do not say LOL: obese adolescents' perspectives on the content of text messages to enhance weight loss efforts. Obesity, 19, 2382-7.

Woolford, S. J., Clark, S. J., Strecher, V. J., \& Resnicow, K. (2010). Tailored mobile phone text messages as an adjunct to obesity treatment for adolescents. Journal of Telemedicine and Telecare, 16, 458-61.

Wu, T., Gao, X., Chen, M., \& van Dam, R. M. (2009). Long-term effectiveness of diet-plus-exercise interventions vs. diet-only interventions for weight loss: a meta-analysis. Obesity Reviews, 10, 313-323. 
Acknowledgments 

Bedankt aan iedereen die heeft bijgedragen aan de totstandkoming van mijn proefschrift. Mijn naam prijkt weliswaar aan de voorzijde, maar zonder jullie was ik er nooit gekomen. Het waren vier jaren vol voldoening, stress, lange dagen (en nachten), fijne tijden en piekermomenten, maar bovenal vol leerzame ontwikkeling. Graag wil ik een aantal bijzondere personen persoonlijk bedanken.

Mijn promotores: Anita, Anne, Lotte (nu ook!), en Sjaan. Het nieuws dat ik vier jaar lang onderzoek mocht doen naar de psychologie van eetgedrag, en een 'hardware apparatus' mocht ontwikkelen om mensen te helpen met hun eetproblemen, was fantastisch. Bedankt voor dat vertrouwen. Het apparaat is er niet gekomen, maar in plaats daarvan is er nu een heel mooie app. Anne, je was simpelweg onmisbaar voor de voortgang van het project en mijn ontwikkeling. Zonder je eeuwige geduld (geen superlatief teveel) met mijn chaotische trekken zou dit proefschrift er niet zijn geweest - dan was ik nu nog bezig met het opzetten van oeverloze analyses, modellen, en argumenten. Het was lang niet altijd makkelijk, maar juist als het dat niet was kon ik op je rekenen om nieuwe wegen in te slaan (ik heb de XXL proefschrift deadline kalender nog steeds!). Centraal stond daarbij steeds je concrete, heldere blik op complexe vraagstukken. Een blik die regelmatig tot laat in de avond via het digitale pad mijn inbox bereikte! Ik wist altijd precies waar ik aan toe was: de kwaliteit van mijn output zette je centraal, en als ik een pluim kreeg, had ik hem echt verdiend. Je hebt me binnen het project veel creatieve vrijheid gegeven, waardoor we uiteindelijk op het uitdagende EMA/EMI pad beland zijn, en daarmee ook de andere projecten binnen HLS hebben geïnspireerd. Ik denk met veel plezier terug aan onze lange discussies over de app. Je bent gewoon de beste AIO-begeleider die er is! Lotte, ik kende je als kamergenoot en ken je nu als co-promotor! In eerste instantie werd je bij het project betrokken als CGT specialist, maar dat groeide al snel in de breedte uit tot een fijne samenwerking bij het opzetten van de EMI studie. Je ervaringen vanuit je eigen promotietraject kwamen daarbij heel handig van pas: met veel wijsheid en inzicht werden er planning gemaakt en besprekingen georganiseerd die vaak erg soepel verliepen. Ik heb er veel van geleerd, en bewonder je assertiviteit! Sjaan, je stond klaar voor mijn vragen (waar ze dan ook over gingen), en dankzij je scherpe blik op vaak ingewikkelde keuzes heb ik flink wat knopen kunnen doorhakken. Je hebt de honneurs waargenomen toen Anne naar Italië was (buiten het Skypen met Anne om!), waarvoor dank! Anita, ik vond het erg fijn om deel uit te maken van de eetgroep! De onderlinge sfeer was altijd heel open en betrokken. Onherroepelijk een reflectie van jou als professor. Al leek de materie soms oneindig complex, op de een of andere manier kon je er altijd een duidelijke rode draad doorheen trekken. Ik kon ook altijd rekenen op sterke inzichten bij het reviseren van mijn manuscripten, waardoor ze kwalitatief enorm verbeterden. Een beter voorbeeld kon ik me niet wensen!

My knowledge engineering colleagues: Jerry and Gerhard. Without your efforts, we would not have been able to create a self-learning snack detection system! Jerry, you joined the project about halfway through, but it felt like you were part of the team since the start. You 
somehow knew right away what the project required. I appreciated your relaxed attitude and enjoyed discussing the project developments during our lunch meetings. Even though we came from two completely separate fields, this never really seemed to obstruct our communication. Gerhard, thank you for your valued input on project developments! It was always reassuring to have someone of your expertise express keen interest in what we were doing.

De technici die betrokken waren bij mijn project: Vincent, Michiel, Charlie en Richard. Ik heb de samenwerking met jullie als erg prettig ervaren. Vincent, ook jij kwam later bij het project, en vanuit een heel andere achtergrond. Zonder je hulp was de app er nooit gekomen - bedankt voor het vele programmeerwerk dat je hebt verricht. En bovenal bedankt voor het kunnen omgaan met de veranderlijkheid van de wetenschappelijke mening: soms moesten bepaalde functies, nadat je ze had geïmplementeerd, ineens heel anders. Dat gebeurde altijd zonder kleerscheuren! Vooral tijdens de hectische laatste weken voordat we van start gingen met de interventie studie, heb je bergen verzet om te zorgen dat alles voor de kerst af was, zelfs buiten kantooruren om! Ook tijdens de eerste weken van de studie, waarin er veel bugfixes verricht moesten worden, heb je zelfs in je eigen tijd gezorgd dat alles op rolletjes liep. Uiteindelijk was je veel meer dan een wetenschappelijk programmeur, en had je steeds inhoudelijk sterke input bij het opzetten van de studie. Maar, bovenal bedankt voor de prettige werksfeer! Michiel en Charlie, bedankt voor al het werk dat jullie samen met Lotte hebben verzet om te zorgen dat de web omgeving van de interventie op rolletjes liep. Het zag er allemaal uitstekend uit! Ook jullie bedankt voor jullie bereidheid om zelf buiten kantooruren nog wat last-minute aanpassingen door te voeren! Richard, je talent als (digitale) kunstenaar en je creatieve brein zijn niet te ontkennen. Zonder je input had de app er nooit zo mooi uitgezien als nu. Bedankt daarvoor!

De Philips onderzoekers die bij het project betrokken waren: Reinder en Jettie. Bedankt dat we veel vrijheid hebben gekregen om van het originele plan om een 'device / wearable' te ontwikkelen af te wijken! Uiteindelijk heeft dat gelukkig erg positief uitgepakt. De samenwerking met Philips was uitstekend. Reinder, als coördinator van HLS heb je vaak met ons om de tafel gezeten tijdens besprekingen. Onder het genot van een kop koffie of een lunch hebben we het gehad over de voortgang van het project. Je oprechte interesse en positieve houding heb ik altijd erg gewaardeerd. Jettie, als onze eigen Philips onderzoeker konden we altijd rekenen op je waardevolle input. Bedankt!

De eetgroep: Anita, Anne, Astrid, Bart, Carolien, Clare, Eric, Eva, Fania, Ghislaine, Haris, Iris, Janneke, Jessica A., Jessica W., Karolien, Katrijn, Lotte, Nele, Peggy, Remco, Sandra, Shannon, Sieske, Sjaan, en Valerie. Het was altijd erg gezellig in de eetgroep! Ik kon goed met iedereen overweg, en heb veel fijne contacten opgebouwd. Bedankt voor jullie inzichten tijdens onze wekelijkse 'eat meets.' Fania, Iris, Karolien en Sieske, samen zijn we uit menig kamer ontsnapt. Dat er nog vele mogen volgen! Jessica A., bedankt voor de gezellige bordspel 
avonden en 'potlucks', en voor de warme, openhartige gesprekken! Peggy, met amigurumi is het hier niets geworden, maar ach! Bedankt voor alle gezelligheid!

Mijn kamergenoten: eerst Andrea en Jantine, later Fritz en Lotte, en uiteindelijk Thomas F. en Jane. Bedankt voor de goede werksfeer, goede gesprekken, en koffiepauzes! Thomas F., we got along right away. I greatly appreciated your modest and welcoming personality and enjoyed our many talks about life, science and philosophy. Thank you and Anna for inviting me into your home to play Pandemic: Legacy! The legacy will continue, and I am sure we will conquer the pandemic! I wish you and Anna all the best with your son Bastian (great name, by the way!).

Mijn paranimfen: Kai en Thomas V. Boardgame fanaten pur sang, die me op aanstekelijke wijze hebben geleerd dat de door de fabrikant geschatte speltijd op de doos vaak toch echt moeten worden verdubbeld. Bedankt dat jullie mijn paranimfen wilden zijn (ondanks dat je speciaal hier heen zou moeten vliegen, Kai!), maar bovenal bedankt voor de vele te gekke avonden dat we tot laat strijd geleverd hebben op het karton. Ik heb veel zin in de volgende!

De studenten die betrokken waren bij het project, en me uitstekend geholpen hebben: Anastacia, Dorien, Ineke, en Lianne. Het was vaak hectisch, maar ik kon altijd op jullie rekenen - zelfs als er last-minute iemand moest invallen!

De administratieve helden van de derde verdieping: Jessie, Marionne, Caroline, Truus, en Lindy. Dankzij jullie gestructureerde en overzichtelijke aanpak heb ik de nodige administratieve misstap kunnen vermijden! Het was altijd gezellig op het secretariaat. Bedankt voor alle tips en handreikingen!

Marieke en Wolfgang, bedankt voor de interessante en nuttige inhoudelijke discussies over EMA / EMI, data analyse en studie design. Ik heb er erg veel aan gehad! Jullie open en pro-actieve houding ten opzichte van wetenschap heeft me erg geïnspireerd.

De andere boardgame fanaten met wie ik regelmatig strijd geleverd hebt: Alfons, Rob, Thomas F., en Tom. Bedankt voor de broodnodige afleiding!

Pim, ik deel je passie voor muziek en gitaren! Je had praktisch wekelijks weer een nieuwe gitaar, en dat was goede gespreksstof. Onze jamsessies vormden een fijne muzikale afleiding!

Nynke, bedankt voor de te gekke omslag van mijn proefschrift!

Carach Angren (Clemens, Dennis en Ivo) en Mescaliner (Lowie, Ludo en Rob). Ik hoop nog vele jaren met jullie te mogen musiceren. Bedankt voor alle mooie ervaringen, maar vooral bedankt voor het geduld en begrip dat jullie hebben gehad voor mijn drukke bestaan als promovendus.

Pap, mam, en Vincent. Bedankt dat ik altijd op jullie kan rekenen. 

Curriculum Vitae 

Bastiaan Boh was born on March 6, 1985, in Brunssum, the Netherlands. In July 2004 he graduated from secondary school (Atheneum, St. Janscollege, Hoensbroek). Following that, he studied psychology at Maastricht University, obtaining his BSc (Biological Psychology) in 2007, and his MSc (Cognitive Neuroscience research master track) in 2011. The research for his master's thesis was conducted at the Institut für Biomagnetismus und Biosignalanalyse at Münster University in Germany. In 2013, he started his PhD project at the Faculty of Psychology and Neuroscience at Maastricht University. He is currently working as a musician.

\section{PUBLICATIONS}

Boh, B., Jansen, A., Lemmens, L. H. J. M., Nederkoorn, C., Spanakis, G., \& Roefs, A. (In preparation). Randomized Controlled Trial of a Cognitive Behavioural Therapy-based Ecological Momentary Intervention for Obesity.

Boh, B., Jansen, A., Nederkoorn, C., Spanakis, G., \& Roefs, A. (In revision). Food Desire in Daily Life: Comparison of overweight and healthy-weight participants via ecological momentary assessment. Journal of Human Nutrition and Dietetics.

Boh, B., Jansen, A., Clijsters, I., Lemmens, L. H. J. M., Nederkoorn, C., Spanakis, G., \& Roefs, A. (2016). Indulgent thinking? Ecological Momentary Assessment of Overweight and Healthyweight Participants' Cognitions and Emotions. Behaviour Research and Therapy, 87, 196-206.

Boh, B., Lemmens, L. H. J. M., Jansen, A., Nederkoorn, C., Kerkhofs, V., Spanakis, G., ... Roefs, A. (2016). An Ecological Momentary Intervention for weight loss and healthy eating via smartphone and Internet: study protocol for a randomised controlled trial. Trials, 17, 154.

Spanakis, G., Weiss, G., Boh, B., Lemmens, L., \& Roefs, A. (2017). Machine learning techniques in eating behavior e-coaching. Personal and Ubiquitous Computing.

Spanakis, G., Weiss, G., Boh, B., Kerkhofs, V., \& Roefs, A. (2016). Utilizing Longitudinal Data to Build Decision Trees for Profile Building and Predicting Eating Behavior. In Procedia Computer Science (Vol 100, pp. 782-789).

Spanakis, G., Weiss, G., Boh, B., \& Roefs, A. (2015). Network analysis of Ecological Momentary Assessment data for monitoring and understanding eating behaviour. In Smart Health (Vol. 9545, pp. 43-54).

Boh, B., Herholz, S.C., Lappe, C., \& Pantev, C. (2011). Processing of Complex Auditory Patterns in Musicians and Nonmusicians. PloS One, 6, e21458.

Herholz, S.C., Boh, B., Pantev, C. (2011). Musical training modulates encoding of higherorder regularities in the auditory cortex. European Journal of Neuroscience, 34, 524-529. 


\section{CONFERENCE PRESENTATIONS AND DEMONSTRATIONS}

Boh, B. (2016). Think Slim. Demonstration given at the Vereniging voor Gedragstherapie en Cognitive Therapie (VGCt) najaarscongres. Veldhoven, The Netherlands.

Boh, B. (2016). Eating behaviour in daily life: From ecological momentary assessment to intervention. Presentation given at the Annual Experimental Psychopathology Candidate Day. Utrecht, The Netherlands.

Boh, B., Jansen, A., Spanakis, G., Kerkhofs, V., Lemmens, L., Weiss, G., \& Roefs, A. (2015). Eating behaviour in daily life: ecological momentary assessment of overweight and healthy-weight participants. Poster presented at the Healthy Lifestyle Solutions Conference. Eindhoven, The Netherlands.

Boh, B., Roefs, A., Nederkoorn, C., Lemmens, L., Spanakis, G., Kerkhofs, V., Weiss, G., \& Jansen, A. (2015). Train je brein, denk slank! Presentation given at the Vereniging voor Gedragstherapie en Cognitive Therapie (VGCt) najaarscongres. Veldhoven, The Netherlands.

Boh, B., Roefs, A., Clijsters, I., Claes, D., \& Jansen, A. (2014). Overeating in daily life. An ecological momentary assessment study. Poster presented at The 38th Annual Meeting of the British Feeding \& Drinking Group (BFDG).Portsmouth, United Kingdom.

Boh, B., Roefs, A., Nederkoorn, C., \& Jansen, A. (2013). Train je brein, denk slank! Geautomatiseerd interveniëren bij overgewicht en obesitas. Presentation given at the Vereniging voor Gedragstherapie en Cognitive Therapie (VGCt) najaarscongres. Veldhoven, The Netherlands. Boh, B., Roefs, A., Claes, D., Fossel, J., \& Jansen, A. (2013). Train your Brain, Think Slim! Mapping eating-related variables via a self-monitoring and experience sampling application. Poster presented and demonstration provided at the STW Annual Congress. Utrecht, The Netherlands.

Boh, B., Roefs, A., Claes, D., Fossel, J., \& Jansen, A. (2013). Train your Brain, Think Slim! Mapping eating-related variables via a self-monitoring and experience sampling application. Poster presented at the 3rd Conference of the Society for Ambulatory Assessment (SAA). Amsterdam, The Netherlands.

Boh, B., Roefs, A., Claes, D., Fossel, J., \& Jansen, A. (2013). Train your Brain, Think Slim! Mapping eating-related variables via a self-monitoring and experience sampling application. Poster presented at The 37th Annual Meeting of the British Feeding \& Drinking Group (BFDG). Loughborough, United Kingdom.

Herholz, S., Boh, B., Lappe, C. and Pantev, C. (2010). Neuronal correlates of complex pattern encoding within human auditory cortex are modulated by musical training. Poster presented at Biomag 2010-17th International Conference on Biomagnetism. Dubrovnik, Croatia. 

